\title{
Emerging Potential of Naturally Occurring Molecules against Gastric Cancer: Insights Molecular Mechanism and Therapeutic Targets
}

Review

Seog Young Kang 1, Dongwon Hwang 1, Soyoung Shin 1, Jinju Park 1, Myoungchan Kim 2, MD. Hasanur Rahman³, Md. Ataur Rahman ${ }^{1,2,4}$, Seong-Gyu Ko ${ }^{4}$ and Bonglee Kim 1, 2, 4, *

\author{
${ }^{1}$ College of Korean Medicine, Kyung Hee University, Hoegidong Dongdaemungu, Seoul 05253, Republic of \\ Korea. \\ 2Department of Pathology, College of Korean Medicine, Kyung Hee University, Hoegidong Dongdaemungu, \\ Seoul 05253, Republic of Korea. \\ ${ }^{3}$ Department of Biotechnology and Genetic Engineering, Bangabandhu Sheikh Mujibur Rahman Science and \\ Technology University, Gopalganj-8100, Bangladesh. \\ ${ }^{4}$ Korean Medicine-Based Drug Repositioning Cancer Research Center, College of Korean Medicine, Kyung Hee \\ University, Hoegidong Dongdaemungu, Seoul 05253, Republic of Korea. \\ * Correspondence: Bonglee Kim, Email: bongleekim@khu.ac.kr
}

\begin{abstract}
Gastric cancer, also known as stomach cancer, is a cancer which develops from the lining of the stomach. Accumulated evidences and epidemiological studies have been indicated that natural products play an important role in gastric cancer prevention and treatment, although its mechanism of action did not elucidate yet. Particularly, experimental studies have been showed that natural products displayed a protective effect against gastric cancer via numerous molecular mechanisms such as suppression of cell metastasis, anti-angiogenesis, inhibition of cell proliferation, induction of apoptosis, and modulation of autophagy. Although chemotherapy remains the standard treatment for advanced gastric cancer along with surgery, radiation therapy, hormone therapy and immunotherapy, but its adverse side effects including neutropenia, stomatitis, mucositis, diarrhea, nausea, and emesis are well documented. Additionally, intake of naturally occurring phytochemicals could increase the efficacy of gastric chemotherapy and chemotherapeutics resistance. However, natural product structural stability and powerful bioactivity are important to develop novel treatments for gastric cancer that may minimize such adverse effects. Therefore, the purpose of this review is to summarize the potential therapeutic effects of natural products on prevention and treatment of gastric cancer with intensive molecular mechanisms of action, bioavailability, and safety efficacy.
\end{abstract}

Keywords: Gastric cancer; natural products; autophagy; apoptosis; angiogenesis; metastasis; chemo-resistance

\section{Introduction}

The incidence and mortality of cancer is growing worldwide, with estimated 19.3 million new cases and 10 million cancer deaths in 2020 [1]. Gastric cancer is the $5^{\text {th }}$ most common neoplasm and the $4^{\text {th }}$ leading cause of cancer death, which led to over one million new cases and an estimated 769,000 deaths in 2020 [1]. Clinically, to offer pertinent treatment, gastric carcinoma is classified as early or advanced stage [2]. Gastric carcinoma has multiple risk factors; genetics, Helicobacter pylori infection, gastric ulcer, gastroesophageal reflux disease (GERD), tobacco, smoking, alcohol, chemical exposure, diet, obesity, and so forth $[3,4]$. Surgical resection, when possible, offers the best chances of cure for early gastric cancer [5]. Adjuvant or neoadjuvant chemotherapy may be beneficial in increasing the chance of successful resection or in decreasing the rate of recurrence and/or metastasis [68]. For patients with unresectable advanced gastric cancer, chemotherapy is a common 
choice. Conventional regimens are mostly based on cytotoxic agents including antimetabolites and platinum based anticancer drugs. However, these regimens cause severe side effects such as chemotherapy-induced peripheral neuropathy (CIPN), neutropenia, stomatitis, mucositis, diarrhea, nausea and emesis $[9,10]$. Moreover, failure of first-line chemotherapy due to resistance is also an obstacle of gastric cancer treatment hampering the novel and effective therapies and imposing significant economic costs to patients [11]. Exposure to unremovable toxins, trauma, or infection can occur mutagenic chronic inflammatory response, leading to dyplasia [12]. Considering gastric cancer, Helicobactor pylori infection is a major risk factor of developing deleterious tumor microenvironments [13]. Nuclear factor kappa-B (NF-kB), c-Jun N-terminal kinase (JNK), and signal transducer activator of transcription 3 (STAT3), inflammatory cytokines, tumor necrosis factor (TNF), interleukin (IL)-1/6, tumor-derived cytokines such as fasciclin (Fas) ligand and vascular endothelial growth factor (VEGF) are major targets of regulation for prevention and treatment of gastric cancer [14-17]. Therefore, novel drug development against gastric cancer is strongly needed to further improve survival rates of this disease and lower the side effect of conventional therapies.

Epidemiological studies showed that natural dietary products decrease the risks of gastric cancer [18-21]. Extensive research was conducted to measure the value of natural products for prevention and treatment of gastric carcinoma, leading to the discovery of major phytochemicals with anti-cancer properties such as quercetin, silymarin, taurine, berberine, curcumin, and so forth [22-25]. However, few review articles included agents from animal or marine sources, which are also being studied with growing expectation $[26,27]$. The same goes for traditional medicine, despite their wide use in clinical practice to combat various illnesses including cancer [28-31]. Especially, traditional Korean and Chinese medicine have used mixtures made from medicinal herbs, animals, or mineral resources. This review explored various single compounds or extracts isolated from biological resources, and traditional medicine in the form of mixtures that show anti-cancer properties, closely targeting gastric cancer. Efficacies and underlying mechanisms of the subjects are discussed as well as the major experimental models of each study.

\section{Role of naturally occurring molecules in gastric cancer managements}

Programmed cell death is an important process of cell suicide involved in a number of biological activities including morphogenesis, maintenance of tissue homeostasis, and harmful cell removal [32]. Since dysfunctional programmed cell death leads to several diseases, especially cancer, considering cell death is crucial for cancer treatment [32]. Various natural products are reported to suppress cancerous cell development by inducing cell death, apoptosis and autophagy [24]. Recent studies discovered that natural products can also trigger non-canonical cell death such as ferroptosis, necroptosis, and pyroptosis [33]. Among the studies discussed, one reported the occurrence of necrosis and another lipid peroxidation [34,35]. Other studies mainly reported induction of apoptosis and/or autophagy. Due to their wide variety, apoptosis-inducing natural products were organized into three subgroups according to their modes of preparation: single compounds, extracts, or mixtures (decoctions).

\subsubsection{Apoptosis-inducing natural molecules in gastric cancer}

Apoptosis is the process of programmed cell death, characterized by distinct morphology: cell shrinking, membrane blebbing, chromatin condensation, and nuclear fragmentation [36, 37]. Several single compounds and extracts originated from plants, animals, fungi, and minerals showed apoptosis-inducing effects on gastric cancer cells and animal models have been presented in Figure 1 and Table 1. Yang et al. reported that berberine could inhibit the proliferation of SGC-7901 cells and induce apoptosis on a dose dependent manner [38]. In vitro models have been demonstrated that cyclovirobuxine D originated from Buxux microphylla Richardii. Radix (Buxaceae) induced apoptosis in MGC803 and MKN-28 cells [39]. Both cell lines were treated 30, 60, $120 \mu \mathrm{M} / \mathrm{L}$ cyclovirobuxine D solutions for $48 \mathrm{~h}$. GFG-3a, a glycoprotein isolated from the fungus Grifola frondose 
(Diks.) Gray Mycelia (Meripilaceae), promoted apoptosis against SGC-7901 cells [40]. GFG$3 \mathrm{a}$ increased the expression levels of active caspases, p53 and Bcl-2 associated agonist of cell death (Bad). Kong et al. reported that melittin, a component of Apis cerena Fabricius venom (Apidae), induced caspase-dependent apoptosis by causing ROS formation and triggering mitochondrial membrane depolarization [41]. Expressions of caspase-3, cytochrome c, endonuclease G (Endo G), apoptosis inducing factor (AIF) and Smac/Diablo were upregulated in melittin-treated SGC-7901 cells. Wu et al. revealed that phenolic alkaloids of Menispermum dauricum DC. Rhizoma (Menispermaceae) induced apoptosis and suppressed gastric tumor growth by inducing apoptosis and inhibiting of oncogenic Kirsten rat sarcoma viral oncogene homolog (K-RAS) expression [42]. According to study by Zhang et al., berberine and d-Limonene showed anti-cancer effect synergistically [43]. Berberine was extracted from Coptidis japonica Makino rhizoma (Ranunculaceae), and d-limonene from Evodiae rutaecarpa Bentham. Fructus (Rutaceae). They were combined at a ratio of 1:4, 20 and $80 \mu \mathrm{M}$ each. Apoptotic rate of the combination of the two compounds was much higher (29.03\%) compared to berberine-only-treated group (12.2\%) and $d$-limoneneonly-treated group (19.6\%). Trifolirhizin, a compound isolated from Sophora flavescens Aiton Radix (Fabaceae), demonstrated apoptotic activity both in vitro and in vivo [44]. Trifolirhizin induced apoptosis of MKN-45 cells in vitro via EGFR-MAPK pathways and triggered G2/M phase cell cycle arrest by impacting CDC2/Cyclin B complex. Qian et al. discovered that Ginsenoside-Rh2 originated from Panax ginseng C.A.Mey Radix (Araliaceae) inhibits proliferation and induces apoptosis of SGC-7901 cells [45]. The ratio of Bcl-like protein 4 (Bax) to Bcl-2 (Bax/Bcl-2) was elevated following Ginsenoside-Rh2 treatment.

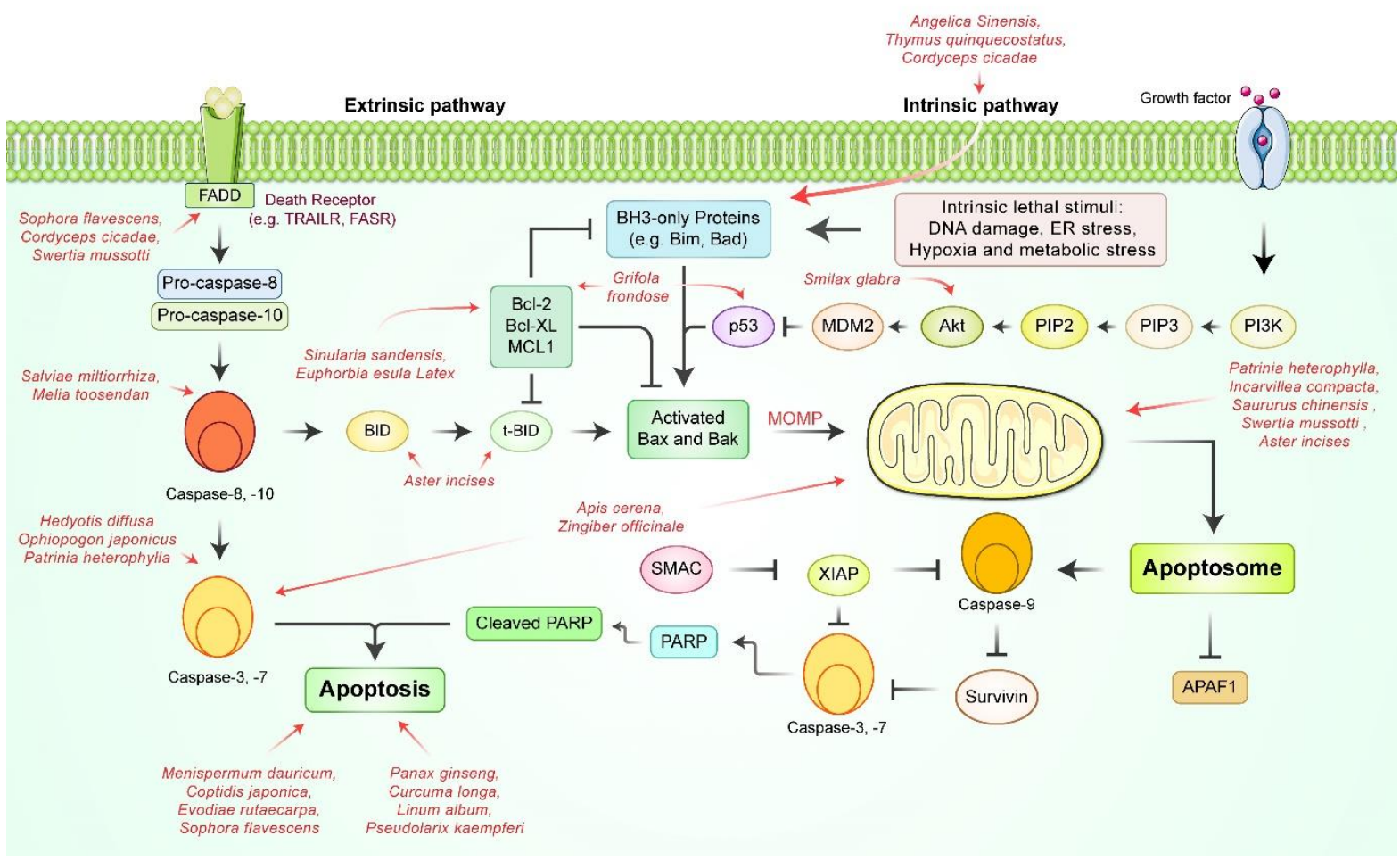

Figure 1. Schematic diagram of natural product-mediated apoptosis signaling pathways. FADD, Fas-associated proteins with death domain; TRAILR, TNF-related apoptosis-including ligand receptor; FASR, Fas receptor; tBid, truncated Bid; PARP, Poly ADP-ribose polymerase; APAF1, Apoptotic Protase Activating Factor 1; MOMP, Mitochondrial Outer Membrane Permeabilization; PIP2, Phosphatidylinositol-3,4-Bisphosphate; PIP3, Phosphatidylinositol-3,4,5-Triphosphate; PI3K, Phosphoinositide 3-kinase.

Tanshinone IIA, originated from Salviae miltiorrhiza Bunge. Radix (Lamiaceae), suppressed AGS gastric tumor cells via activation of tumor necrosis factor-alpha (TNF- $\alpha$ ), Fas, p38, JNK, p53, p21, caspase-3, caspase-8 and inhibition of ERK [46]. The apoptotic activities of Zingiber officinale Roscoe Rhizoma (Zingiberaceae) against AGS cells were reported by Debjani et al. [47]. 100, $250 \mu \mathrm{M}$ of [6]-gingerol treatment for $24 \mathrm{~h}$ to AGS cells 
generated reactive oxygen species and decreased $\Delta \Psi \mathrm{m}$, leading to induction of apoptosis. Perturbations of $\Delta \Psi \mathrm{m}$ were associated with deregulation of Bax/Bcl-2 ratio at protein level, which led to upregulation of cytochrome $c$ and triggered the caspase cascade. 2, 7dihydroxy-3-methylanthraquinone (DDMN), a flavone isolated from Hedyotis diffusa Willd. Herba, induced caspase-dependent apoptosis of SGC-7901 gastric cancer cells [48]. 6, 7, 30-trimethoxy-3, 5, 40-trihydroxy flavone (TTF), extracted from Chrysosplenium nudicaule Ledeb. Herba, is a well known traditional Chinese medicine for digestive diseases [49]. TTF induced apoptosis on SGC-7901 cells treated at the dose of 2, 4, 8, 16, $32 \mu \mathrm{g} / \mathrm{mL}$ for 24, 48 and $72 \mathrm{~h}$. Sun et al. observed that curcumin, isolated from Curcuma longa L. Rhizoma (Zingiberaceae), induced apoptosis of SGC-7901 and BGC-823 (5, 10, 15, 20, $40 \mu \mathrm{M}$ for $24 \mathrm{~h}$ ) cells by up-regulating microRNA-33b (miR-33b) expression [50]. Three human gastric cancer cell lines, SGC-7901, MGC-803, and BGC-823 were treated esculetin at dose of 12.5, 25, $50 \mu \mathrm{M}$ for $24 \mathrm{~h}$. Esculetin treatment triggered ROS formation, elevated caspase$3 / 9$ activity and induced poly (ADP-ribose) polymerases (PARP) cleavage. Liu et al. reported that hydroxysafflor yellow A (HSYA) induces apoptosis of BGC-7901 cells via activation of peroxisome proliferator-activated receptor gamma (PPAR $\gamma$ ) signal [51]. Expressions of PPAR $\gamma$ and caspase-3 were elevated. Zhou et al. studied the efficacy of the combination of TRAIL and kurarinone against gastric cancer cell line SGC-7901 [52]. Kurarinone is a substance originated from Sophora flavescens Aiton Radix (Fabaceae). Kurarinone $(5 \mu \mathrm{M})$ for $24 \mathrm{~h}$ significantly enhanced TRAIL-induced apoptosis compared to single treatment by stimulating inhibition of Mcl-1 expressions, cleavage of FLIP, and phosphorylation of STAT3. Wu et al. elucidated that licochalcone A (LicA) induced apoptosis by blocking the Akt signaling pathway and reducing hexokinase 2 (HK2) expression in MKN45 cells [53]. Notably, 25\% of tumor cells were subjected to apoptosis at $60 \mu \mathrm{M}$. The apoptotic ability of ophiopogonin B, a compound isolated from Ophiopogon japonicus Radix, against SGC-7901 cells were suspected to be relevant with the JNK 1/2 and ERK1/2 signaling pathways [54]. Ophiopogonin B treatment activated phosphorylation of JNK1/2 and ERK 1/2, upregulated active caspase-3 and Bax expression levels and downregulated $\mathrm{Bcl}-2$ expression levels. Xu et al. found that phloretin, a plant-derived natural product, is an important molecule for the treatment of gastric cancer. To investigate its apoptotic effect, 4, 8, $16 \mu \mathrm{M}$ of phloretin was treated with AGS cells for $24 \mathrm{~h}$. The apoptotic AGS cells increased from $1.25 \%$ in control to $46.3 \%$ at $40 \mu \mathrm{M}$ doses of phloretin. Expression of Bax was increased in a dose-dependently while the expression of Bcl-2 decreased [55]. Podophyllotoxin, extracted from Linum album Kotschy (Linaceae), induced apoptosis and downregulated zinc finger protein 703 oncogene expression according to a study by Akbari et al. [56]. When AGS cells were treated with different concentrations $(200,400,600,800$ and $1000 \mu \mathrm{g} / \mathrm{mL}$ ) for $24 \mathrm{~h}$, apoptotic cell count was increased $44 \%$ in contrast to $2.70 \%$ in untreated AGS cells. Tsai et al. found that 7-acetylsinumaximol B (7-AB), discovered from Sinularia sandensis (Alcyoniidae), shows anti-proliferative effect through apoptosis against human gastric carcinoma NCI-N87 cells [57]. In the study, the cells were treated with 4,8 , $16 \mu \mathrm{M}$ 7-AB formula for $24 \mathrm{~h}$. 7-AB treatment increased the expression of Bad, Bcl-like protein 11 (Bim), Bax, and cytochrome c, decreased the expression levels of phosphorylated Bad (p-Bad), myeloid cell lukemia-1 (Mcl-1), Bcl-xL, and Bcl-2 proteins. Apoptosis was induced by elevated levels of ROS and caspase-3 along with inhibition of Bcl-2, and loss of $\Delta \Psi \mathrm{m}$. Crosolic acid, isolated from Actinidia valvata Dunn. Radix (Actinidiaceae), was reported to inhibit proliferation of BGC-823 cells by downregulating the NF- $\kappa$ B pathway

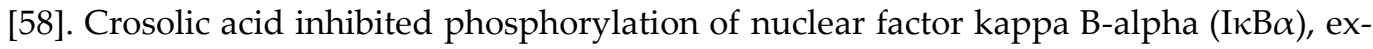
pression of p65, and nuclear translocation and DNA-binding activity of NF- $\kappa \mathrm{B}$. Upregulation of Bax and downregulation of Bcl-2 were also observed. Jung et al. found that curcuzedoalide, sesquiterpene components of Curcuma zedoaria Roscoe Rhizoma (Zingiberaceae) induced mitochondrial apoptosis of AGS cells [59]. AGS cells were treated with curcuzedoalide at the concentration of $100 \mu \mathrm{M}$ and $200 \mu \mathrm{M}$ for $24 \mathrm{~h}$. Exposure to curcuzedoalide induced cleavage of PARP as well as caspase-8, caspase- 9 , and caspase- 3 , in a dosedependent manner. Deacetylisovaltratum, extracted from Patrinia heterophylla Bunge. (Caprifoliaceae, was treated to AGS and HGC-27 human gastric cancer cell lines for $24 \mathrm{~h}$ at 
the dose of $4,8,16 \mu \mathrm{M} / \mathrm{mL}$ and $10,20,30 \mu \mathrm{M} / \mathrm{mL}$, respectively [60]. Deacetylisovaltratum induced mitochondrial and caspase-dependent apoptosis. Li et al. demonstrated that elemene counters against gastric cancer via regulation of the ERK $1 / 2$ signaling pathway [61]. Elemene is a sesquiterpenoid mixture extracted from a traditional herbal medicine Curcuma zedoaria Roscoe Rhizoma (Zingiberaceae). In an in vitro investigation, BGC-823 cells were treated with 20,40,80,160 $\mathrm{g} / \mathrm{mL}$ elemene solution for $24 \mathrm{~h}$. Moreover, Bak, Bax, and Cyclophilin D (CypD) expressions increased while Bcl-2, Bcl-xL and XIAP levels decreased. Grifolin, isolated from the mushroom Albatrellus confluens (Alb. \& Schwein.) Kotl. \& Pouzar (Albatrellaceae), inhibited growth and invasion of gastric cancer cells by inducing apoptosis and suppressing the ERK1/2 pathway [62]. Liao et al. reported that N-butylidenephthalide (BP), a bioactive compound of Angelica Sinensis Diels Radix, activated the intrinsic apoptotic pathway of human gastric cancer cells AGS, NCI-N87 and TSGH-9201 [63]. Lyu et al. reported anticancer activities of paeonol, a principal active component found root bark of Paeonia suffruticosa Andr. Cortex (Paeoniaceae) and Cynachum paniculatum K. Schum Radix (Apocynaceae) [64]. Paeonol treatment (0.1, 0.2, $0.4 \mathrm{mg} / \mathrm{mL}$ for $48 \mathrm{~h}$ ) inhibited proliferation, invasion, migration and induced apoptosis against BGC823 cells. The protein expression of matrix metalloproteinase (MMP)-2 and MMP-9 were attenuated in a concentration-dependent manner by paeonol. Pseudolaric acid B inhibited cell proliferation and induced apoptosis of SGC7901/ADR cells, a multidrug-resistant gastric cancer cell line [65]. Pseudolaric acid B is a diterpene acid isolated from Pseudolarix, the dried root bark of Pseudolarix kaempferi Gorden Cortex (Pinaceae). The study revealed that p53 and Bax were activated while the expressions of P-glycoprotein (P-gp), cyclooxygenase 2 (COX-2), Bcl-2 and Bcl-xL were inhibited. Downregulation of phosphorylated Akt, Bcl-2 and upregulation of caspase-3 and Bax were detected. Thymol is a phenolic compound isolated from Thymus quinquecostatus Celak. (Lamiaceae) that possesses anti-inflammatory, anticancer, antibacterial, and more biological efficacies [66]. Thymol showed cytotoxicity on AGS cancer cells via intrinsic mitochondrial pathway when treated at doses of 100, 200, $400 \mu \mathrm{M}$ for 6, 12 and $24 \mathrm{~h}$. It upregulated Bax, PARP expressions and promoted cleavage of caspase-7, caspase- 8 and caspase-9 and downregulated $\Delta \Psi \mathrm{m}$. The anticancer potencies of toosendanin (TSN), a triterpenoid extracted from Melia toosendan Sieb et Zucc Cortex et Fructus (Meliaceae), was discussed in two studies. According to Wang et al., SGC-7901 treated with 0.5 or $1 \mu \mathrm{M}$ TSN solution for 24, $48 \mathrm{~h}$ demonstrated a dose-dependent increase of cells undergoing early apoptosis [67]. Following facilitation of microRNA 200a, or miR-200a, a microRNA that targets $\beta$-catenin, TSN inactivated the $\beta$-catenin pathway in SGC-7901 cells and subsequently induced apoptosis. Moreover, the compound inhibited migration, invasion and TGF- $\beta 1$-induced epithelial-mesenchymal transition (EMT). Similarly, Zhou et al. reported that TSN inhibited cell proliferation and induced caspase-dependent apoptosis via the p38 MAPK signaling pathway [68]. Incarvillea compacta has been used to treat stomach disease in Tibet for many years. Incarvillea compacta Radix extract $(5,10,20 \mu \mathrm{g} / \mathrm{mL})$ was treated on EBV-positive AGS cells for $24 \mathrm{~h}$. Apoptosis was induced by disruption of mitochondrial membrane potential, activation of Bax, and inactivation of Bcl-2. Arsenic sulfide, a compound of natural mineral realgar [69], induced apoptosis of human gastric cancer AGS and MGC-803 cells. Expression levels of p53 and murine double minute 2 (MDM2) expression were elevated, and proteins mediating the intrinsic pathway of apoptosis were regulated. Kapoor and Dharmesh reported that peptic oligosaccharide separated from Solanum lycopersicum L. (Solanaceae) induced apoptosis by suppressing galectin-3 expressions [70].

Additionally, several natural products retarded tumor growth in animal models (Table 2). Wu et al. revealed that phenolic alkaloids of Menispermum dauricum induced apoptosis and suppressed gastric tumor growth by inducing apoptosis and inhibiting oncogenic Kirsten Rat sarcoma viral oncogene homolog (K-RAS) expression [42]. Xenograft mice treated with trifolirhizin, DDMN or arsenic trioxide developed smaller tumors compared to the control group [44, 48, 69]. When BALB/C mice grafted with MFC mouse gas- 
tric cancer cells were treated curcumin solution every day for 60 days, expressions of interferon gamma (IFN- $\gamma)$, tumor necrosis factor-alpha (TNF- $\alpha)$, granzyme B, and perforin were upregulated while differentiated embryonic chondrocyte gene 1 (DEC1), hypoxiainducible factor-1 alpha (HIF-1 $\alpha$ ), STAT3 and VEGF expressions were downregulated in the experimental group [71]. When MKN45 treated BALB/ca mice were treated with LicA, tumor growth was significantly inhibited in contrast to the vehicle group without LicA treatment [53]. Elemene retarded tumor growth in nude mice and showed better efficacy when synergized with PD98059 [61]. In a xenograft mouse model, mice treated with grifolin survived for a longer period compared to the control group [62]. 
Table 1. Apoptosis-inducing natural compounds in vitro.

\begin{tabular}{|c|c|c|c|c|c|c|c|}
\hline Classification & Compound/ Extract & Source & $\begin{array}{c}\text { Experimental } \\
\text { Model }\end{array}$ & Dose; Duration & Efficacy & Mechanism & References \\
\hline Alkaloids & Berberine & $\begin{array}{c}\text { (family: Ranunculaceae) } \\
\text { Coptidis japonica Makino Rhi- } \\
\text { zoma }\end{array}$ & SGC-7901 & $\begin{array}{c}5,10,20 \mu \mathrm{M} ; 24, \\
48 \mathrm{~h}\end{array}$ & Induction of apoptosis & & {$[38]$} \\
\hline Alkaloids & Cyclovirobuxine D & $\begin{array}{c}\text { (family: Buxaceae) } \\
\text { Buxus microphylla Richardii } \\
\text { Radix }\end{array}$ & $\begin{array}{c}\text { MGC-803, MKN- } \\
28\end{array}$ & $\begin{array}{l}30,60,120 \\
\mu \mathrm{M} / \mathrm{L} ; 48 \mathrm{~h}\end{array}$ & Induction of apoptosis & $\begin{array}{c}\uparrow c \text {-caspase-3, Bax } \\
\downarrow \text { Bcl-2 }\end{array}$ & [39] \\
\hline Alkaloids & GFG-3a & $\begin{array}{c}\text { (family: Meripilaceae) } \\
\text { Grifola frondose (Diks.) Gray } \\
\text { Mycelia }\end{array}$ & SGC-7901 & $\begin{array}{c}100,200 \mu \mathrm{g} / \mathrm{mL} \\
24,48 \mathrm{~h}\end{array}$ & Induction of apoptosis & $\begin{array}{c}\uparrow \mathrm{RBBP} 4, \text { caspase-3, } \\
-8, \mathrm{p} 53, \mathrm{Bax}, \mathrm{Bad} \\
\downarrow \text { RUVBL, NPM, } \\
\text { Bcl-2, Bcl-xL, PI3K, } \\
\text { Akt1 }\end{array}$ & {$[40]$} \\
\hline Alkaloids & Melittin & $\begin{array}{c}\text { (family: Apidae) } \\
\text { Apis cerena } \text { Fabricius venom }\end{array}$ & SGC-7901 & $\underset{\mathrm{h}}{4 \mu \mathrm{g} / \mathrm{mL} ; 1,2,4}$ & Induction of apoptosis & $\begin{array}{c}\uparrow \text { caspase-3, cyt c, } \\
\text { Endo G, AIF, } \\
\text { Smac/Diablo, ROS } \\
\downarrow \Delta \Psi \mathrm{m}\end{array}$ & [41] \\
\hline Alkaloids & Phenolic alkaloids & $\begin{array}{c}\text { (family: Menispermaceae) } \\
\text { Menispermum dauricum DC. } \\
\text { Rhizoma }\end{array}$ & SGC-7901 & $\begin{array}{c}5,10,20 \mu \mathrm{M} ; \\
24 \mathrm{~h}\end{array}$ & Induction of apoptosis & $\begin{array}{c}\uparrow \text { Bax, caspase-3 } \\
\downarrow \text { K-RAS }\end{array}$ & {$[42]$} \\
\hline $\begin{array}{l}\text { Alkaloids, } \\
\text { Terpenoids }\end{array}$ & $\begin{array}{l}\text { Berberine, } \\
\text { d-Limonene }\end{array}$ & $\begin{array}{c}\text { (1) } \\
\text { (family: Ranunculaceae) } \\
\text { Coptidis japonica Makino Rhi- } \\
\text { zoma } \\
\text { (2) (family: Rutaceae) } \\
\text { Evodiae rutaecarpa } \text { Bentham. }\end{array}$ & MGC-803 & $\begin{array}{l}\text { (1) } 20 \mu \mathrm{M} ; 24 \text {, } \\
36,48 \mathrm{~h} \\
\text { (2) } 80 \mu \mathrm{M} ; 24, \\
36,48 \mathrm{~h}\end{array}$ & Induction of apoptosis & $\begin{array}{c}\uparrow \mathrm{ROS}, \text { caspase-3 } \\
\uparrow \Delta \Psi \mathrm{m}, \mathrm{Bcl}-2\end{array}$ & [43] \\
\hline
\end{tabular}




\begin{tabular}{|c|c|c|c|c|c|c|c|}
\hline Flavonoids & Trifolirhizin & $\begin{array}{l}\text { (family: Fabaceae) Sophora } \\
\text { flavescens Aiton Radix }\end{array}$ & MKN-45 & $\begin{array}{c}20,30,40 \\
\mu \mathrm{g} / \mathrm{mL} ; 48 \mathrm{~h}\end{array}$ & Induction of apoptosis & $\begin{array}{l}\uparrow \text { caspase-9, -3, c- } \\
\text { PARP, p53, p38 } \\
\downarrow \text { EGFR, CDC2, cy- } \\
\text { clin B, } \Delta \Psi \mathrm{m}\end{array}$ & [44] \\
\hline Phytosterols & Ginsenoside - Rh2 & $\begin{array}{l}\text { (family: Araliaceae) Panax } \\
\text { ginseng C.A. Mey Radix }\end{array}$ & SGC-7901 & $\begin{array}{c}5,10,20 \mu \mathrm{g} / \mathrm{mL} \\
24,48 \mathrm{~h}\end{array}$ & Induction of apoptosis & $\uparrow \mathrm{Bax} \downarrow \mathrm{Bcl}-2$ & [45] \\
\hline Phytosterols & Periplocin & $\begin{array}{l}\text { (family: Apocynaceae) Perip- } \\
\text { locae sepium Bunge. }\end{array}$ & $\begin{array}{l}\text { SGC-7901, MGC- } \\
803, \text { BGC-823 }\end{array}$ & $\begin{array}{c}50,100,200 \\
\mathrm{ng} / \mathrm{mL} ; 24,48 \mathrm{~h}\end{array}$ & Induction of apoptosis & $\begin{array}{c}\uparrow \text { Mcl-1, c-caspase-3, } \\
\text { EGR } 1 \\
\downarrow \text { pro-Bid, p-ERK 1/2 }\end{array}$ & {$[72]$} \\
\hline Phytosterols & Tanshinone IIA & $\begin{array}{l}\text { (family: Lamiaceae) Salviae } \\
\text { miltiorrhiza Bunge. Radix }\end{array}$ & AGS & $\begin{array}{c}2.0,3.7,5.5 \\
\mu \mathrm{g} / \mathrm{mL} ; 24,48 \mathrm{~h}\end{array}$ & Induction of apoptosis & $\begin{array}{c}\uparrow T N F-\alpha, \text { Fas, p-p38, } \\
\text { p-JNK, p53, p21, } \\
\text { caspase-8, -3 } \\
\downarrow \text { p-ERK, CDC2, cy- } \\
\text { clin A, cyclin B1 }\end{array}$ & [46] \\
\hline Polyphenols & [6]-Gingerol & $\begin{array}{l}\text { (family: Zingiberaceae) Zingi- } \\
\text { ber officinale Roscoe Rhizoma }\end{array}$ & AGS & $\begin{array}{c}100,250 \mu \mathrm{M} ; 24 \\
\mathrm{~h}\end{array}$ & Induction of apoptosis & $\begin{array}{c}\uparrow \text { cyt c, Bax } \\
\downarrow \text { Bcl-2 }\end{array}$ & [47] \\
\hline Polyphenols & $\begin{array}{l}\text { 2,7-dihydroxy-3- } \\
\text { methylanthraquinone } \\
\text { (DDMN) }\end{array}$ & $\begin{array}{l}\text { (family: Rubiaceae) Hedyotis } \\
\text { diffusa Wild Herba }\end{array}$ & SGC-7901 & $\begin{array}{c}10,20,40 \mu \mathrm{M} \\
48 \mathrm{~h}\end{array}$ & $\begin{array}{l}\text { Inhibition of prolifera- } \\
\text { tion }\end{array}$ & $\begin{array}{c}\uparrow \text { Bax, Bad, caspase- } \\
3,-9 \text {, cyt c } \\
\downarrow \text { Bcl-xL, Bcl-2 }\end{array}$ & [48] \\
\hline Polyphenols & $\begin{array}{c}\text { 6, 7, 30-trimethoxy-3, } \\
5,40 \\
\text {-trihydroxy flavone } \\
\text { (TTF) }\end{array}$ & $\begin{array}{c}\text { (family: Saxifragaceae) } \\
\text { Chrysosplenium nudicaule } \\
\text { Ledeb Herba }\end{array}$ & SGC-7901 & $\begin{array}{c}2,4,8,16,32 \\
\mu \mathrm{g} / \mathrm{mL} ; 24,48 \\
72 \mathrm{~h}\end{array}$ & Induction of apoptosis & $\begin{array}{c}\uparrow \text { endogenous } \mathrm{Ca} 2+\text { / } \\
\mathrm{Mg} 2+\text { dependent en- } \\
\text { donuclease }\end{array}$ & [49] \\
\hline Polyphenols & Curcumin & $\begin{array}{c}\text { (family: Zingiberaceae) } \\
\text { Curcuma longa L. Rhizoma }\end{array}$ & $\begin{array}{l}\text { SGC-7901, BGC- } \\
823\end{array}$ & $\begin{array}{c}5,10,15,20,40 \\
\mu \mathrm{M} / \mathrm{L} ; 24 \mathrm{~h}\end{array}$ & Induction of apoptosis & $\begin{array}{c}\downarrow \mathrm{XIAP} \\
\uparrow \mathrm{miR}-33 \mathrm{~b}\end{array}$ & [50] \\
\hline
\end{tabular}




\begin{tabular}{|c|c|c|c|c|c|c|c|}
\hline Polyphenols & Esculetin & $\begin{array}{l}\text { (family: Asteraceae) Artemesia } \\
\text { scoparia Waldst. et Kit, Ar- } \\
\text { temesia capillaris Thunb., } \\
\text { (family: Plumbaginaceae) } \\
\text { Ceratostigma willmottianum } \\
\text { Stapf, (family: Rutaceae) Cit- } \\
\text { rus limon Osbeck Folium }\end{array}$ & $\begin{array}{l}\text { SGC-7901, MGC- } \\
\text { 803, BGC-823 }\end{array}$ & $\begin{array}{c}12.5,25,50 \mu \mathrm{M} \\
24 \mathrm{~h}\end{array}$ & Induction of apoptosis & $\begin{array}{c}\uparrow \text { ROS, c-caspase-9, - } \\
\text { 3, c-PARP, cyt c, } \\
\text { Bak, Bax, CypD } \\
\downarrow \text { Bcl-2, Bcl-xL, } \\
\text { XIAP }\end{array}$ & {$[73]$} \\
\hline Polyphenols & $\begin{array}{l}\text { Hydroxysafflor Yellow } \\
\text { A }\end{array}$ & $\begin{array}{l}\text { (family: Asteraceae) Cartha- } \\
\text { mus tinctorius L. }\end{array}$ & BGC-823 & $100 \mu \mathrm{M} ; 48 \mathrm{~h}$ & Induction of apoptosis & $\uparrow$ caspase- 3, PPAR $\gamma$ & {$[51]$} \\
\hline Polyphenols & $\begin{array}{c}\text { Kurarinone } \\
\text { (combined with } \\
\text { TRAIL) }\end{array}$ & $\begin{array}{l}\text { (family: Fabaceae) Sophora } \\
\text { flavescens Aiton Radix }\end{array}$ & SGC-7901 & $5 \mu \mathrm{M} ; 24 \mathrm{~h}$ & $\begin{array}{c}\text { Enhancement of } \\
\text { TRAIL-induced apopto- } \\
\text { sis }\end{array}$ & $\begin{array}{l}\downarrow \text { Mcl-1, c-FLIP, p- } \\
\text { STAT3 }\end{array}$ & {$[52]$} \\
\hline Polyphenols & Licochalcone A & $\begin{array}{l}\text { (family: Fabaceae) Glycyr- } \\
\text { rhiza glabra L. Root }\end{array}$ & $\begin{array}{c}\text { MKN-45, SGC- } \\
7901\end{array}$ & $\begin{array}{l}15,30,60 \mu \mathrm{M} \\
24 \mathrm{~h}\end{array}$ & $\begin{array}{l}\text { Inhibition of cell prolif- } \\
\text { eration and tumor gly- } \\
\text { colysis }\end{array}$ & $\begin{array}{c}\uparrow c-\text { caspase-3, c- } \\
\text { PARP } \\
\downarrow \text { Bcl-2, Mcl-1, HK2, } \\
\text { p-Akt, p-ERK1/2, p- } \\
\text { S6, p-GSK3 } \beta\end{array}$ & {$[53]$} \\
\hline Polyphenols & Ophiopogonin B & $\begin{array}{c}\text { (family: Asparagaceae) } \\
\text { Ophiopogon japonicus Thunb } \\
\text { Root }\end{array}$ & SGC-7901 & $5,10,20 \mu \mathrm{M}$ & Induction of apoptosis & $\begin{array}{c}\uparrow \mathrm{ROS}, \mathrm{Bax}, \text { caspase- } \\
3 \\
\downarrow \text { p-ERK } 1 / 2, \mathrm{p}-\mathrm{JNK} \\
1 / 2, \Delta \Psi \mathrm{m}, \mathrm{Bcl}-2\end{array}$ & {$[54]$} \\
\hline Polyphenols & Phloretin & & AGS & 4. $8,16 \mu \mathrm{M} ; 24 \mathrm{~h}$ & $\begin{array}{l}\text { Induction of apoptosis } \\
\text { Inhibition of invasion }\end{array}$ & $\uparrow \mathrm{Bax} \downarrow$ Bcl-2 & {$[55]$} \\
\hline Polyphenols & Podophyllotoxin & $\begin{array}{c}\text { (family: Linaceae) } \\
\text { Linum album Kotschy }\end{array}$ & AGS & $\begin{array}{l}200,400,600 \\
800,1000 \\
\mu \mathrm{g} / \mathrm{mL} ; 24 \mathrm{~h}\end{array}$ & Induction of apoptosis & $\downarrow$ ZNF703 & {$[56]$} \\
\hline Terpenoids & 7-Acetylsinumaximol B & $\begin{array}{c}\text { (family: Alcyoniidae) Sinularia } \\
\text { sandensis }\end{array}$ & NCI-N87 & $4,8,16 \mu \mathrm{M} ; 24 \mathrm{~h}$ & Induction of apoptosis & $\begin{array}{c}\uparrow \mathrm{Bad}, \mathrm{Bim}, \mathrm{Bax}, \mathrm{cyt} \\
\mathrm{c} \\
\downarrow \mathrm{p}-\mathrm{Bad}, \mathrm{Mcl}-1, \mathrm{Bcl}-\end{array}$ & {$[57]$} \\
\hline
\end{tabular}




\begin{tabular}{|c|c|c|c|c|c|c|c|}
\hline Terpenoids & Crosolic Acid & $\begin{array}{c}\text { (family: Actinidiaceae) } \\
\text { Actinidia valvata Dunn Radix }\end{array}$ & BGC-823 & $\begin{array}{c}20,40,80 \\
\mu \mathrm{g} / \mathrm{mL} ; 72 \mathrm{~h}\end{array}$ & Induction of apoptosis & 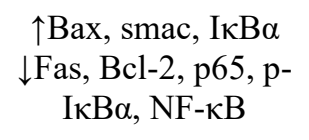 & {$[58]$} \\
\hline Terpenoids & $\begin{array}{c}\text { Curcuzedoalide / Metha } \\
\text { tract }\end{array}$ & $\begin{array}{l}\text { (family: Zingibera- } \\
\text { ceae) } \\
\text { Curcuma zedoaria } \\
\text { Roscoe Rhizoma }\end{array}$ & AGS & $\begin{array}{c}100,200 \mu \mathrm{M} ; 24 \\
\mathrm{~h}\end{array}$ & Induction of apoptosis & $\begin{array}{c}\uparrow c-\text { caspase- } 8,-9,-3, \\
\text { c-PARP }\end{array}$ & {$[59]$} \\
\hline Terpenoids & Deacetylisovaltratum & $\begin{array}{c}\text { (family: Caprifoliaceae) } \\
\text { Patrinia heterophylla Bunge. }\end{array}$ & $\begin{array}{c}\text { (1) AGS } \\
\text { (2) HGC-27 }\end{array}$ & $\begin{array}{c}\text { (1) } 4,8,16 \mu \mathrm{M} \text {; } \\
24 \mathrm{~h} \\
\text { (2) } 10,20,30 \\
\mu \mathrm{M} ; 24 \mathrm{~h}\end{array}$ & Induction of apoptosis & $\begin{array}{c}\uparrow \mathrm{p} 21, \text { caspase-3, c- } \\
\text { PARP } \\
\downarrow \text { p-STAT3, pro- } \\
\text { caspase- } 9, \Delta \Psi \mathrm{m}\end{array}$ & {$[60]$} \\
\hline Terpenoids & Elemene & $\begin{array}{c}\text { (family: Zingiberaceae) } \\
\text { Curcuma zedoaria } \text { Roscoe } \\
\text { Rhizoma }\end{array}$ & BGC-823 & $\begin{array}{l}20,40,80,160 \\
\mu \mathrm{g} / \mathrm{mL}: 24 \mathrm{~h}\end{array}$ & Induction of apoptosis & $\begin{array}{c}\uparrow \text { Bax, p-ERK } 1 / 2 \\
\downarrow \text { Bcl-2 }\end{array}$ & {$[61]$} \\
\hline Terpenoids & Grifolin & $\begin{array}{c}\text { (family: Albatrellaceae) } \\
\text { Albatrellus confluens (Alb. \& } \\
\text { Schwein.) Kotl. \& Pouzar }\end{array}$ & $\begin{array}{l}\text { BGC-823, SGC- } \\
7901\end{array}$ & $10,50 \mu \mathrm{M} ; 48 \mathrm{~h}$ & Induction of apoptosis & $\begin{array}{c}\uparrow \text { caspase-9, }-3, \\
\text { CDKN2 } \\
\downarrow \text { MEK1, MEKK3 } \\
\text { MEK5 }\end{array}$ & [62] \\
\hline Terpenoids & N-butylidenephthalide & $\begin{array}{l}\text { (family: Apiaceae) Angelica } \\
\quad \text { Sinensis Diels Radix }\end{array}$ & AGS & $\begin{array}{l}25,50,75 \\
\mu \mathrm{g} / \mathrm{mL} ; 24 \mathrm{~h}\end{array}$ & Induction of apoptosis & $\begin{array}{l}\uparrow \text { REDD1 } 1 \\
\downarrow \mathrm{mTOR}\end{array}$ & {$[63]$} \\
\hline Terpenoids & Paeonol / extract & $\begin{array}{l}\text { (family: Paeoniaceae) Paeonia } \\
\text { suffruticosa Andr Root bark, } \\
\text { (family: Apocynaceae) Cynan- } \\
\text { chum paniculatum K. Schum } \\
\text { Radix }\end{array}$ & BGC-823 & $\begin{array}{c}0.1,0.2,0.4 \\
\mathrm{mg} / \mathrm{mL} ; 24,48 \mathrm{~h}\end{array}$ & $\begin{array}{l}\text { Inhibition of prolifera- } \\
\text { tion, invasion, and mi- } \\
\text { gration } \\
\text { Induction of apoptosis }\end{array}$ & $\downarrow$ MMP-2, -9 & [64] \\
\hline
\end{tabular}


(family: Pinaceae) Pseu-

Terpenoids Pseudolaric acid B dolarix kaempferi Gorden Root bark
$5,10,20 \mu \mathrm{M} / \mathrm{L}$

$24 \mathrm{~h}$

Induction of apoptosis

$\uparrow p 53$, Bax

P-gp, COX-2, Bcl-

$2, \mathrm{Bcl}-\mathrm{xL}$

$\uparrow$ Bax, c-PARP,

$100,200,400$
$\mu \mathrm{M} ; 6,12,24 \mathrm{~h} \quad$ Induction of apoptosis $\begin{gathered}\text { caspase- } 8, \text { caspase-7, } \\ \text { caspase-9 }\end{gathered}$

caspase-9

$\downarrow \Delta \Psi \mathrm{m}$
Terpenoids

Thymol

Thymus quinquecostatus Celak Essential oil
AGS

$\mu \mathrm{M} ; 6,12,24 \mathrm{~h}$

(family: Meliaceae)

Terpenoids Toosendanin

Melia toosendan Sieb et zucc

SGC-7901

$0.5,1 \mu \mathrm{M} ; 48 \mathrm{~h}$

Inhibition of invasion,

migration and EMT

Induction of apoptosis

$\uparrow$ E-cadherin

$\frac{\downarrow \beta \text {-catenin }}{\uparrow \mathrm{miR}-200 \mathrm{a}}$

$\uparrow \mathrm{miR}-200 \mathrm{a}$

[65]

$\uparrow$ c-caspase- $3,-8,-9$,

c-PARP, Bax, p-p38

$\downarrow$ Bcl-2, Bcl-xL, Mcl-

1 , survivin, XIAP
Terpenoids Toosendanin Melia toosendan Sieb et Zucc

Cortex or Fructus
(1) AGS

(2) $\mathrm{HGC}-27$
(1) $0.5,1,2 \mu \mathrm{M}$; $48 \mathrm{~h}$

(2) $0.5,1,2 \mu \mathrm{M}$; $36 \mathrm{~h}$
Inhibition of proliferation

Induction of apoptosis
$0.31,0.62,1.25$,
$2.5,5,10 \mu \mathrm{M}$;

$24,48,72 \mathrm{~h}$
Induction of apoptosis 个p53, Bax, MDM2

$\downarrow \Delta \Psi \mathrm{m}, \mathrm{Bcl}-2$
Arsenic sulfide

Realgar
AGS, MGC-803 (family: Solanaceae)

Solanum lycopersicum L.
AGS
$10,20,30$

$\mu \mathrm{g} / \mathrm{mL} ; 48 \mathrm{~h}$
Induction of apoptosis

$\downarrow$ Galectin-3

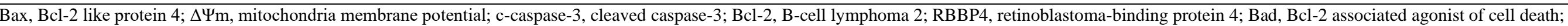

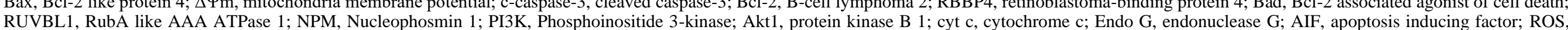

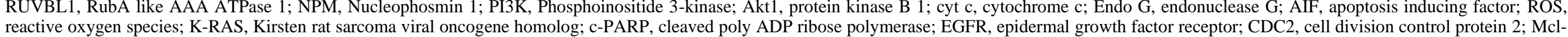

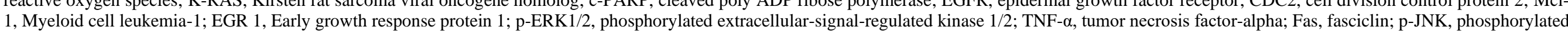

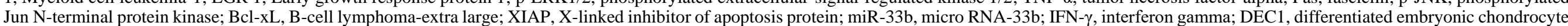

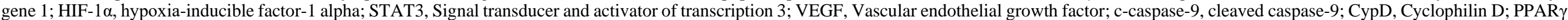

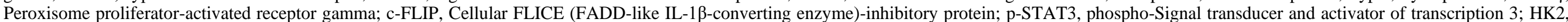

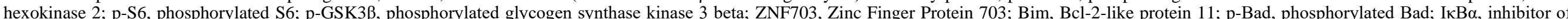

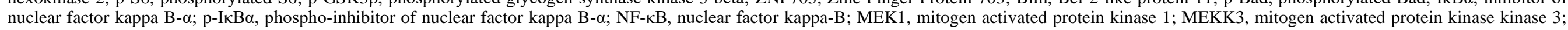




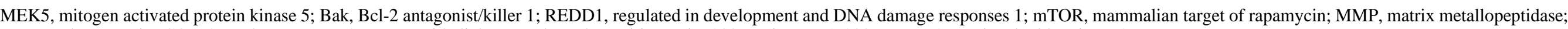
P-gp, P-glycoprotein; COX-2, cyclooxygenase 2; EMT, epithelial-mesenchymal transition; miR-200a, MicroRNA 200a; MDM2, murine double minute 2

Table 2. Apoptosis-inducing natural compounds in vivo.

\begin{tabular}{|c|c|c|c|c|c|c|c|}
\hline Classification & Compound/ Extract & Source & $\begin{array}{l}\text { Experimental } \\
\text { Model }\end{array}$ & $\begin{array}{l}\text { Dose; Dura- } \\
\text { tion }\end{array}$ & Efficacy & Mechanism & References \\
\hline Alkaloids & Phenolic alkaloids & $\begin{array}{c}\text { (family: Menispermaceae) } \\
\text { Menispermum dauricum DC. } \\
\text { Rhizoma }\end{array}$ & $\begin{array}{l}\text { Nude mice / SGC- } \\
7901\end{array}$ & $\begin{array}{l}5,10,20 \\
\mathrm{mg} / \mathrm{kg} / \text { week; } 3 \\
\text { weeks }\end{array}$ & $\begin{array}{l}\text { Suppression of tumor } \\
\text { growth }\end{array}$ & & {$[42]$} \\
\hline Flavonoids & Trifolirhizin & $\begin{array}{c}\text { (family: Fabaceae) } \\
\text { Sophora flavescens Aiton. Ra- } \\
\text { dix }\end{array}$ & $\begin{array}{l}\text { BALB/C nude mice } \\
\quad / \mathrm{MKN}-45\end{array}$ & $\begin{array}{l}1-3 \mathrm{mg} / \mathrm{kg} ; 3 \\
\text { weeks }\end{array}$ & $\begin{array}{l}\text { Retardation of tumor } \\
\text { growth }\end{array}$ & $\uparrow \mathrm{c}$-caspase-3 $\downarrow \Delta \Psi \mathrm{m}$ & {$[44]$} \\
\hline Polyphenols & $\begin{array}{l}\text { 2,7-dihydroxy-3- } \\
\text { methylanthraquinone } \\
\text { (DDMN) }\end{array}$ & $\begin{array}{c}\text { (family: Rubiaceae) } \\
\text { Hedyotis diffusa Wild. Herba }\end{array}$ & $\begin{array}{l}\text { nude mice / SGC- } \\
7901\end{array}$ & $\begin{array}{l}40 \mathrm{mg} / \mathrm{kg} ; 5 \\
10,15,20 \text { days }\end{array}$ & $\begin{array}{l}\text { Inhibition of gastric } \\
\text { cancer cell growth }\end{array}$ & $\begin{array}{c}\uparrow \text { Bax, Bad, c-caspase- } \\
3,-9, \text { cyt c } \\
\downarrow \text { Bcl-xL, Bcl-2 }\end{array}$ & {$[48]$} \\
\hline Polyphenols & Curcumin & $\begin{array}{l}\text { (family: Zingiberaceae) } \\
\text { Curcuma longa L. Rhizoma }\end{array}$ & $\begin{array}{l}\mathrm{BALB} / \mathrm{C} \text { mice / } \\
\mathrm{MFC}\end{array}$ & $\begin{array}{c}20,40,60 \\
\mu \mathrm{M} / \mathrm{L} / \text { day; } 60 \\
\text { days }\end{array}$ & $\begin{array}{l}\text { Inhibition of tumor } \\
\text { growth } \\
\text { Induction of apoptosis } \\
\text { Activation of immune } \\
\text { cells }\end{array}$ & $\begin{array}{l}\uparrow \text { IFN- } \gamma, \text { TNF- } \alpha, \\
\text { granzyme B, perforin } \\
\downarrow \text { DEC } 1, \text { HIF- } 1 \alpha, \\
\text { STAT3, VEGF }\end{array}$ & {$[71]$} \\
\hline Polyphenols & Licochalone A & $\begin{array}{c}\text { (family: Fabaceae) } \\
\text { Glycyrrhiza glabra L. Radix }\end{array}$ & $\begin{array}{l}\text { BALB/ca nude } \\
\text { mice / MKN-45 }\end{array}$ & $\begin{array}{l}10 \mathrm{mg} / \mathrm{kg} / \text { day; } \\
33 \text { days }\end{array}$ & $\begin{array}{l}\text { Inhibition of tumor } \\
\text { growth }\end{array}$ & & {$[53]$} \\
\hline Terpenoids & Elemene & $\begin{array}{l}\text { (family: Zingiberaceae) } \\
\text { Curcuma longa L. Rhizoma }\end{array}$ & $\begin{array}{l}\text { BALB/c athymic } \\
\text { nude mice / BGC- } \\
823\end{array}$ & $\begin{array}{l}200 \\
\mathrm{mg} / \mathrm{kg} / \text { day; } 15 \\
\text { days }\end{array}$ & $\begin{array}{l}\text { Retardation of tumor } \\
\text { growth }\end{array}$ & & {$[61]$} \\
\hline Terpenoids & Grifolin & $\begin{array}{c}\text { (family: Albatrellaceae) } \\
\text { Albatrellus confluens (Alb. \& }\end{array}$ & $\begin{array}{l}\text { Balb/c nude mice / } \\
\text { BGC-823, SGC- }\end{array}$ & $\begin{array}{c}15 \mathrm{mg} / \mathrm{kg} ; 2 \\
\text { days }\end{array}$ & $\begin{array}{l}\text { Improvement of sur- } \\
\text { vival time }\end{array}$ & & {$[62]$} \\
\hline
\end{tabular}


Schwein.) Kotl. \& Pouzar

7901
Realgar
$\mathrm{BALB} / \mathrm{c}-\mathrm{nu} / \mathrm{nu}$
mice / MGC-803
$1,2 \mathrm{mg} / \mathrm{kg} ; 3$
weeks

Induction of apoptosis

$\uparrow \mathrm{p} 53$

[69]

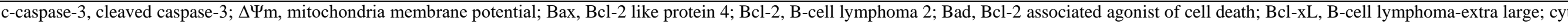

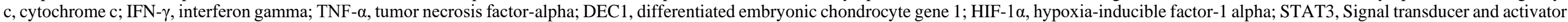
of transcription 3; VEGF, Vascular endothelial growth factor. 


\subsubsection{Natural plant extracts inducing apoptosis in gastric cancer}

Several have been studies demonstrated that natural product extracts of plant or animal sources induced apoptosis against gastric cancer cells and inhibited tumor growth of xenograft animals (Table 3, 4). Acetonic extracts of the endolichenic fungus EL002332 isolated from Ribes nigrum L. (Saxifragaceae) showed selective cytotoxicity against AGS human gastric cancer cells [74]. Liu et al. reported that the polyphenol-rich extract of Ribes nigrum L. (Saxifragaceae), or black currant, induced apoptosis of MKN-45 cells via MAPKand PI3K/Akt-mediated mitochondrial pathways [75]. Euphorbia lunulata Bunge. (Euphorbiaceae) ethanol and n-hexane extract was processed to 10,20 , and $40 \mu \mathrm{g} / \mathrm{mL}$ solutions and applied to SGC-7901 and ADR cells for $24 \mathrm{~h}$ [76]. The solution increased the expression of Bax, caspase-3, caspase-8, and caspase-9, and suppressed the expression of Bcl-2, thereby inducing apoptosis. Xie et al. reported that treatment of ethanolic extract of Cordyceps cicadae (Miq.) Massee. (EEC), a member of the Cordyceps genus, induced death-receptor mediated and mitochondria-mediated apoptosis [77]. Hao et al. reported that ethanol extract of Smilax glabra Roxb. (Smilacaceae) induced apoptosis by regulating Akt-mediated signaling pathways [78]. The root extract of Chinese tonic herb Astragulus cornus mass L. radix (Cornaceae) contains astragalosides, flavonoids and polysaccharides [79]. Wang et al. demonstrated that astragalus induced apoptosis by inhibiting the IL-6/STAT3 signaling pathway. SGC-7901 gastric cancer cells treated with astragalus extract (AE) showed downregulation of interleukin-6 (IL-6) and STAT3. To investigate the efficacy of AE in vivo, AE (60 or $120 \mathrm{mg} / \mathrm{kg}$, daily) was treated to mice implanted with SGC-7901 cells. Jeong et al. investigated that anticancer effects of Saururus chinensis (Lour.) Baill (Saururaceae) ethanol extract against gastric cancer cell line AGS and NCI-N87 via caspase-dependent apoptosis through mitochondria membrane depolarization and activates JNK and p38 [80]. Kim et al. reported that Sophorae flavescentis Ait. Radix (Leguminosae/Fabaceae) inhibited proliferation and induced apoptosis of AGS cells by mitochondrial- and caspase-dependent apoptosis [81]. Activation of Fas and FasL, and caspase-3 were detected. Swertia mussotti Franch. (Gentianaceae) extract showed anti-proliferative effect and induced mitochondria-dependent apoptosis via depolymerization of cytoskeletal filaments, distrupted $\Delta \Psi \mathrm{m}$, and increased cytoplasmic levels of ROS [82]. Hosseini et al. studied the apoptotic ability of Cornus mas L. Bulbus (Cornaceae) extract against AGS cells in comparison with mouse fibroblast cell line, L929 [34]. Ghasemi et al. treated Urtia dioica radix (Urticaceae) hydroalchoholic extract on MKN-45 cell line (32, 125, $500 \mu \mathrm{g} / \mathrm{mL}$ for $24 \mathrm{~h})$ [35]. The extract induced apoptosis of tumor cells by inducing ROS formation and lipid peroxidation. Ngabire $e t$ al. reported that Aster incises Fisch. (Asteraceae) induced mitochondria-related apoptosis in AGS cells [85]. The expression levels of pro-apoptotic proteins such as Bak, BH3 interacting-domain death agonist (Bid), Bad, AIF, cytochrome c, cleaved caspases and cleaved PARP were significantly upregulated. Levels of anti-apoptotic proteins such as FLIP, Bcl-2 and Bcl-xL were lowered. Kustiawan et al. discovered that the methanol extract of Trigona incisa propolis represented cytotoxic activity to the human gastric cancer cell line, KATO-III [83]. Methanol extract of Schizandra chinensis Baill Fructus (Schisandraceae) was treated to AGS cells at the dose of 100, 200, 300, or $400 \mu \mathrm{g} / \mathrm{mL}$ for $24 \mathrm{~h}$ [84]. The extract induced apoptotic cell death and generated ROS via mitochondria- and caspase-dependent pathways, elevating Bax, caspase-3, and caspase-9 expressions and inhibiting Bcl-2 expression. It is suggested that c-Jun N-terminal kinase and p38 MAPK pathway is also involved. Study by Zhang et al. indicated that trichloromethane fractions of Incarvillea compacta Maxim. Radix (Bignoniaceae) inhibited the proliferation of EBV-positive AGS cells (AGS-EBV) by inducing EBV lytic replication and apoptosis [85]. Aqueous extract of Euphorbia esula Latex (Euphorbiaceae) promoted caspase dependent apoptosis regulated by Bax and Bcl-2 family [86]. 5, 10, 20, 40, 80, $160 \mathrm{mg} / \mathrm{L}$ extract was treated on SGC-7901 human gastric cancer cells for $24,48 \mathrm{~h}$. The activity of caspase- 8 and caspase-3 increased 1.73fold and 1.98-fold, respectively. Additionally, efficicicy of Astragalus membranceus Fisch. Radix (eguminosae/Fabaceae) was also studied in vivo model (Table 4) [79]. Xenograft nude mice injected SGC-7901 cells developed tumors of lesser size compared to mice only injected the vehicle. 
Table 3. Apoptosis-inducing natural product extracts in vitro.

\begin{tabular}{|c|c|c|c|c|c|c|}
\hline $\begin{array}{l}\text { Compound/ Ex- } \\
\text { tract }\end{array}$ & Source & $\begin{array}{l}\text { Experimental } \\
\text { Model }\end{array}$ & Dose; Duration & Efficacy & Mechanism & Reference \\
\hline Acetone extract & EL002332 & AGS & $5 \mu \mathrm{g} / \mathrm{mL} ; 24 \mathrm{~h}$ & Induction of apoptosis & $\begin{array}{c}\uparrow \text { Myc, caspase-3, -5, c-PARP } \\
\downarrow \text { Bcl-2 }\end{array}$ & [74] \\
\hline $\begin{array}{l}\text { Black currant ex- } \\
\text { tract }\end{array}$ & $\begin{array}{l}\text { (family: Saxifragaceae) } \\
\text { Ribes nigrum L. }\end{array}$ & MKN-45 & $\begin{array}{c}5,10,15,20,25 \mathrm{mg} / \mathrm{mL} \\
12,24,48 \mathrm{~h}\end{array}$ & Induction of apoptosis & $\begin{array}{c}\uparrow \text { Bax, caspase-8, JNK/p38, Fas, FasL } \\
\downarrow \Delta \Psi \mathrm{m}, \text { Bcl-2, p-Akt, p-ERK1/2 }\end{array}$ & {$[75]$} \\
\hline Ethanol extract & $\begin{array}{l}\text { (family: Ascomycota) } \\
\text { Cordyceps cicadae } \\
\text { (Miq.) Massee. }\end{array}$ & SGC-7901 & $\begin{array}{c}200,400,800 \mu \mathrm{g} / \mathrm{mL} ; 48 \\
\mathrm{~h}\end{array}$ & Induction of apoptosis & $\begin{array}{c}\uparrow \text { Bax, caspase-3, -6, -8, AIF, p53, Fas } \\
\downarrow \text { Bcl-2, PARP }\end{array}$ & [77] \\
\hline Ethanol extract & $\begin{array}{c}\text { (family: Smilacaceae) } \\
\text { Smilax glabra Roxb. Rhi- } \\
\text { zoma }\end{array}$ & SGC7901 & $10,20,30 \mu \mathrm{M} / \mathrm{L} ; 24 \mathrm{~h}$ & Induction of apoptosis & $\begin{array}{c}\uparrow \text { caspase- } 3, \text { Bax } \\
\downarrow \text { p-Akt, Bcl-2 }\end{array}$ & {$[78]$} \\
\hline Ethanol extract & $\begin{array}{c}\text { (family: Cornaceae) } \\
\text { Astragulus cornus mass } \\
\text { L. radix }\end{array}$ & SGC-7901 & $\begin{array}{c}25,50,100 \mu \mathrm{g} / \mathrm{mL} ; 3 \\
\text { days }\end{array}$ & Induction of apoptosis & $\begin{array}{c}\uparrow \text { Bax } \\
\downarrow \text { IL-6, STAT3 }\end{array}$ & [79] \\
\hline Ethanol extract & $\begin{array}{l}\text { (family: Saururaceae) } \\
\text { Saururus chinensis } \\
\text { (Lour.) Baill. }\end{array}$ & AGS, NCI-N87 & $\begin{array}{c}25,50 \mu \mathrm{g} / \mathrm{mL} ; 1,3,6,24 \\
30,48 \mathrm{~h}\end{array}$ & Induction of apoptosis & $\begin{array}{c}\uparrow \text { Bax, c-PARP, p-p38, p-JNK } \\
\downarrow \Delta \Psi \mathrm{m}, \mathrm{Bcl}-2\end{array}$ & {$[80]$} \\
\hline Ethanol extract & $\begin{array}{c}\text { (family: Legumi- } \\
\text { nosae/Fabaceae) Sopho- } \\
\text { rae flavescentis Ait. Ra- } \\
\text { dix }\end{array}$ & AGS & $\begin{array}{c}20,40,60,80,100,200 \\
\mu \mathrm{g} / \mathrm{mL} ; 24 \mathrm{~h}\end{array}$ & Induction of apoptosis & $\uparrow$ Fas, FasL, caspase-3 & {$[81]$} \\
\hline Ethanol extract & $\begin{array}{l}\text { (family: Gentianaceae) } \\
\text { Swertia mussotti Franch. }\end{array}$ & $\begin{array}{l}\text { MGC-803, BGC- } \\
823\end{array}$ & $\begin{array}{c}300,600,900 \mu \mathrm{g} / \mathrm{mL} ; 24 \\
\mathrm{~h}\end{array}$ & Induction of apoptosis & $\begin{array}{l}\uparrow \mathrm{ROS} \\
\downarrow \Delta \Psi \mathrm{m}\end{array}$ & {$[82]$} \\
\hline
\end{tabular}




\begin{tabular}{|c|c|c|c|c|c|c|}
\hline $\begin{array}{l}\text { Ethanol, water ex- } \\
\text { tract }\end{array}$ & $\begin{array}{l}\text { (family: Cornaceae) } \\
\text { Cornus mas L. Bulbus }\end{array}$ & AGS & $\begin{array}{l}\mathrm{IC}_{50} 5.44 \mathrm{mg} / \mathrm{mL} ; 48 \mathrm{~h} \\
\mathrm{IC}_{50} 2.44 \mathrm{mg} / \mathrm{mL} ; 72 \mathrm{~h}\end{array}$ & $\begin{array}{l}\text { Induction of apoptosis and } \\
\text { necrosis }\end{array}$ & & [34] \\
\hline $\begin{array}{l}\text { Hydroalchoholic } \\
\text { extract }\end{array}$ & $\begin{array}{l}\text { (family: Urticaceae) Ur- } \\
\text { tia dioica radix }\end{array}$ & MKN-45 & $32,125,500 \mu \mathrm{g} / \mathrm{mL} ; 24 \mathrm{~h}$ & Induction of apoptosis & $\uparrow \operatorname{ROS}$ & [35] \\
\hline Methanol extract & $\begin{array}{l}\text { (family: Asteraceae) As- } \\
\quad \text { ter incises Fisch. * }\end{array}$ & AGS & $80,100,140 \mu \mathrm{g} / \mathrm{mL} ; 24 \mathrm{~h}$ & Induction of apoptosis & $\begin{array}{c}\uparrow \text { Bid, Bad, Bak, cyt c, c-caspase- } 3,-8,- \\
9, \text { AIF, ADP-ribose, PARP } \\
\downarrow \text { Bcl-2, Bcl-xL }\end{array}$ & [87] \\
\hline Methanol extract & $\begin{array}{l}\text { Propolis produced by } \\
\text { Trigona incisa }\end{array}$ & KATO-III & $\begin{array}{c}\mathrm{IC}_{50} 6.06 \pm 0.39 \mu \mathrm{g} / \mathrm{mL} \\
48 \mathrm{~h}\end{array}$ & Induction of cytotoxicity & & [83] \\
\hline Methanol extract & $\begin{array}{c}\text { (family: Schisan- } \\
\text { draceae) Schizandra } \\
\text { chinensis Baill Fructus }\end{array}$ & AGS & $\begin{array}{l}100,200,300,400 \\
\mu \mathrm{g} / \mathrm{mL} ; 24 \mathrm{~h}\end{array}$ & Induction of apoptosis & $\begin{array}{c}\uparrow \mathrm{ROS}, \text { Bax, caspase-3, }-9 \\
\downarrow \text { Bcl-2 }\end{array}$ & [84] \\
\hline $\begin{array}{l}\text { Trichloromethane } \\
\text { fractions / Ethanol } \\
\text { extract }\end{array}$ & $\begin{array}{l}\text { (family: Bignoniaceae) } \\
\text { Incarvillea compacta } \\
\quad \text { Maxim. Radix }\end{array}$ & AGS-EBV & $5,10,20 \mu \mathrm{g} / \mathrm{mL} ; 24 \mathrm{~h}$ & $\begin{array}{l}\text { Induction of lytic cytotoxi- } \\
\text { city and apoptosis }\end{array}$ & $\uparrow \mathrm{Bax} \downarrow$ Bcl-2 & [85] \\
\hline Water extract & $\begin{array}{l}\text { (family: Euphorbiaceae) } \\
\text { Euphorbia esula Latex }\end{array}$ & SGC-7901 & $\begin{array}{c}5,10,20,40,80,160 \\
\mathrm{mg} / \mathrm{L} ; 24,48 \mathrm{~h}\end{array}$ & induction of apoptosis & $\begin{array}{c}\uparrow \text { Bax, caspase- } 3 \text {, caspase-8 } \\
\downarrow \text { Bcl-2 }\end{array}$ & [86] \\
\hline
\end{tabular}

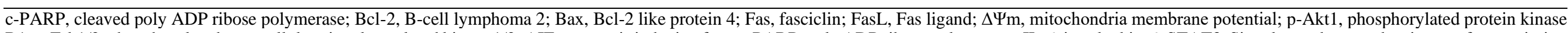

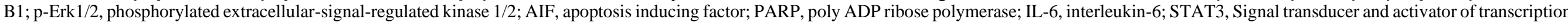

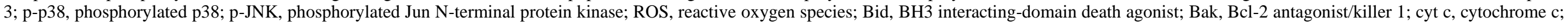
Bcl-xL, B-cell lymphoma-extra large.*The part of the plant used in this was not mentioned in the study. 
Table 4. Apoptosis-inducing natural product extracts in vivo.

\begin{tabular}{|c|c|c|c|c|c|c|}
\hline $\begin{array}{c}\text { Compound/ Ex- } \\
\text { tract }\end{array}$ & Source & $\begin{array}{c}\text { Experimental } \\
\text { Model }\end{array}$ & Dose; Duration & Efficacy & Mechanism & Reference \\
\hline Ethanol extract & $\begin{array}{c}\text { (family: Legumi- } \\
\text { nosae/Fabaceae) Astrag- } \\
\text { alus membranceus Fisch. } \\
\text { Radix }\end{array}$ & $\begin{array}{c}\text { nude mice / SGC- } \\
7901\end{array}$ & $\begin{array}{c}60,120 \mathrm{mg} / \mathrm{kg} \text {; daily, for } \\
3 \text { weeks }\end{array}$ & Inhibition of tumor growth & $\downarrow$ VEGF, IL-6 & [79] \\
\hline
\end{tabular}

VEGF, Vascular endothelial growth factor; IL-6, interleukin-6

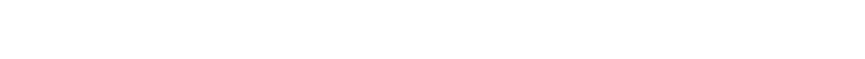

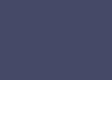 \\ Preprints (Www.preprints.org) | NOT PEER-REVIEWED | Posted: 27 July 2021}




\subsubsection{Apoptosis-inducing mixtures in gastric cancer}

Four studies discussing decoctions or pills of TCM focused on the apoptosis-inducing abilities of the mixtures (Table 5,6). Jinlong capsule (JLC) is an effective Traditional Chinese Medicine widely used to treat gastric cancer patients [88]. It was discovered that JLC induces mitochondrial apoptosis of MGC-803 and BGC-823 cells through a cascadedependent pathway [89]. Xu et al. demonstrated that the TCM decoction Xiao Tan He Wei induces apoptosis against gastric tumors [90]. 1-Methyl-3-nitro-1-nitrosoguanidine (MNNG) was treated to wistar rats and GES-1 cells to induce precancerous lesions of gastric carcinoma in vivo and in vitro. The decoction induced apoptosis of GES-1 cells by inhibiting the NF- $\mathrm{KB}$ pathway and showed anti-metastatic efficacy to wistar rats via cell cycle arrest. Yangzhen Sanjie decoction is made from the medicinal herbs Astragalus membranceus Fisch. Radix (Leguminosae/Fabaceae), Scutellariae barbata D. Don Herba (Lamiaceae), Arisaematis preparatum Preparatum, Citri sarcodactylis Fructus Swingle. (Rutaceae), Cremastrae pseudobulbus seu Pleiones. (Orchidaceae), Curcuma longa L. Rhizoma (Zingiberaceae) [91]. The decoction was diluted into a $10 \%$ serum and treated to human gastric carcinoma AGS and HS-746T cells for $48 \mathrm{~h}$. The treatment enhanced Let-7a miRNA expression, which leads to the downregulation of c-Myc and consequent promotion of apoptosis in both cell lines. Expression of other microRNAs of the let-7 family were altered as well. So-CheungRyong-Tang consists of 8 herbs: Ephedra sinica Stapf. Radix Ephedraceae), Schisandra chinensis Fructus (Turcz.) Baill. (Schisandraceae), Paeonia lactiflora pall. Radix (Paeoniaceae), Pinellia ternate (Thunb.) Breit rhizome (Araceae), Cinnamomum cassia Presl radix (Lauraceae), Zingiber officinale Rosc radix (Zingiberaceae), Asiasarum heterotropoides rhizome (Aistolochiaceae), Glycyrrhiza glabra Fisch. Radix (Fabaceae) Yim et al. reported that So-Cheung-Ryong-Tang showed greater apoptotic ability against AGS and NUGC-3 cells when fermented [92]. In an in vivo approach, tumor weight of the athymic nu/nu mice were subcutaneously injected HCT116 cells was smallest in the group treated with the fermented decoction (Table 6). 
Table 5. Apoptosis-inducing mixtures in vitro.

\begin{tabular}{|c|c|c|c|c|c|c|}
\hline $\begin{array}{c}\text { Compound/ } \\
\text { Extract }\end{array}$ & Source & $\begin{array}{c}\text { Experimental } \\
\text { Model }\end{array}$ & Dose; Duration & Efficacy & Mechanism & References \\
\hline Jinlong Capsule & Bungarus, Agkistrodon, Gecko & $\begin{array}{c}\text { MGC-803, } \\
\text { BGC-823 }\end{array}$ & $\begin{array}{c}0,10.2,0.4,0.8 \\
\mathrm{mg} / \mathrm{mL} ; 24 \mathrm{~h}\end{array}$ & $\begin{array}{l}\text { Induction of } \\
\text { apoptosis }\end{array}$ & $\begin{array}{l}\uparrow \text { Bax, caspase-3 } \\
\downarrow \text { Bcl-2, survivin }\end{array}$ & [89] \\
\hline $\begin{array}{c}\text { Xiao Tan } \mathrm{He} \\
\text { Wei decoction }\end{array}$ & $\begin{array}{l}\text { (family: Umbelliferae) Bupleurum falcatum Linne. Radix, (family: } \\
\text { Araceae) Pinellia ternata (Thunb.) Makino Rhizoma, (family: Polypo- } \\
\text { raceae) Poria cocos Wolf., (family: Ranunculaceae) Coptis chinensis } \\
\text { Franch., (family: Rubiaceae) Oldenlandia diffusa (Willd.) Roxb. } \\
\text { Herba, (family: Compositae) Taraxaci Herba*, (family: Lauraceae) } \\
\text { Cinnamonun cassia J.Presl twig, (family: Polygonaceae) Rhubarb*, } \\
\text { (family: Paeoniaceae) Paeonia lactiflora Pallas., (family: Legumi- } \\
\text { nosae/Fabaceae) Glycyrrhizae uralensis Fischer. }\end{array}$ & GES-1 & $\begin{array}{l}0.01,0.02,0.04 \\
0.08,0.16,0.32 \\
0.64,1.28,2.56 \\
\mathrm{~g} / \mathrm{L} ; 24,48,72 \\
\quad 96 \mathrm{~h}\end{array}$ & $\begin{array}{l}\text { Induction of } \\
\text { apoptosis }\end{array}$ & $\begin{array}{l}\uparrow \text { Bax, c-caspase- } \\
3, \text { I } \mathrm{B} \\
\downarrow \mathrm{Bcl}-2, \mathrm{p} 65, \mathrm{NF}- \\
\mathrm{\kappa B}\end{array}$ & [90] \\
\hline $\begin{array}{c}\text { Xiao Tan He } \\
\text { Wei decoction }\end{array}$ & $\begin{array}{l}\text { (family: Umbelliferae) Bupleurum falcatum Linne. Radix, (family: } \\
\text { Araceae) Pinellia ternata (Thunb.) Makino Rhizoma, (family: Polypo- } \\
\text { raceae) Poria cocos Wolf., (family: Ranunculaceae) Coptis chinensis } \\
\text { Franch., (family: Rubiaceae) Oldenlandia diffusa (Willd.) Roxb. } \\
\text { Herba, (family: Compositae) Taraxaci Herba*, (family: Lauraceae) } \\
\text { Cinnamonun cassia J.Presl twig, (family: Polygonaceae) Rhubarb*, } \\
\text { (family: Paeoniaceae) Paeonia lactiflora Pallas., (family: Legumi- } \\
\text { nosae/Fabaceae) Glycyrrhizae uralensis Fischer. }\end{array}$ & Wistar rats & $\begin{array}{l}3.4 \mathrm{~mL} / \mathrm{kg} ; 2 \\
\text { weeks }\end{array}$ & $\begin{array}{c}\text { Inhibition of } \\
\text { metastasis }\end{array}$ & $\begin{array}{l}\uparrow \text { Bax, c-caspase- } \\
3, \text { I } \mathrm{B} \\
\downarrow \mathrm{Bcl}-2, \mathrm{p} 65, \mathrm{NF}- \\
\mathrm{\kappa B}\end{array}$ & [90] \\
\hline $\begin{array}{c}\text { Yangzhen San- } \\
\text { jie } \\
\text { decoction }\end{array}$ & $\begin{array}{l}\text { (family: Leguminosae/Fabaceae) Astragalus membranceus Fisch. Ra- } \\
\text { dix, (family: Lamiaceae) Scutellariae barbata D.Don Herba, } \\
\text { Arisaematis preparatum Citri sarcodactylis Fructus, Cremastrae Tu- } \\
\text { ber*, (family: Zingiberaceae) Curcuma longa L. Rhizoma }\end{array}$ & $\begin{array}{l}\text { (1) AGS } \\
\text { (2) HS-746T }\end{array}$ & $10 \%$ serum; $48 \mathrm{~h}$ & $\begin{array}{l}\text { Induction of } \\
\text { apoptosis }\end{array}$ & $\uparrow$ let-7a $\downarrow$ c-Myc & [91] \\
\hline $\begin{array}{c}\text { Fermented So- } \\
\text { Cheong-Ryong- } \\
\text { Tang }\end{array}$ & $\begin{array}{l}\text { (family: Ephedraceae) Ephedra sinica Stapf. Radix, (family: Schisan- } \\
\text { draceae) Schisandra chinensis Fructus (Turcz.) Baill., (family: Paeo- } \\
\text { niaceae) Paeonia lactiflora pall. Radix, (family: Araceae) Pinellia } \\
\text { ternate (Thunb.) Breit Rhizoma, (family: Lauraceae) Cinnamomum } \\
\text { cassia Presl radix, (family: Zingiberaceae) Zingiber officinale Rosc } \\
\text { radix, (family: Aistolochiaceae) Asiasarum heterotropoides Rhizoma, } \\
\text { (family: Fabaceae) Glycyrrhiza glabra Fisch. Radix }\end{array}$ & AGS, NUGC-3 & $\begin{array}{l}500 \mu \mathrm{g} / \mathrm{mL}: 0.5 \\
1,3,6 \mathrm{~h}\end{array}$ & $\begin{array}{l}\text { Induction of } \\
\text { apoptosis }\end{array}$ & $\begin{array}{l}\uparrow \text { caspase- } 3,-8,-9, \\
\text { c-PARP, cyclin } \\
\text { D1, cyclin E1, cy- } \\
\text { clin B1, p21 } \\
\downarrow \text { p-p38, p-ERK, } \\
\quad \text { p-JNK }\end{array}$ & [92] \\
\hline
\end{tabular}

c-PARP, cleaved poly ADP ribose polymerase; p-p38, phosphorylated p38; p-Erk, phosphorylated extracellular-signal-regulated kinase; p-JNK, phosphorylated Jun N-terminal protein kinase; Bax, Bcl-2 like protein 4; Bcl-2, B-cell lymphoma 2; $\Delta \Psi \mathrm{m}$, mitochondria membrane potential; c-Myc, cellular MYC proto-oncogene protein; IKB, inhibitor of nuclear factor kappa. *Taraxaci Herba includes Taraxacum platycarpum H. Dahlstedt, Taraxacum officinale Weber, Taraxacum mongolicum Handel-Mazzetti, and Taraxacum coreanum Nakai. Which species was used was not indicated in the study. *Rhubarb includes Rheum palmatum Linné Radix et Rhizoma, Rheum tanguticum Maximowicz ex Balf. And Rheum officinale Baillon. Which species was used was not indicated in the study. ${ }^{*}$ Chremastrae Tuber includes Cremastra appendiculata (D.Don) Makino, Pleione bulbocodioides Rolfe, and Pleione yunnanensis Rolfe. The species used in the study was not indicated. 
Table 6. Apoptosis-inducing mixtures in vivo

\begin{tabular}{|c|c|c|c|c|c|c|}
\hline $\begin{array}{c}\text { Compound } / \text { Ex- } \\
\text { tract }\end{array}$ & Source & $\begin{array}{l}\text { Experi- } \\
\text { mental } \\
\text { Model }\end{array}$ & $\begin{array}{l}\text { Dose; Dura- } \\
\text { tion }\end{array}$ & Efficacy & Mechanism & Reference \\
\hline $\begin{array}{l}\text { Fermented So- } \\
\text { Cheong-Ryong- } \\
\text { Tang }\end{array}$ & $\begin{array}{l}\text { (family: Ephedraceae) Ephedra sinica Stapf. Radix, } \\
\text { (family: Schisandraceae) Schisandra chinensis Fructus } \\
\text { (Turcz.) Baill., (family: Paeoniaceae) Paeonia lacti- } \\
\text { flora pall. Radix, (family: Araceae) Pinellia ternate } \\
\text { (Thunb.) Breit rhizoma, (family: Lauraceae) Cin- } \\
\text { namomum cassia Presl radix, (family: Zingiberaceae) } \\
\text { Zingiber officinale Rosc radix, (family: Aistolochia- } \\
\text { ceae) Asiasarum heterotropoides rhizoma, (family: Fa- } \\
\text { baceae) Glycyrrhiza glabra Fisch. Radix }\end{array}$ & $\begin{array}{c}\text { athymic } \\
\text { nu/nu mice / } \\
\text { HCT116 }\end{array}$ & $\begin{array}{c}157.5 \mathrm{mg} / \mathrm{kg} ; \\
14 \text { days }\end{array}$ & $\begin{array}{l}\text { Inhibition of tu- } \\
\text { mor growth }\end{array}$ & & [92] \\
\hline
\end{tabular}




\subsection{Emergeing role of autophagy in gastric cancer treatment mediated by natural products}

Autophagy is a cellular process in which cytoplasmic contents are degraded within the lysosome/vacuole, and the resulting constituents are recycled [93, 94]. Autophagy can be classified into macroautophagy, microautophagy, and chaperone-mediated autophagy (CMA) [95]. Among these, macroautophagy, which has been studied the most, is the process of forming autophagosomes that surround organelles and fuses with lysosomes and natural products modulate autophagy [96]. In addition, autophagy can also be categorized into selective and nonselective autophagy. Based on the isolation target, separate kinds of selective autophagy such as mitophagy, pexophagy, and xenophagy can be distinguished [97]. Macroautophagy consists of several sequential steps: initiation, nucleation, elongation, maturation and fusion with the lysosome [98]. Phagosome originates from omegasome, a subdomain of ER, and associates with other organelles such as mitochondria, golgi complex, plasma membrane, recycling endosome, etc. during its development. Four molecules, Unc-51-like kinase 1/2 (ULK1/2), autophagy-related gene 13 (ATG13), family 200-kD interacting protein (FIP200) and AtG101 form the ULK1/2 complex and initiates the process. The mechanistic target of rapamycin complex 1 (mTORC1) is a major inhibitor of the ULK1/2 complex [93]. AMP-activated protein kinase (AMPK) inhibits mTORC1 and leads to the activation of ULK1/2 complex [98]. The ULK1/2 complex phosphorylates the class III phosphatidylinositol-3-kinase (PI3K) vacuole protein sorting 34 (VPS34) complex consisting of VPS15, Beclin-1 and AtG14 complex, which promotes the formation of phosphatidylinositol-3-phosphate (PI3P), which is an essential lipid molecule required for the nucleation step of the phagophore [99]. PI3P recruits PI3P-binding proteins such as WIPI2B, a protein known to facilitate LC3-lipidation [100]. Formation and expansion of the phagophore is related to the generation of Atg12-5-16L1 complex. Atg12 binds with Atg5 and composes a complex with Atg16L. The Atg12-5-16L1 complex lipidates LC3-I into LC3-II [101, 102]. LC3-II, considered a marker of autophagy, is essential for phagosome elongation and fusion $[103,104]$. When the phagosome encloses and becomes a mature autophagosome, it fuses with a lysosome and degradation and recycling process follows (Figure 2). 


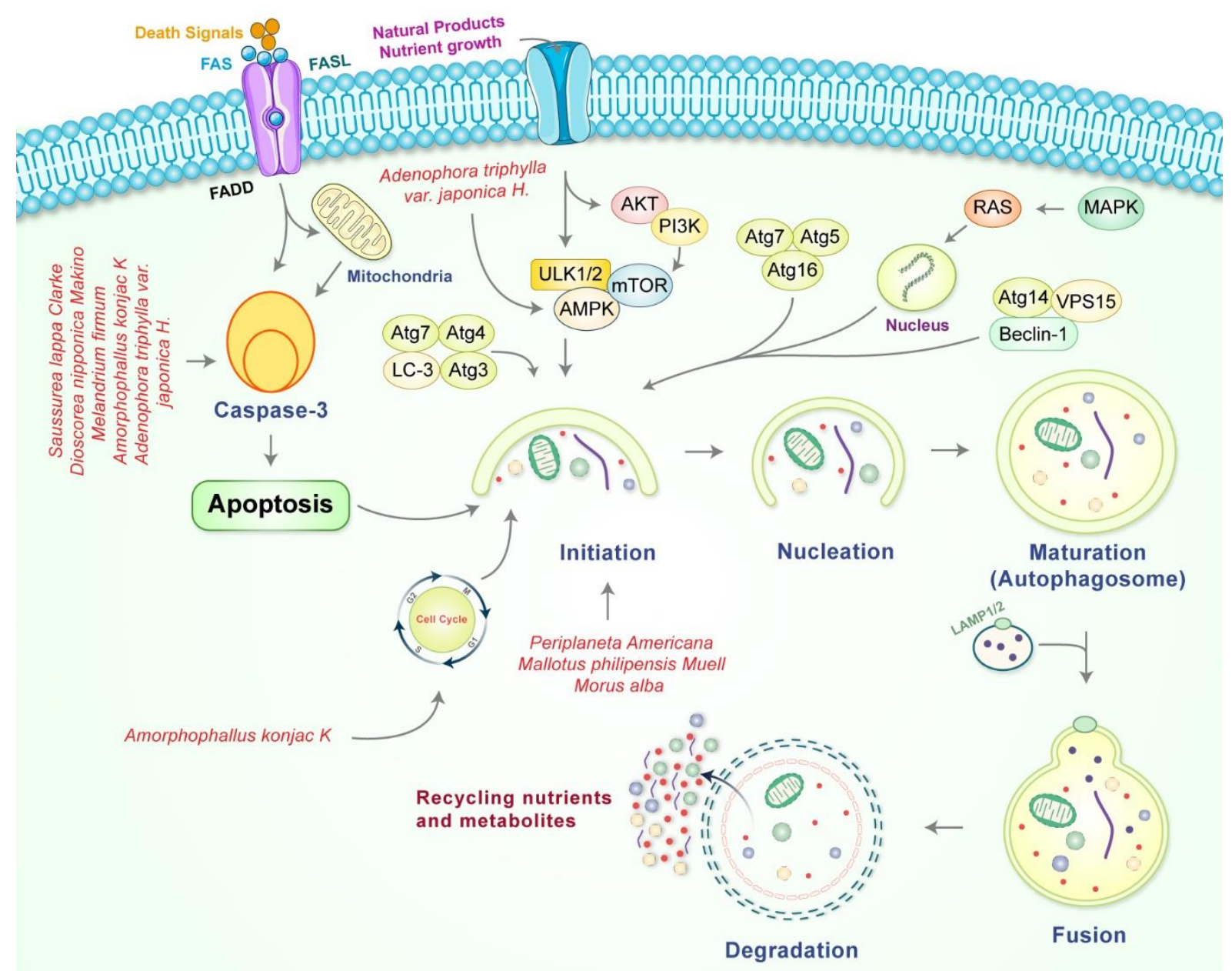

Figure 2. Natural products regulates molecular mechanism of autophagy. Natural products initiate autophagy by the formation of a pre-autophagosomal structure via association of PI3K-AMPK, mammalian target of rapamycin (mTOR), ULK1, Vps34, and the Beclin-1 complex which contribute to the formation of the pre-autophagosomal structure in addition to activate phagophore formation. Fusion of mature autophagosome as well as lysosome causes autolysosome formation. Lastly, elimination of molecule is happened by acid hydrolases which has been produced nutrients as well as recycling metabolites.

Two single compounds and two plant extracts were reported to induce autophagy along with apoptosis against gastric cancer cells (Table 7). Rottlerin, extracted from Mallotus philipensis Muell (Euphorbiaceae), induced autophagy and caspase independent apoptosis against SGC-7901 and MGC-803 cells [105]. Autophagy is induced by downregulating mTOR and S-phase kinase-associated protein 2 (Skp2). 3-O- $\beta$-D-galactopyranosyl-( $1 \rightarrow 2)$-[ $\beta$-D-xylopyranosyl- $(1 \rightarrow 3)]-\beta$-D-glucuronopyranosyl-28-O-[ $\alpha$-L-arabinopyranosyl-( $(\rightarrow 4)$ - $\alpha$-L-arabinopyranosyl-( $(\rightarrow 3)-\beta$-D-xylopyranosyl-( $1 \rightarrow 4)-\alpha$-L-rhamnopyranosyl-( $(1 \rightarrow 2)-\beta$-D-fucopyranosyl] quillaic acid is an oleanane-type triterpenoid saponin extracted from Adenophora triphylla var. japonica H. (Campanulaceae) [106]. Morus alba root extract, contain oxyresveratrol, has been found to accumulate ROS production and initiated autophagic and apoptotic cell death via FOXO-caspase-3 pathway $[107,108]$. It has been demonstrated that cytotoxic activity on AGS, MKN-45 and KATO-III human gastric cancer cells via induction of caspases activation and autophagy via Akt/NF- $\kappa B$ pathway in AGS cells. Chen et al. demonstrated the anti-cancer activity of the ethanol and ligarine based extract of the tuber of Amorphophallus konjac K. Koch Tuber (Araceae) against SGC7901 and AGS cells (IC50 of 35-45 $\mu \mathrm{g} / \mathrm{mL}$ ) [109]. Flux analysis of autophagy and increase of the level of LC3-II revealed induction of autophagy by the tuber of A. konjac. G0/G1 phase cell cycle arrest has detected by flow cytometry. Chen et al. determined apoptosis- and autophagy-inducing effects of kangfuxin, an organic extract of Periplaneta 
americana Linnaeus. (Blattidae), against SGC-7901 cell line [110]. Proteins that mediate ER stress mediated apoptosis including glucose-regulated protein 78 (GRP78), C/EBP-homologous protein (CHOP) and caspase-12 has been greatly upregulated in the group treated kangfuxin. Transmission electron microscopy (TEM) image results has been shown much more autophagosomes compared with the control. In addition, the LC3-I/LC3-II ratio and expression levels of Beclin-1 were also higher in the kanfuxin group. Additionally, Natural plant extracts of Saussurea lappa Clarke, Dioscorea nipponica Makino, and Melandrium firmum has been found to induce anti-proliferative as well as apoptotic function [111-113]. Therefore, autophagy induction by natural products might possibly be targeted as a potential therapeutic approach to control gastric cancer. 
Table 7. Autophagy-Inducing Natural Products in vitro.

\begin{tabular}{|c|c|c|c|c|c|c|}
\hline Classification & Compound/Extract & Source & Experimental Model & $\begin{array}{l}\text { Dose; Du- } \\
\text { ration }\end{array}$ & Mechanism & References \\
\hline Polyphenol & Rottlerin & $\begin{array}{l}\text { (family: Euphorbia- } \\
\text { ceae) } \\
\text { Mallotus philipensis } \\
\text { Muell. }\end{array}$ & SGC-7901, MGC-803 & $\begin{array}{c}2,4,8 \mu \mathrm{M} \\
24 \mathrm{~h}\end{array}$ & $\begin{array}{c}\uparrow \mathrm{LC} 3-\mathrm{II} \\
\downarrow \mathrm{mTOR}, \text { Skp2 }\end{array}$ & [105] \\
\hline \multirow[t]{3}{*}{ Terpenoid } & $\begin{array}{c}\text { 3-O- } \beta \text {-D-galactopyranosyl-( }(1 \rightarrow 2) \text {-[ } \beta \text {-D- } \\
\text { xylopyranosyl- }(1 \rightarrow 3)]-\beta \text {-D-glucuronopyra- } \\
\text { nosyl-28-O-[ } \alpha \text {-L-arabinopyranosyl- }(1 \rightarrow 4)- \\
\alpha \text {-L-arabinopyranosyl- }(1 \rightarrow 3)-\beta \text {-D-xylopyra- } \\
\text { nosyl- }(1 \rightarrow 4)-\alpha \text {-L-rhamnopyranosyl- }(1 \rightarrow 2) \text { - } \\
\beta \text {-D-fucopyranosyl] quillaic acid }\end{array}$ & $\begin{array}{l}\text { (family: Campanu- } \\
\text { laceae) } \\
\text { Adenophora triphylla } \\
\text { var. japonica } \mathrm{H} .\end{array}$ & $\begin{array}{c}\text { AGS, MKN-45, KATO } \\
\text { III }\end{array}$ & $\begin{array}{l}10,20,30 \\
\mu \mathrm{M} ; 24 \mathrm{~h}\end{array}$ & $\begin{array}{c}\uparrow p \text {-JNK, p-p38, p-AMPK, Bax, cyt c, } \\
\text { caspase-3, c-PARP1, LC3-II } \\
\downarrow \text { p-ERK, p-Akt, p-mTOR, NF-kB, } \\
\text { COX-2, Cyclin } D_{1} \text {, VEGF, Bcl-2, Bid }\end{array}$ & {$[106]$} \\
\hline & Organic extract & $\begin{array}{c}\text { (family: Araceae) } \\
\text { Amorphophallus } \\
\text { konjac K. Koch Tuber }\end{array}$ & SGC-7901, AGS & $\begin{array}{c}50 \mu \mathrm{g} / \mathrm{mL} \\
24 \mathrm{~h}\end{array}$ & $\begin{array}{c}\uparrow \text { Bax, c-caspase-9, LC3-II } \\
\downarrow \text { survivin, Bcl-2 }\end{array}$ & [109] \\
\hline & $\begin{array}{l}\text { Organic extract } \\
\text { (kangfuxin) }\end{array}$ & $\begin{array}{l}\text { (family: Blattidae) } \\
\text { Periplaneta ameri- } \\
\text { cana } \text { Linnaeus. }\end{array}$ & SGC-7901 & $\begin{array}{c}0.1,1 \\
\mu \mathrm{g} / \mathrm{mL} \\
48 \mathrm{~h}\end{array}$ & $\begin{array}{c}\text { 个GRP78, CHOP, caspase-12, LC3- } \\
\text { II/LC3-I, Bax } \\
\downarrow \text { Bcl-2 }\end{array}$ & [110] \\
\hline
\end{tabular}

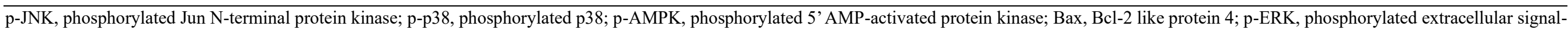

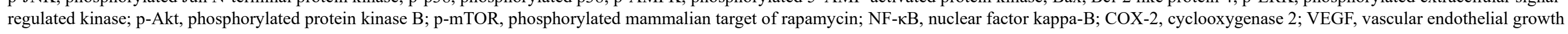
factor; Bcl-2, B-cell lymphoma 2; Skp2, S-phase kinase-associated protein 2; GRP78, glucose-regulated protein 78; CHOP, C/EBP-homologous protein. 


\subsection{Role of natural products to arrest cell cycle in gastric cancer}

The cell cycle is regulated through a series of control systems that in turn promote or inhibit cell division. Programmed cell death and cell cycle regulation occur together in many cancerous cells since the tumor suppressor gene $p 53$ and downstream proteins regulate both events [114]. A variety of natural substances were described as causing cell death and inhibited cell proliferation by seizing the cell cycle according to the phase of cell cycle arrest (Table 8). The studies performed cell cycle analysis through flow cytometry to observe cell cycle distribution, albeit the proteins responsible for the event were not confirmed in a large portion of the investigations. Berberine, TTF, ginsenoside- $\mathrm{Rh} 2$, crosolic acid, rottlerin, grifolin, methanol extract of aster incises (Asteraceae), organic extract of Amorphophallus konjac K.Koch Tuber (Araceae), and xiao tan he wei decoction induced G0/G1 phase cell cycle arrest [38, 45, 49, 58, 62, 87, 90, 105, 109]. Toosendanin increased the proportion of cells in the G1 and S phase $[67,68]$. cyclovirobuxine D, GFG-3a, ethanol extract of Cordyceps cicadae Massee. (Ascomycota) and Swertia mussotti Franch. (Gentianaceae) induced S phase cell cycle arrest [39, 40, 77, 82]. Deacetylisovaltratum, Euphorbia lunulate (Euphorbiaceae) extract, phloretin, trifolirhizin, and tanshinone IIA triggered G2/M phase cell cycle arrest $[44,46,55,60,76]$. Jinlong Capsule halted the cell cycle in S and $\mathrm{G} 2 / \mathrm{M}$ phase [89]. C. cicadae extract was reported to decrease concentrations of cyclin E/CDK2 complex and cyclin A/CDK2 complex [77]. Tanshinone-IIA inhibited the expressions of CDC2, cyclin A and cyclin B1 [46]. 
Table 8. Cell Cycle Arrest-Inducing Natural Products.

\begin{tabular}{|c|c|c|c|c|c|c|c|}
\hline $\begin{array}{l}\text { Phase of cell } \\
\text { cycle arrest }\end{array}$ & Classification & $\begin{array}{l}\text { Compound/ } \\
\text { Extract }\end{array}$ & Source & $\begin{array}{l}\text { Experimental } \\
\text { Model }\end{array}$ & $\begin{array}{l}\text { Dose; Du- } \\
\text { ration }\end{array}$ & Mechanism & References \\
\hline $\mathrm{G} 0 / \mathrm{G} 1$ & Alkaloids & Berberine & $\begin{array}{c}\text { (family: Ranunculaceae) } \\
\text { Coptidis japonica Makino Rhizoma }\end{array}$ & SGC-7901 & $\begin{array}{c}5,10,20 \\
\mu \mathrm{M} ; 24,48 \\
\mathrm{~h}\end{array}$ & & [38] \\
\hline G0/G1 & Polyphenols & $\begin{array}{c}\text { 6, 7, 30-tri- } \\
\text { methoxy-3, } 5 \\
40 \\
\text {-trihydroxy fla- } \\
\text { vone (TTF) }\end{array}$ & $\begin{array}{c}\text { (family: Saxifragaceae) } \\
\text { Chrysosplenium nudicaule Ledeb Herba }\end{array}$ & SGC-7901 & $\begin{array}{l}2,4,8,16 \\
32 \mu \mathrm{g} / \mathrm{mL} \\
24,48,72 \mathrm{~h}\end{array}$ & $\begin{array}{l}\uparrow \text { endogenous } \mathrm{Ca} 2+ \\
/ \mathrm{Mg}^{2+} \text { dependent en- } \\
\text { donuclease }\end{array}$ & [49] \\
\hline $\mathrm{G} 0 / \mathrm{G} 1$ & Phytosterols & $\begin{array}{l}\text { Ginsenoside - } \\
\text { rh2 }\end{array}$ & $\begin{array}{c}\text { (family: Araliaceae) Panax ginseng C.A. } \\
\text { Mey Radix }\end{array}$ & SGC-7901 & $\begin{array}{c}5,10,20 \\
\mu \mathrm{g} / \mathrm{mL} ; 24 \\
48 \mathrm{~h}\end{array}$ & $\begin{array}{l}\uparrow \mathrm{Bax} \\
\downarrow \mathrm{Bcl}-2\end{array}$ & [45] \\
\hline G0/G1 & Terpenoids & Crosolic acid & $\begin{array}{c}\text { (family: Actinidiaceae) } \\
\text { Actinidia valvata Dunn Radix }\end{array}$ & BGC-823 & $\begin{array}{l}20,40,80 \\
\mu \mathrm{g} / \mathrm{mL} ; 72 \\
\mathrm{~h}\end{array}$ & $\begin{array}{l}\uparrow \mathrm{Bax}, \mathrm{smac}, \mathrm{I \kappa B} \alpha \\
\downarrow \mathrm{Fas}, \mathrm{Bcl}-2, \mathrm{p} 65, \mathrm{p}- \\
\text { I } \mathrm{K} \mathrm{B} \alpha, \mathrm{NF}-\kappa \mathrm{B}\end{array}$ & {$[58]$} \\
\hline G1 & Terpenoids & Grifolin & $\begin{array}{l}\text { (family: Albatrellaceae) } \\
\text { Albatrellus confluens (Alb. \& Schwein.) } \\
\text { Kotl. \& Pouzar }\end{array}$ & $\begin{array}{l}\text { BGC-823, SGC- } \\
7901\end{array}$ & $\begin{array}{l}10,50 \mu \mathrm{M} \\
48 \mathrm{~h}\end{array}$ & $\begin{array}{l}\uparrow \text { caspase- } 9,-3, \\
\text { CDKN2 } \\
\downarrow \text { MEK1, MEKK3 } \\
\text { MEK5 }\end{array}$ & [62] \\
\hline G1 & & $\begin{array}{l}\text { Methanol ex- } \\
\text { tract }\end{array}$ & (family: Asteraceae) Aster incises Fisch. * & AGS & $\begin{array}{l}80,100 \\
140 \mu \mathrm{g} / \mathrm{mL} \\
24 \mathrm{~h}\end{array}$ & $\begin{array}{c}\uparrow \text { Bid, Bad, Bak, cyt c, } \\
\text { c-caspase- } 3,-8,-9, \\
\text { AIF, ADP-ribose, } \\
\text { PARP } \\
\downarrow \text { Bcl-2, Bcl-xL }\end{array}$ & [87] \\
\hline
\end{tabular}




\begin{tabular}{|c|c|c|c|c|c|c|c|}
\hline G1 & & Organic extract & $\begin{array}{c}\text { (family: Araceae) } \\
\text { Amorphophallus konjac K. Koch Tuber }\end{array}$ & SGC-7901, AGS & $\begin{array}{c}50 \mu \mathrm{g} / \mathrm{mL} \\
24 \mathrm{~h}\end{array}$ & $\begin{array}{c}\uparrow \text { Bax, c-caspase-9, } \\
\text { LC3-II } \\
\downarrow \text { survivin, Bcl-2 }\end{array}$ & [109] \\
\hline $\mathrm{S}$ & Alkaloids & $\begin{array}{l}\text { Cyclovirobux- } \\
\text { ine D }\end{array}$ & $\begin{array}{c}\text { (family: Buxaceae) } \\
\text { Buxus microphylla } \text { Richardii Radix }\end{array}$ & $\begin{array}{l}\text { MGC-803, } \\
\text { MKN-28 }\end{array}$ & $\begin{array}{l}30,60,120 \\
\mu \mathrm{M} / \mathrm{L} ; 48 \mathrm{~h}\end{array}$ & $\begin{array}{c}\uparrow \mathrm{c} \text {-caspase-3, Bax } \\
\downarrow \mathrm{Bcl}-2\end{array}$ & [39] \\
\hline $\mathrm{S}$ & Alkaloids & GFG-3a & $\begin{array}{l}\text { (family: Meripilaceae) } \\
\text { Grifola frondose (Diks.) Gray Mycelia }\end{array}$ & SGC-7901 & $\begin{array}{l}100,200 \\
\mu \mathrm{g} / \mathrm{mL} ; 24 \\
48 \mathrm{~h}\end{array}$ & $\begin{array}{c}\uparrow \text { RBBP4, caspase-3, - } \\
\text { 8, p53, Bax, Bad } \\
\downarrow \text { RUVBL, NPM, Bcl- } \\
\text { 2, Bcl-xL, PI3K, Akt1 }\end{array}$ & [40] \\
\hline $\mathrm{S}$ & & Ethanol extract & $\begin{array}{c}\text { (family: Ascomycota) Cordyceps cicadae } \\
\text { (Miq.) Massee. }\end{array}$ & SGC-7901 & $\begin{array}{l}200,400 \\
800 \mu \mathrm{g} / \mathrm{mL} \\
\quad 48 \mathrm{~h}\end{array}$ & $\begin{array}{c}\uparrow \text { Bax, caspase-3, }-6,- \\
\text { 8, AIF, p53, Fas } \\
\downarrow \text { Bcl-2, PARP }\end{array}$ & [77] \\
\hline
\end{tabular}




\begin{tabular}{|c|c|c|c|c|c|c|c|}
\hline $\mathrm{S}$ & & Ethanol extract & $\begin{array}{c}\text { (family: Gentianaceae) Swertia mussotti } \\
\text { Franch. }\end{array}$ & $\begin{array}{l}\text { MGC- } 803 \text {, BGC- } \\
\quad 823\end{array}$ & $\begin{array}{l}300,600 \\
900 \mu \mathrm{g} / \mathrm{mL} \\
24 \mathrm{~h}\end{array}$ & $\uparrow \operatorname{ROS} \downarrow \Delta \Psi \mathrm{m}$ & {$[82]$} \\
\hline $\mathrm{G} 2 / \mathrm{M}$ & Flavonoids & Trifolirhizin & $\begin{array}{c}\text { (family: Fabaceae) } \\
\text { Sophora flavescens Aiton. Radix }\end{array}$ & MKN-45 & $\begin{array}{c}20,30,40 \\
\mu \mathrm{g} / \mathrm{mL} ; 48 \\
\mathrm{~h}\end{array}$ & $\begin{array}{l}\uparrow \text { caspase-9, -3, c- } \\
\text { PARP, p53, p38 } \\
\downarrow \text { EGFR, CDC2, cy- } \\
\text { clin } \mathrm{B}, \Delta \Psi \mathrm{m}\end{array}$ & {$[44]$} \\
\hline $\mathrm{G} 2 / \mathrm{M}$ & Phytosterols & Phloretin & & AGS & $\begin{array}{l}\text { 4. } 8,16 \mu \mathrm{M} \text {; } \\
24 \mathrm{~h}\end{array}$ & $\uparrow \operatorname{Bax} \downarrow$ Bcl-2 & {$[55]$} \\
\hline $\mathrm{G} 2 / \mathrm{M}$ & Phytosterols & $\begin{array}{l}\text { Tanshinone } \\
\text { IIA }\end{array}$ & $\begin{array}{c}\text { (family: Lamiaceae) Salviae miltiorrhiza } \\
\text { Bunge. Radix }\end{array}$ & AGS & $\begin{array}{l}2.0,3.7,5.5 \\
\mu \mathrm{g} / \mathrm{mL} ; 24 \\
\quad 48 \mathrm{~h}\end{array}$ & $\begin{array}{c}\uparrow T N F-\alpha, \text { Fas, p-p38, } \\
\text { p-JNK, p53, p21, } \\
\text { caspase- } 8,-3 \\
\downarrow \text { p-ERK, CDC2, cy- } \\
\text { clin A, cyclin B1 }\end{array}$ & {$[46]$} \\
\hline $\mathrm{G} 2 / \mathrm{M}$ & Terpenoids & $\begin{array}{c}\text { Deacetyli- } \\
\text { sovaltratum }\end{array}$ & $\begin{array}{c}\text { (family: Caprifoliaceae) } \\
\text { Patrinia heterophylla Bunge. }\end{array}$ & $\begin{array}{l}\text { (1) AGS } \\
\text { (2) HGC-27 }\end{array}$ & $\begin{array}{c}\text { (1) } 4,8,16 \\
\mu \mathrm{M} ; 24 \mathrm{~h} \\
(2) 10,20 \\
30 \mu \mathrm{M} ; 24 \\
\mathrm{~h}\end{array}$ & $\begin{array}{c}\uparrow \mathrm{p} 21 \text {, caspase-3, c- } \\
\text { PARP } \\
\downarrow \text { p-STAT3, pro- } \\
\text { caspase- } 9, \Delta \Psi \mathrm{m}\end{array}$ & {$[60]$} \\
\hline $\mathrm{G} 2 / \mathrm{M}$ & & $\begin{array}{l}\text { Ethanol and n- } \\
\text { hexane extract }\end{array}$ & $\begin{array}{l}\text { (family: Euphorbiaceae) Euphorbia lunu- } \\
\text { lata Bunge. }\end{array}$ & SGC-7901, ADR & $\begin{array}{c}10,20,40 \\
\mu \mathrm{g} / \mathrm{mL} ; 24 \\
\mathrm{~h}\end{array}$ & $\begin{array}{c}\uparrow \text { Bax, caspase- } 3,-8,- \\
9 \\
\downarrow \text { Bcl- } 2\end{array}$ & {$[76]$} \\
\hline
\end{tabular}

Bax, Bcl-2 like protein 4; Bcl-2, B-cell lymphoma 2; Fas, fasciclin; p-IkB $\alpha$, phospho-inhibitor of nuclear factor kappa B- $\alpha$; NF-kB, nuclear factor kappa-light-chain-enhancer of activated B cells; mTOR, mammalian target of rapamycin; Skp2, S-phase kinase-associated protein 2; CDKN2, cyclin-dependent kinase inhibitor 2; MEK1, mitogen activated protein kinase 1; MEKK3, mitogen activated protein kinase kinase 3; MEK5, mitogen activated protein kinase 5; Bid, BH3 interacting-domain death agonist; Bad, Bcl-2 associated agonist of cell; Bak, Bcl-2 antagonist/killer 1; cyt c, cytochrome c; c-caspase-3, cleaved caspase 3; c-caspase-8, cleaved caspase 8; c-caspase-9, cleaved caspase 9; AIF, apoptosis inducing factor; PARP, poly ADP ribose polymerase; Bcl-xL, B cell lymphoma-extra large ; IKB, inhibitor of nuclear factor kappa B- $\alpha$; c-PARP, cPARP, cleaved poly ADP ribose polymerase; Mcl-1, Myeloid cell leukemia-1; XIAP, X-linked inhibitor of apoptosis protein; RBBP4, retinoblastoma-binding protein 4; RUVBL1, RubA like AAA ATPase 1; NPM, Nucleophosmin 1; PI3K, Phosphoinositide 3-kinase; Akt1, protein kinase B 1; ROS, reactive oxygen species; $\Delta \Psi \mathrm{m}$, mitochondrial membrane potential; p-STAT3, phospho-Signal transducer and activator of transcription 3; TNF- $\alpha$, tumor necrosis factor-alpha; p-p38, phosphorylated p38; p-JNK, phosphorylated Jun N-terminal protein kinase; p-ERK, phosphorylated extracellular-signal-regulated kinase; EGFR, epidermal growth factor receptor; $\mathrm{CDC} 2$, cell division control protein 2. *The part of the plant used in this was not mentioned in the study. 


\section{Anti-angiogenesis effects of natural products in gastric cancer}

Angiogenesis is the most common pathway for new vessel formation in cancer [115]. Anti-angiogenic agents were studied and developed for anti-cancer therapies because angiogenesis can cause tumor growth [116]. Four in vitro and in vivo studies demonstrated anti-angiogenic property of natural products (Table 9, 10). Zhang et al. demonstrated the anti-angiogenesis property of arsenic trioxide with in vitro and in vivo models [117]. MGC803 and SGC-7901 cells treated with arsenic trioxide solution at doses of 2, 4, $8 \mu \mathrm{M}$ for 24 $\mathrm{h}$ showed increased level of forkhead box $\mathrm{O}$ 3a (FOXO3a) and decreased level of phosphorylated protein kinase B (p-Akt), VEGF, and MMP-9. This caused the reduction of gastric cancer cell viability, cell migration and angiogenesis. The anti-angiogenesis efficacy was confirmed in xenograft models inoculated with MGC-803 cells. After 14 days of treatment of $5 \mathrm{mg} / \mathrm{kg}$ arsenic trioxide solution every day daily, the experimental group had lighter and smaller tumors and showed lesser microvessel density than the control group rats. Xiaotan sanjie decoction is a Chinese medicine prescription originated from eleven herbs, Pinellia ternata Breitenbach Rhizoma (Araceae), Arisaema erubescens Schott Rhizoma (Araceae), Poria cocos (Peck) Ginns (Polyporaceae), Aurantii immaturus fructus (Rutaceae), Citri reticulatae viride pericardium (Rutaceae), Buthus martensii Karsch (Buthidae), Scolopendra subspinipes mutilans Linne. (Scolopendridae), Gallus gallus domesticus Brisson endothelium corneum (Phasianidae), Fritillariae cirrhosae D. Don bulbus (Liliaceae), Brassicae, Glycyrrhiza uralensis Fisch. (Febaceae) Yan et al. and Shi et al. reported that the Xiaotan sanjie decoction may inhibit angiogenesis by lowering expression of VEGF and VEGFR and down-regulating proteins interleukin 8 (IL-8), notch homolog 1 (Notch-1), hairy and enhancer of split-1 (Hes1), Ki-67, etc. in MKN-45 and HUVECs co-cultured with SGC-7901, respectively $[118,119]$. In addition, according to Yan et al., MKN-45 xenograft models treated with $4 \mathrm{~mL}$ of Xiaotan sanjie decoction for 2 weeks demonstrated reduction of tumor weight and angiogenesis in a dose-dependent manner. Huang et al. studied the anti-gastric cancer efficacy of cyperenoic acid extracted from the roots of Croton crassifolius Geiseler Radix (Euphorbiaceae) by treating the supercritical fluid extract and steam distillation extract of Croton crassifolius Geiseler Radix to zebrafish embryos [120]. Cyperenoic acid reduced vascular endothelial growth factor A (Vegfa or VEGF-A) genes by targeting the Vegfa-Kdr and Angpt-Tie signaling pathways. Notably, supercritical fluid extract showed stronger anti-angiogenic property than that of stream distillation extract, showing lower cytotoxicity toward both cancer and normal cells compared to the available drug, SU5416 (semaxanib). All studies suggest that inhibition of VEGF leads to anti-angiogenesis in various animal and cell line models. VEGFs take an important role in forming new blood vessels, including angiogenesis and vasculogenesis (Figure 3). These studies show some natural products can effectively downregulate certain VEGF subtypes including VEGFA156, VEGFA121, VEGFR1 and VEGFR2. These factors were modulated on mRNA expression levels. 


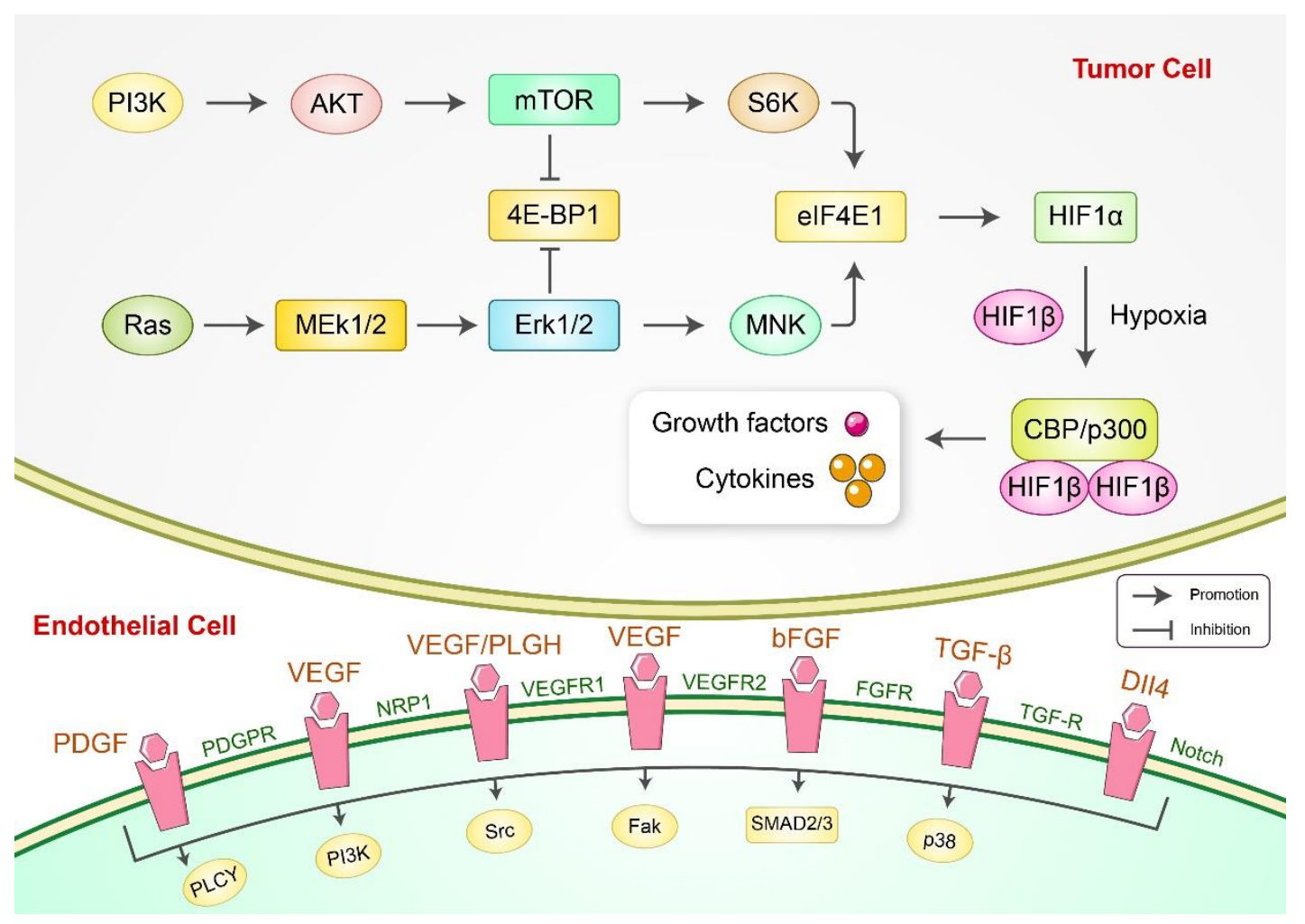

Figure 3. Schematic diagram of angiogenesis signaling pathways. PI3K, Phosphoinositide 3-kinase; Akt, protein kinase B; mTOR, mammalian target of rapamycin; S6K, S6 kinase; MEK1/2, mitogen activated protein kinase kinase 1/2; ERK1/2, extracellular signal-regulated kinase 1/2; MNK, Mitogen-activated protein kinase-interacting kinase; 4E-BP1, eIF4E-binding protein 1; elF4E1, Eukaryotic initiation factor 4E 1; HIF-1 $\alpha$, hypoxia-inducible factor-1 alpha; HIF-1 $\beta$, hypoxia-inducible factor-1 beta; CBP, CREB-binding protein; p300, CBP homolog; PDGF, platelet-derived growth factor; PDGFR, plateletderived growth factor receptor; VEGF, vascular endothelial growth factor; NRP1, neuropilin-1; PIGF, placental growth factor; VEGFR-1, vascular endothelial growth factor receptor-1; VEGFR-2, vascular endothelial growth factor receptor-2; bFGF, basic fibroblast growth factor; FGFR, fibroblast growth factor receptors; TGF- $\beta$, transforming growth factor beta; TGF-R, transforming growth factor receptor; Dll4, delta-like ligand 4. 
Table 9. Angiogenesis-Inhibiting Natural Products in vitro.

\begin{tabular}{|c|c|c|c|c|c|}
\hline Compound/ Extract & Source & Experimental Model & Dose; Duration & Mechanism & Reference \\
\hline Arsenic trioxide & & $\begin{array}{l}\text { (1) MGC-803 } \\
\text { (2) SGC-7901 }\end{array}$ & $2,4,8 \mu \mathrm{M} ; 24 \mathrm{~h}$ & $\begin{array}{c}\uparrow F O X O 3 \mathrm{a} \\
\downarrow \mathrm{p}-\mathrm{Akt}, \mathrm{VEGF}, \text { MMP-9 }\end{array}$ & [117] \\
\hline $\begin{array}{l}\text { Xiaotan sanjie } \\
\text { decoction }\end{array}$ & $\begin{array}{l}\text { (family: Araceae) Pinellia ternata } \text { Breitenbach Rhi- } \\
\text { zoma, (family: Araceae) Arisaema erubescens } \text { Schott } \\
\text { Rhizoma, (family: Polyporaceae) Poria cocos (Peck) } \\
\text { Ginns, (family: Rutaceae) Aurantii immaturus fructus, } \\
\text { (family: Rutaceae) Citri reticulatae viride pericardium, } \\
\text { (family: Buthidae) Buthus martensii Karsch, (Scolopen- } \\
\text { dridae) Scolopendra subspinipes mutilans Linne., (fam- } \\
\text { ily: Phasianidae) Gallus gallus domesticus Brisson en- } \\
\text { dothelium corneum, (family: Liliaceae) Fritillariae cir- } \\
\text { rhosae D.Don bulbus, Brassicae, (family: Febaceae) } \\
\text { Glycyrrhiza uralensis Fisch. }\end{array}$ & MKN-45 & $\begin{array}{c}1.46,2.92,5.84 \mathrm{~g} / \mathrm{mL} \\
24,48,72 \mathrm{~h}\end{array}$ & $\begin{array}{l}\downarrow \text { IL-8, Notch-1, Hes1, VEGF, VEGF- } \\
\text { A, VEGFR-1, VEGFR-2, Ki-67 }\end{array}$ & [118] \\
\hline $\begin{array}{l}\text { Xiaotan sanjie } \\
\text { decoction }\end{array}$ & $\begin{array}{l}\text { (family: Araceae) Pinellia ternata Breitenbach Rhi- } \\
\text { zoma, (family: Araceae) Arisaema erubescens Schott } \\
\text { Rhizoma, (family: Polyporaceae) Poria cocos (Peck) } \\
\text { Ginns, (family: Rutaceae) Aurantii immaturus fructus, } \\
\text { (family: Rutaceae) Citri reticulatae viride pericardium, } \\
\text { (family: Buthidae) Buthus martensii Karsch, (Scolopen- } \\
\text { dridae) Scolopendra subspinipes mutilans Linne., (fam- } \\
\text { ily: Phasianidae) Gallus gallus domesticus Brisson en- } \\
\text { dothelium corneum, (family: Liliaceae) Fritillariae cir- } \\
\text { rhosae D.Don bulbus, Brassicae, (family: Febaceae) } \\
\text { Glycyrrhiza uralensis Fisch. }\end{array}$ & $\begin{array}{l}\text { HUVECs } \\
\text { co-cultured } \\
\text { with SGC-7901 }\end{array}$ & $10 \%$ serum; $24 \mathrm{~h}$ & $\begin{array}{l}\downarrow \text { IL-8, NOTCH-1, VEGF-A, VEGFR- } \\
1 \text {, VEGFR-2 }\end{array}$ & [119] \\
\hline
\end{tabular}

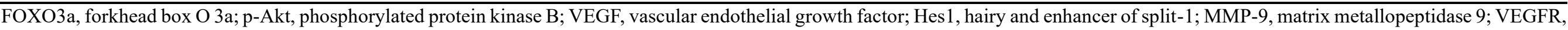
vascular endothelial growth factor receptor; IL-8, interleukin-8; SFE, supercritical fluid extract; SDE, steam distillation extract 
Table 10. Angiogenesis-Inhibiting Natural Products in vivo

\begin{tabular}{|c|c|c|c|c|c|c|}
\hline $\begin{array}{l}\text { Classifica- } \\
\text { tion }\end{array}$ & $\begin{array}{l}\text { Compound/ Ex- } \\
\text { tract }\end{array}$ & Source & Experimental Model & Dose; Duration & Mechanism & Reference \\
\hline Terpenoid & $\begin{array}{l}\text { Cyperenoic acid / } \\
\text { SFE or SDE }\end{array}$ & $\begin{array}{c}\text { (family: Euphorbiaceae) } \\
\text { Croton crassifolius Geiseler Radix }\end{array}$ & Zebrafish embryos & $\begin{array}{c}3.75,7.5,15 \\
\mu \mathrm{g} / \mathrm{mL} ; 24,48 \mathrm{~h}\end{array}$ & $\begin{array}{l}\downarrow \text { Vegfa156, Vegfa121, Kdr, } \\
\text { Angpt1, Angpt2, Tie1, Tie2 }\end{array}$ & {$[120]$} \\
\hline & Arsenic trioxide & & $\begin{array}{l}\text { BALB/C-nu/nu nude } \\
\text { mice / MGC-803 }\end{array}$ & $\begin{array}{c}5 \mathrm{mg} / \mathrm{kg} / \mathrm{day} ; 14 \\
\text { days }\end{array}$ & $\uparrow \mathrm{FOXO3a}$ & [117] \\
\hline & $\begin{array}{l}\text { Xiaotan sanjie de- } \\
\text { coction }\end{array}$ & $\begin{array}{l}\text { (family: Araceae) Pinellia ternata } \\
\text { Breitenbach Rhizoma, (family: Araceae) } \\
\text { Arisaema erubescens Schott Rhizoma, } \\
\text { (family: Polyporaceae) Poria cocos } \\
\text { (Peck) Ginns, (family: Rutaceae) Au- } \\
\text { rantii immaturus fructus, (family: Ru- } \\
\text { taceae) Citri reticulatae viride pericar- } \\
\text { dium, (family: Buthidae) Buthus mar- } \\
\text { tensii Karsch, (Scolopendridae) Scolo- } \\
\text { pendra subspinipes mutilans Linne., } \\
\text { (family: Phasianidae) Gallus gallus do- } \\
\text { mesticus Brisson endothelium corneum, } \\
\text { (family: Liliaceae) Fritillariae cirrhosae } \\
\text { D.Don bulbus, Brassicae, (family: Fe- } \\
\text { baceae) Glycyrrhiza uralensis Fisch. }\end{array}$ & $\begin{array}{l}\text { SD rats, nude mice / } \\
\text { MKN-45 }\end{array}$ & $\begin{array}{c}1.46,2.92 \text { and } \\
5.84 \mathrm{~g} / \mathrm{mL}, 4 \mathrm{~mL} \\
\text { daily; } 8 \text { weeks }\end{array}$ & $\downarrow$ Notch-1, Hes1 & [118] \\
\hline
\end{tabular}




\section{Anti-metastasis effects of natural products in gastric cancer}

Metastasis is a major contributor of death in cancer patients, arising from a growing tumor from which cells escape to distant organs of body [121]. Targeting metastasis is an attractive strategy in cancer treatment. Sixteen studies highlighted the anti-metastatic ability of diverse natural products in vitro and in vivo models. Epithelial-mesenchymal transition (EMT) was inhibited in many cases while cell cycle arrest and other blockages of tumor proliferations were also observed (Table 11,12). Evodiamine is a chemical compound extracted from Evoida rutaecarpa (Rutaceae) [122]. The compound suppressed epithelialmesenchymal transition of AGS and SGC-7901 gastric cancer cells via inhibition of $W n t / \beta$-catenin signaling pathway (Figure 4 ). Sulforaphane is an organosulfur compound extracted from Brassica oleracea var. italica Plenk (Brassicaceae) [123]. The compound exerted anti-metastatic ability on AGS and MKN-45 cells when treated at dose of 31.25, 62.5, $125,250 \mu \mathrm{g} / \mathrm{mL}$ for $48 \mathrm{~h}$. It upregulated caudal type homobox 1 (CDX1), caudal type homobox 2 (CDX2), miR-326, and miR-9. Isoliquiritigenin, a phenol found in Glycyrrhiza glabra (Fabaceae), inhibited tumor migration and metastasis on MKN-28 in vitro when treated at dose of $20 \mu \mathrm{M}$ for $24,48,72 \mathrm{~h}$ [124]. The compound suppressed the phosphoinositide-3 kinase (PI3K)/AKT/mTOR signaling pathway. Dehydroeffusol is a benzenoid derived from Juncus effusus L. Radix et Medulla (Juncaceae) [125]. The compound was tested at the dose of 12, 24, $48 \mu \mathrm{M}$ for 24 on AGS and SGC-7901 cells in vitro, which resulted in inhibition of matrix metalloproteinase 2 (MMP-2) and reduction of cell-to-cell adherent junction. Downregulation of VE-cadherin and MMP-2 expression was observed. Paenol, the water extract of Paeonia suffruticosa Andr. (Paeoniaceae) and Cynanchum paniculatum K. Schum (Asclepiadaceae), was treated on BGC-823 cells at dose of $0.1,0.2,0.4 \mathrm{mg} / \mathrm{mL}$ for $24,48 \mathrm{~h}$ [64]. The extract downregulated MMP-2 and MMP-9, leading to inhibition of cell proliferation and migration. Baicalein is a well-known flavone found in the roots of Scutellaria baicalensis Georgi Radix (Lamiaceae) [126]. A 2014 study by Chen F. et al. showed the compound restrains motility, migration, and invasion of AGS gastric cancer cells via downregulation of N-cadherin, vimentin, ZEB1, ZEB2 and TGF- $\beta /$ Smad4. Baicalein was treated in vitro at dose of $25,50 \mu \mathrm{M}$ for $24 \mathrm{~h}$. Andrographolide is a labdane diterpenoid from herb Andrographis paniculata Nees Herba (Acanthaceae) [127]. Dai L et al. demonstrated that the compound inhibits proliferation and metastasis of gastric cancer SGC-7901 via cell cycle arrest, when treated in vitro at dose of $5,20,40 \mu \mathrm{g} / \mathrm{mL}$ for $24,48,72 \mathrm{~h}$. Upregulation of Bax, Bik, TIMP-1/2 and downregulation of Bcl-2, CD147, MMP-2, MMP-9, survivin were observed. Wang $\mathrm{G}$ et al. demonstrated that a triterpenoid extracted from Melia toosendan Sieb et Zucc (Meiliaceae) named toosendanin has anti-metastatic capability on SGC-7901 cells in vitro [67]. The compound inhibited epithelial-mesenchymal transition of gastric cancer by upregulating miR-200a, E-cadherin and suppressing $\beta$-catenin when treated at dose of $0.5,1 \mu \mathrm{M}$ for $48 \mathrm{~h}$. Tangerines, grapefruits, lemons and oranges contain low-molecular-weight citrus pectin (LCP) [128]. Wang et al. demonstrated that anti-metastatic effect of LCP by treating it on AGS cells in vitro at dose of $0.625,1.25,2.5,5,10 \mathrm{mg} / \mathrm{mL}$ for $24 \mathrm{~h}$. Epithelial-mesenchymal transition was inhibited followed by downregulation of $\mathrm{Cy}-$ clin B1, galectin-3 (GAL-3) and Bcl-xL. LCP had both apoptotic and anti-metastatic effects. N-butylidenephthalide is a compound extracted from the roots of Angelica sinensis (Oliv.) Diels Radix (Apiaceae) [63]. When treated at dose of $50 \mu \mathrm{g} / \mathrm{mL}$ for $24,48 \mathrm{~h}$ on AGS, $75 \mu \mathrm{g} / \mathrm{mL}$ for $24,48 \mathrm{~h}$ on NCI-N87 and 25, 50, $75 \mu \mathrm{g} / \mathrm{mL}$ for $48 \mathrm{~h}$ on TSGH-9201, inhibition of tumor metastasis was observed. The compound promoted E-cadherin expression while downregulating $\mathrm{N}$-cadherin and vimentin slug. It is unclear whether natural products exert anti-metastatic effect in a multi-target manner. Further study is required to distinguish the specific mechanism. The primary mechanism observed was through the inhibition of cadherin-catenin adhesion. Compounds and extracts including baicalein, Celastrus orbiculatus extract, dehydroeffusol, etc., down-regulated $\mathrm{N}$-cadherin, VE-cadherin, $\beta$-catenin and other related factors $[125,126,129,130]$. Activity of E-cadherin was repressed on the other hand, which inhibits EGFR kinase activity. The mechanism leads to downstream regulation of multiple growth factor related activities, which is associated with anti-metastatic activities of such natural products. Notably, Shi J et al. demonstrated the Xiaotan 
Sanjie decoction down-regulated VEGF-A, VEGFR-1 and VEGFR-2 [119]. In other aspect, the Bcl-2 family proteins were also found to play role in anti-metastatic effects of natural products. For example, products such as andrographolide and Xian Tan He Wei elevated Bax protein activity while inhibiting Bcl-2 protein [127, 131]. Many other factors including PI3K, Akt, Rac1, CDX1/2 play a role in anti-metastatic activity of natural products, some of which are also related to apoptosis of tumor cells. For example, Lyu et al. reported the paeonol extract suppressed the expression of MMP-2 and MMP-9, which lead to inhibition of proliferation and so inhibition of metastasis (Figure 4) [64]. It is unclear whether natural products exert anti-metastatic effect in a multi-target manner. Further study is required to distinguish the specific mechanism.

Two studies by Zhu Y et al. demonstrated ethyl acetate extract of Celastrus orbiculatus Thunb. Rhizoma (Celastraceae) inhibits TGF- $\beta 1$ induced epithelial-mesenchymal transition of gastric cancer SGC-7901 [129, 130]. In both studies, the cells were treated with 5, 10, 20 $\mu \mathrm{g} / \mathrm{mL}$ dose of the extract for $24 \mathrm{~h}$. Promotion of E-cadherin and downregulation of $\mathrm{N}$ cadherin, vimentin, NF- $\mathrm{KB}$ and HSP27 were observed. Triphala is a decoction derived from Terminalia chebula Retz. Fructus (Combretaceae), Terminalia bellerica (Gaertn.) Roxb Fructus (Combretaceae) and Phyllanthus emblica Linn. Fructus (Phyllanthaceae) [132]. The decoction downregulated EGFR, Akt and ERK thus inhibiting tumor proliferation and suppressing cell migration both in vivo and in vitro. Triphala was treated on MGC-803 at dose of $50,100,150 \mu \mathrm{g} / \mathrm{mL}$ for $48 \mathrm{~h}$, and tested on Danio rerio at dose of 50, 100, $150 \mu \mathrm{g} / \mathrm{mL}$. Xiaotan Sanjie is a traditional Chinese decoction composing Pinelliae rhizome (Araceae), Arisaema erubescens Schott Rhizoma (Araceae), Poria cocos (Peck) Ginns (Polyporaceae), Aurantii immaturus Fructus (Rutaceae), Citri reticulatae viride pericarpium (Rutaceae), Scolopendra subspinipes mutilans Linne. (Scolopendridae), Scolopendra (Scolopendridae), Gallus gallus demusticus Brisson Endothelium corneum (Phasianidae), Fritillariae cirrhosae Bulbus (Liliaceae), Semen brassicae and Glycyrrhiza uralensis Fisch (Febaceae) [119]. 10\% xiaotan sanjie decoction serum was treated to SGC-7901 gastric cancer cells for 24 hours. It inhibited cell adhesion, migration, and invasion by downregulating IL-8, NOTCH-1, VEGF-A, VEGFR-1 and VEGFR-2. Xu et al. demonstrated the traditional Chinese decoction Xiao Tan He Wei also possesses anti-metastatic effect on tumor-induced animals [90]. Inhibition of invasion and metastasis was detected when wistar rats bearing precancerous lesions of gastric carcinoma were given the decoction orally. Jianpi Bushen is a traditional Chinese decoction made from extracts of species including Codonopsis pilosula Nannfeldt Radix (Campanulaceae), Lycium chinense Miller Fructus (Solanaceae), Atractylodis Macrocephalae Koidzumi Rhizoma (Solanaceae), Ligustri lucidum Aiton Fructus (Oleaceae), Cuscuta chinensis Lamark Semen (Convolvulaceae), Psoralea corylifolia Linn. Semen (Leguminosae/Fabacea) [133]. The treatment, tested on strain- 615 model in vivo at dose of $20 \mathrm{~g} / \mathrm{kg}$ for 7 days, inhibited cancer cell motility and inhibited metastasis by downregulating Ras-related botulinum toxin substrate 1 (Rac1), cell division control protein 42 homolog (Cdc42), stromal cell-derived factor 1 (SDF-1) and fibronectin (FN). Apoptosis and cell cycle arrest was also observed as described above. 


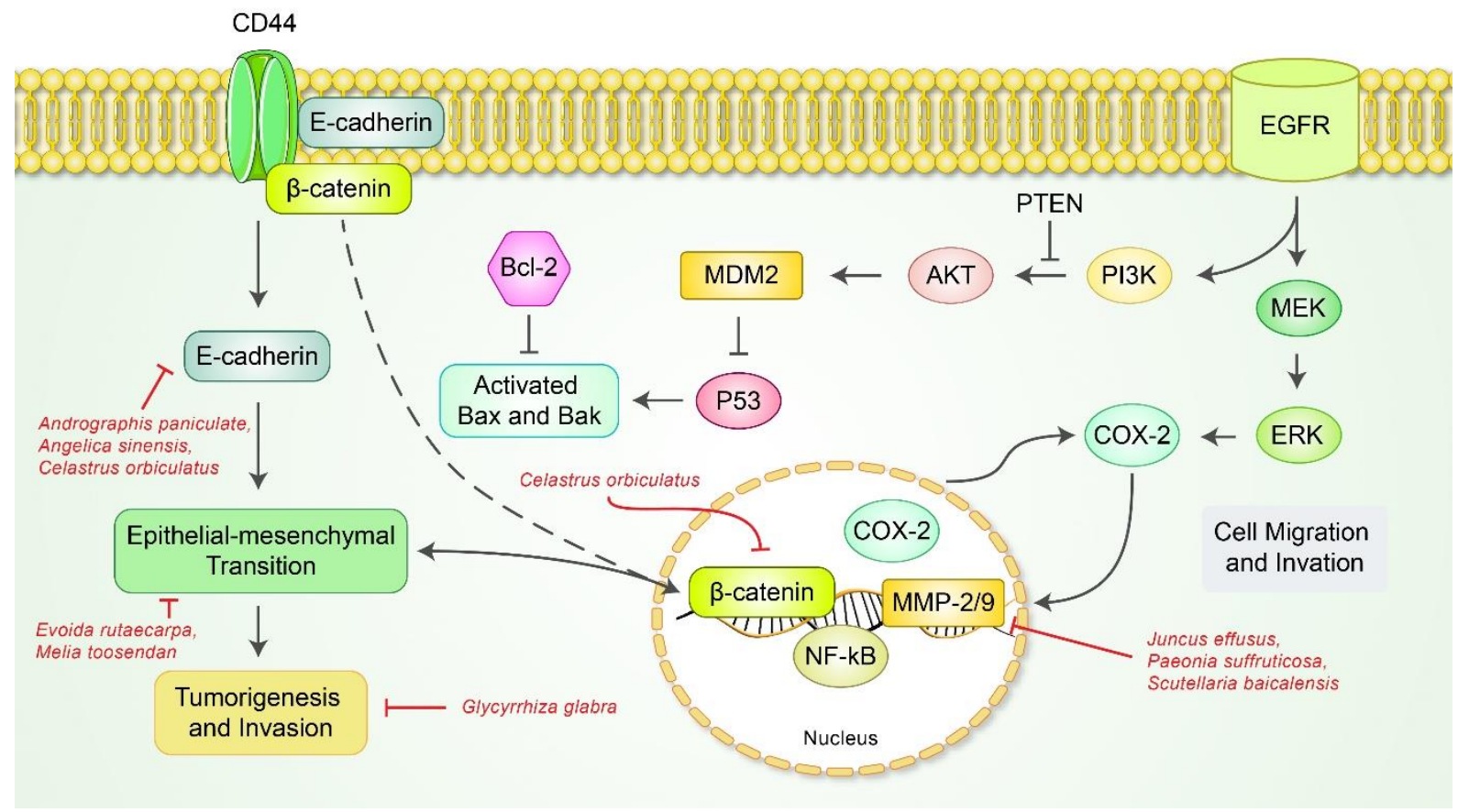

Figure 4. Schematic diagram of metastasis signaling pathways. Akt, protein kinase B; Bak, Bcl-2 antagonist/killer 1; Bax, Bcl-2-like protein 4; Bcl-2, B-cell lymphoma 2; CD44, homing cell adhesion molecule; COX-2, cyclooxygenase 2; EGFR, epidermal growth factor receptor; ERK, extracellular signal-regulated kinase; MDM2, murine double minute 2; MEK, miTable 2. matrix metalloproteinase-2/9; NF- $\kappa \mathrm{B}$, nuclear factor kappa-B; PI3K, phosphoinositide 3-kinase; PTEN, phosphatase and tensin homolog. 
Table 11. Metastasis-inhibiting natural products in vitro.

\begin{tabular}{|c|c|c|c|c|c|c|c|}
\hline Classification & $\begin{array}{l}\text { Compound/ } \\
\text { Extract }\end{array}$ & Source & $\begin{array}{l}\text { Experimental } \\
\text { Model }\end{array}$ & $\begin{array}{l}\text { Dose; Dura- } \\
\quad \text { tion }\end{array}$ & Efficacy & Mechanism & Reference \\
\hline Alkaloids & Evodiamine & $\begin{array}{c}\text { (family: Rutaceae) Tetradium ruti- } \\
\text { carpum }\end{array}$ & $\begin{array}{l}\text { AGS, SGC- } \\
7901\end{array}$ & $2 \mu \mathrm{M} ; 48 \mathrm{~h}$ & Inhibition of EMT & $\begin{array}{l}\downarrow \beta \text {-catenin, cyclin } \\
\text { D1, c-Myc }\end{array}$ & [122] \\
\hline $\begin{array}{l}\text { Organosulfur } \\
\text { compounds }\end{array}$ & Sulforaphane & $\begin{array}{l}\text { (family: Brassicaceae) Brassica } \\
\text { oleracea } \text { var. italica Plenk }\end{array}$ & AGS, MKN-45 & $\begin{array}{c}31.25,62.5 \\
125,250 \\
\mu \mathrm{g} / \mathrm{mL} ; 48 \mathrm{~h}\end{array}$ & Inhibition of metastasis & $\begin{array}{c}\uparrow \mathrm{CDX} 1, \mathrm{CDX} 2 \\
\uparrow \mathrm{miR}-326, \text { miR-9 }\end{array}$ & [123] \\
\hline Polyphenols & Isoliquiritigenin & $\begin{array}{c}\text { (family: Fabaceae) Glycyrrhiza glabra } \\
\text { Radix }\end{array}$ & MKN-28 & $\begin{array}{l}20 \mu \mathrm{M} ; 24 \\
48,72 \mathrm{~h}\end{array}$ & $\begin{array}{c}\text { Inhibition of migration, invasion } \\
\text { Induction of apoptosis and au- } \\
\text { tophagy }\end{array}$ & $\begin{array}{l}\downarrow \text { Caspase-3, Bax, } \\
\text { Bcl-2, PI3K, Akt, } \\
\text { mTOR }\end{array}$ & [124] \\
\hline Polyphenols & Dehydroeffusol & $\begin{array}{l}\text { (family: Juncaceae) Juncus effusus L. } \\
\text { Radix et Medulla }\end{array}$ & $\begin{array}{l}\text { AGS, SGC- } \\
7901\end{array}$ & $\begin{array}{l}12,24,48 \\
\mu \mathrm{M} ; 24 \mathrm{~h}\end{array}$ & $\begin{array}{l}\text { Reduction of cell-cell adherent } \\
\text { junction }\end{array}$ & $\begin{array}{l}\downarrow \text { VE-cadherin, } \\
\text { MMP-2 }\end{array}$ & [125] \\
\hline Polyphenols & Paeonol / extract & $\begin{array}{l}\text { (family: Paeoniaceae) Paeonia suffruti- } \\
\text { cosa Andr. Cortex, } \\
\text { (family: Asclepiadaceae) Cynanchum } \\
\text { paniculatum K. Schum Radix }\end{array}$ & BGC-823 & $\begin{array}{l}0.1,0.2,0.4 \\
\mathrm{mg} / \mathrm{mL} ; 24 \\
\quad 48 \mathrm{~h}\end{array}$ & $\begin{array}{l}\text { Inhibition of proliferation, inva- } \\
\text { sion, and migration } \\
\text { Induction of apoptosis }\end{array}$ & $\downarrow$ MMP-2, MMP-9 & [64] \\
\hline Polyphenols & Baicalein & $\begin{array}{c}\text { (Lamiaceae) Scutellaria baicalensis } \\
\text { Georgi Radix }\end{array}$ & AGS & $\begin{array}{l}25,50 \mu \mathrm{M} \\
24 \mathrm{~h}\end{array}$ & $\begin{array}{c}\text { Inhibition of motility, migration, } \\
\text { invasion }\end{array}$ & $\begin{array}{c}\downarrow N \text {-cadherin, vi- } \\
\text { mentin, ZEB1, } \\
\text { ZEB2, TGF- } \\
\beta / \text { Smad4 }\end{array}$ & [126] \\
\hline Terpenoids & Andrographolide & $\begin{array}{l}\text { (family: Acanthaceae) Andrographis } \\
\text { paniculata Nees Herba }\end{array}$ & SGC-7901 & $\begin{array}{c}5,20,40 \\
\mu \mathrm{g} / \mathrm{mL} ; 24 \\
48,72 \mathrm{~h}\end{array}$ & $\begin{array}{l}\text { Inhibition of proliferation, inva- } \\
\text { sion, metastasis }\end{array}$ & $\begin{array}{c}\text { Bax, Bik, TIMP- } \\
1 / 2 \\
\downarrow \text { Bcl-2, CD147, } \\
\text { MMP-2, MMP-9, } \\
\text { survivin }\end{array}$ & [127] \\
\hline
\end{tabular}




\begin{tabular}{|c|c|c|c|c|c|c|c|}
\hline Terpenoids & Toosendanin & $\begin{array}{l}\text { (family: Meliaceae) Melia toosendan } \\
\text { Sieb et Zucc Cortex et Fructus }\end{array}$ & SGC-7901 & $\begin{array}{l}0.5,1 \mu \mathrm{M} \\
\quad 48 \mathrm{~h}\end{array}$ & $\begin{array}{c}\text { Inhibition of invasion, migra- } \\
\text { tion, EMT } \\
\text { Induction of apoptosis and cell } \\
\text { cycle arrest }\end{array}$ & $\begin{array}{c}\begin{array}{c}\uparrow \text { E-cadherin } \downarrow \beta \text { - } \\
\text { catenin }\end{array} \\
\uparrow \mathrm{miR}-200 \mathrm{a}\end{array}$ & [67] \\
\hline Terpenoids & $\begin{array}{l}\text { Low-molecular- } \\
\text { weight citrus pec- } \\
\text { tin (LCP) }\end{array}$ & Citri Pericarpium* & AGS & $\begin{array}{c}0.625,1.25 \\
2.5,5,10 \\
\mathrm{mg} / \mathrm{mL} ; 24 \mathrm{~h}\end{array}$ & Inhibition of EMT & $\begin{array}{l}\downarrow \text { Cyclin B1, GAL- } \\
\text { 3, Bcl-xL }\end{array}$ & {$[128]$} \\
\hline \multirow[t]{3}{*}{ Terpenoids } & $\begin{array}{l}\text { N-Butylideneph- } \\
\text { thalide }\end{array}$ & $\begin{array}{c}\text { (family: Apiaceae) Angelica sinensis } \\
\text { (Oliv.) Diels Radix }\end{array}$ & $\begin{array}{c}\text { (1) AGS } \\
\text { (2) NCI-N87 } \\
\text { (3) TSGH-9201 }\end{array}$ & $\begin{array}{c}\text { (1) } 50 \\
\mu \mathrm{g} / \mathrm{mL} ; 24, \\
48 \mathrm{~h} \\
\text { (2) } 75 \\
\mu \mathrm{g} / \mathrm{mL} ; 24, \\
48 \mathrm{~h} \\
\text { (3) } 0,25,50, \\
75 \mu \mathrm{g} / \mathrm{mL} ; \\
48 \mathrm{~h}\end{array}$ & $\begin{array}{c}\text { Inhibition of migration, invasion } \\
\text { and EMT }\end{array}$ & $\begin{array}{l}\uparrow \text { E-cadherin } \\
\downarrow N \text {-cadherin, vi- } \\
\text { mentin slug }\end{array}$ & {$[63]$} \\
\hline & $\begin{array}{l}\text { Ethyl acetate ex- } \\
\text { tract }\end{array}$ & $\begin{array}{c}\text { (family: Celastraceae) Celastrus orbic- } \\
\text { ulatus Thunb. Rhizoma }\end{array}$ & SGC-7901 & $\begin{array}{l}5,10,20 \\
\mu \mathrm{g} / \mathrm{mL} ; 24 \mathrm{~h}\end{array}$ & Inhibition of EMT & $\begin{array}{l}\uparrow \text { E-cadherin } \\
\downarrow N \text {-cadherin, vi- } \\
\text { mentin, NF- } \kappa B\end{array}$ & {$[129,130]$} \\
\hline & Triphala decoction & $\begin{array}{c}\text { (family: Combretaceae) Terminalia } \\
\text { chebula Retz. Fructus, (family: Com- } \\
\text { bretaceae) Terminalia bellerica } \\
\text { (Gaertn.) Roxb. Fructus, (family: Phyl- } \\
\text { lanthaceae) Phyllanthus emblica Linn. } \\
\text { Fructus }\end{array}$ & MGC-803 & $\begin{array}{l}50,100,150 \\
\mu \mathrm{g} / \mathrm{mL} ; 48 \mathrm{~h}\end{array}$ & Inhibition of migration & $\downarrow$ EGFR, Akt, ERK & [132] \\
\hline
\end{tabular}


(family: Araceae) Pinellia ternata

Breitenbach Rhizoma, (family: Araceae) Arisaema erubescens Schott Rhizoma, (family: Polyporaceae) Poria cocos (Peck) Ginns, (family: Rutaceae) Aurantii immaturus fructus, (family:

Xiaotan Sanjie Rutaceae) Citri reticulatae viride peri-

decoction cardium, (family: Buthidae) Buthus martensii Karsch, (Scolopendridae) Scolopendra subspinipes mutilans Linne., (family: Phasianidae) Gallus gallus domesticus Brisson endothelium corneum, (family: Liliaceae) Fritillariae cirrhosae D.Don bulbus, Brassicae, (family: Febaceae) Glycyrrhiza ura-

lensis Fisch.
HUVECs co-

cultured with

SGC-7901
$10 \%$ serum;

$24 \mathrm{~h}$
Inhibition of adhesion, migration and invasion $\downarrow$ IL-8, NOTCH-1, VEGF-A,

VEGFR-1,

VEGFR-2

(family: Umbelliferae) Bupleurum falcatum Linne. Radix, (family: Araceae) Pinellia ternata (Thunb.) Makino Rhizoma, (family: Polyporaceae) Poria cocos Wolf., (family: Ranunculaceae)

Xiao Tan He Wei Coptis chinensis Franch., (family: Rubiaceae) Oldenlandia diffusa (Willd.) Decoction Roxb. Herba, (family: Compositae) Taraxaci Herba*, (family: Lauraceae) Cinnamonun cassia J. Presl twig, (family: Polygonaceae) Rhubarb*, (family: Paeoniaceae) Paeonia lactiflora Pallas., (family: Leguminosae/Fabaceae)

$0.01,0.02$, $0.04,0.08$,

GES-1
$0.16,0.32$,

$2.56 \mathrm{~g} / \mathrm{L} ; 24$

$48,72,96 \mathrm{~h}$ $\uparrow$ Bax, c-caspase-3,

$\mathrm{IkB}$

$\downarrow \mathrm{Bcl}-2, \mathrm{p} 65, \mathrm{NF}-$

$\kappa \mathrm{B}$

Glycyrrhizae uralensis Fischer.

B-catenin, catenin beta-1; CDX1, caudal type homeobox 1; CDX2, caudal type homeobox 2; miR-326, microRNA 326; miR-9, microRNA 9; Bax, bcl-2-like protein 4; Bcl-2, B-cell lymphoma 2; PI3K, phosphoinositide 3-kinase; Akt, protein kinase B; mTOR, mammalian target of rapamycin; VE-cadherin, vascular endothelial-cadherin; MMP-2, matrix metalloproteinase-2; MMP-9, matrix metalloproteinase-9; N-cadherin, neural cadherin; ZEB1, zinc finger E-box binding homeobox 1; ZEB2, zinc finger E-box binding homeobox 2; TGF- $\beta$ /Smad4, transforming growth factor beta 1/SMAD family member 4; Bik, Bcl-2 interacting killer; EMT, epithelial-mesenchymal transition; TIMP-1/2, tissue inhibitors of matrix metalloproteinase-1/2; CD147, cluster of differentiation 147; E-cadherin, epithelia cadherin; miR-200a, microRNA 200a; GAL-3, galectin-3; Bcl-xL, B-cell lymphoma-extra large; NF- $\mathrm{kB}$, nuclear factor $\kappa$-light-chain-enhancer of activated B cells; EGFR, epidermal growth factor receptor; ERK, extracellular signal-regulated kinase; IL-8, interleukin-8; NOTCH-1, Notch homolog 1; VEGF-A, vascular endothelial growth 
factor-A; VEGFR-1, vascular endothelial growth factor receptor-1; VEGFR-2, vascular endothelial growth factor receptor-2; c-caspase-3, cleaved caspase-3; IкB, Inhibitor of NF-кB; p65, nuclear factor NF-кB p65 subunit; Rac1, Ras-related botulinum toxin substrate $1 .{ }^{*}$ Citri pericarpium is derived from several citrus fruits. The exact species used in the research was not identified.

Table 12. Metastasis-inhibiting natural products in vivo.

\begin{tabular}{|c|c|c|c|c|c|c|}
\hline $\begin{array}{c}\text { Compound/ } \\
\text { Extract }\end{array}$ & Source & $\begin{array}{c}\text { Experimental } \\
\text { Model }\end{array}$ & $\begin{array}{c}\text { Dose; Dura- } \\
\text { tion }\end{array}$ & Efficacy & Mechanism & Reference \\
\hline $\begin{array}{l}\text { Triphala de- } \\
\text { coction }\end{array}$ & $\begin{array}{l}\text { (family: Combretaceae) Terminalia chebula } \text { Retz. Fructus, } \\
\text { (family: Combretaceae) Terminalia bellerica (Gaertn.) } \\
\text { Roxb. Fructus, (Phyllanthaceae) Phyllanthus emblica Linn. } \\
\text { Fructus }\end{array}$ & $\begin{array}{l}\text { Zebrafish } \\
\text { (Danio rerio) / } \\
\text { MGC-803 }\end{array}$ & $\begin{array}{l}50,100 \\
150 \mu \mathrm{g} / \mathrm{mL} \\
48 \mathrm{~h}\end{array}$ & $\begin{array}{l}\text { Inhibition } \\
\text { of migra- } \\
\text { tion }\end{array}$ & $\begin{array}{c}\downarrow \text { EGFR, Akt, } \\
\text { ERK }\end{array}$ & [132] \\
\hline $\begin{array}{l}\text { Xiao Tan He } \\
\text { Wei } \\
\text { decoction }\end{array}$ & $\begin{array}{l}\text { (family: Umbelliferae) Bupleurum falcatum Linne. Radix, } \\
\text { (family: Araceae) Pinellia ternata (Thunb.) Makino Rhi- } \\
\text { zoma, (family: Polyporaceae) Poria cocos Wolf., (family: } \\
\text { Ranunculaceae) Coptis chinensis Franch., (family: Rubia- } \\
\text { ceae) Oldenlandia diffusa (Willd.) Roxb. Herba, (family: } \\
\text { Compositae) Taraxaci Herba*, (family: Lauraceae) Cinna- } \\
\text { monun cassia J. Presl twig, (family: Polygonaceae) Rhu- } \\
\text { barb*, (family: Paeoniaceae) Paeonia lactiflora Pallas., } \\
\text { (family: Leguminosae/Fabaceae) Glycyrrhizae uralensis } \\
\text { Fischer. }\end{array}$ & Wistar rats & $\begin{array}{c}3.4 \mathrm{~mL} / \mathrm{kg} ; 2 \\
\text { weeks }\end{array}$ & $\begin{array}{l}\text { Induction } \\
\text { of cell cy- } \\
\text { cle arrest }\end{array}$ & $\begin{array}{l}\uparrow \text { Bax, c-caspase- } \\
\text { 3, IкB } \\
\downarrow \text { Bcl-2, p65, NF- } \\
\kappa \mathrm{B}\end{array}$ & [90] \\
\hline $\begin{array}{l}\text { Jianpi Bushen } \\
\text { decoction }\end{array}$ & $\begin{array}{c}\text { (family: Campanulaceae) Codonopsis pilosula Nannfeldt } \\
\text { Radix, (family:Solanaceae) Lycium chinense Miller Fructus, } \\
\text { (family: compositae) Atractylodis macrocephalae Koidzumi } \\
\text { Rhizoma, (family: Oleaceae) Ligustri lucidum Aiton Fructus, } \\
\text { (family: Convolvulaceae) Cuscuta chinensis Lamark Semen, } \\
\text { (family: Leguminosae/Fabaceae) Psoralea corylifolia Linn. } \\
\text { Semen }\end{array}$ & $\begin{array}{c}\text { Strain-615 / } \\
\text { MFC }\end{array}$ & $\begin{array}{c}20 \mathrm{~g} / \mathrm{kg} ; 7 \\
\text { days }\end{array}$ & $\begin{array}{l}\text { Inhibition } \\
\text { of motility }\end{array}$ & $\begin{array}{c}\downarrow \mathrm{Rac} 1, \mathrm{Cdc} 42, \\
\text { SDF-1, FN }\end{array}$ & [133] \\
\hline
\end{tabular}

control protein 42 homolog; SDF-1, stromal cell-derived factor 1; FN, fibronectin. 


\section{Chemotherapy resistance and natural products in gastric cancer}

Drug resistance has been an important issue in cancer treatment. It is known as a primary cause limiting cancer treatment [134]. Several studies have indicated that natural products could be used along with the primary drug to overcome drug resistance and reinforce its efficacy. Previous studies focused on how natural products can support conventional antibiotics by suppressing resistance [135]. This review highlighted natural products' potential in handling cancer chemotherapy resistance (Table 13, 14). Isorhamnetin, a flavonoid metabolite of quercetin commonly found in onions, minimized the apoptotic effects of capecitabine via inhibition of NF- $\kappa B$ and various NF- $\kappa B$ regulated gene products in tumor cells [136]. Attenuation of capecitabine resistance was also observed in an animal model in which athymic nu/nu female mice grafted with SNU-5 were treated with isorhamnetin $(1 \mathrm{mg} / \mathrm{kg})$ three times a week for 4 weeks. According to Wei et al., liquiritin isolated from Glycyrrhiza uralensis Fischer. Radix (Leguminosae/Fabaceae/Fabaceae) could circumvent the resistance of cisplatin-based chemotherapy [137]. SGC7901/DDP cells were treated with DDP $(2 \mu \mathrm{g} / \mathrm{ml})$ or liquiritin $(80 \mu \mathrm{M})$ or both for 24 $\mathrm{h}$. The combination greatly suppressed cell proliferation and induced apoptosis, autophagy and G0/G1 phase cell cycle arrest against DDP resistant gastric cancer cells compared to DDP single treatment. Cleavage of caspase-8, caspase-9, caspase- 3 and PARP were activated and increased expression levels of LC-3B and Beclin 1 were observed. Abdelfattah et al. isolated a new alkyl sulfonic acid derivative from the methanolic extract of mycelium of Streptomyces sp. IFM 11694 and named it sulfotanone [138]. Its bioactivity was evaluated for effects on TRAIL-resistance of AGS cells by comparing cell viability in the presence and absence of TRAIL (100 ng/mL). Sulfotanone showed TRAIL-resistance overcoming activity in AGS cells at concentrations of $40 \mu \mathrm{M}$. Bufalin, a traditional Chinese medicine extracted from Venenum bufonis, showed synergetic effects with cisplatin to inhibit proliferation and promote apoptosis of gastric cancer cells SGC-7901, MKN-45, and BGC-823 by diminishing the activation of cisplatin-induced Akt and its downstream molecules under normoxic and hypoxic conditions [139]. Astragalus polysaccharide and apatinib cotreatment were reported to enhance apoptosis compared to apatinib monotherapy [140]. The efficacy of astragalus polysaccharide, an active component extracted from Astragalus mambranaceus Bunge Radix (Leguminosae/Fabaceae/Fabaceae), arises mainly from its ability to inhibit autophagy of apatinib-resistant cells which serves as a survival mechanism. Abdelfattah et al. isolated a new ana-quinonoid tetracene metabolite from the ethyl acetate extract of the culture of marine bacteria Streptomyces sp. EGY1 and named it sharkquinone [141]. Sharkquinone sensitized TRAIL-resistant AGS cells and suggested their potential use in combination with TRAIL against AGS cells. Tanshinone IIA is identified as an interesting agent with potential to treat doxorubicin-resistant gastric cancer cells. [142]. $5 \mu \mathrm{M}$ Tanshinone IIA solution combined with $0.05 \mu \mathrm{g} / \mathrm{mL}$ of doxorubicin showed anticancer effect against doxorubicin-resistant cell lines including SNU-638, SNU668 and SNU-216 and SNU-620. Apoptosis was mainly induced by inhibition of multidrug resistance-associated protein 1 (MRP1). Zuo Jin Wan Formula is a traditional Chinese medicine pill that consists of Coptidis Rhizoma (Ranunculaceae), and Evodiae rutaecarpa Bentham. Fructus (Rutaceae) in the ratio of 6:1. Sun et al. reported that Zuo Jin Wan Formula induced apoptosis in primary DDP-resistant gastric carcinoma cells by stimulating cofilin1 mitochondrial translocation [143]. Translocation of cofilin-1 was mediated by Akt. Accumulation of Bax was observed while expression level of Bcl-2 was decreased. Natural protucts and their target signal is presented in figure 5. 


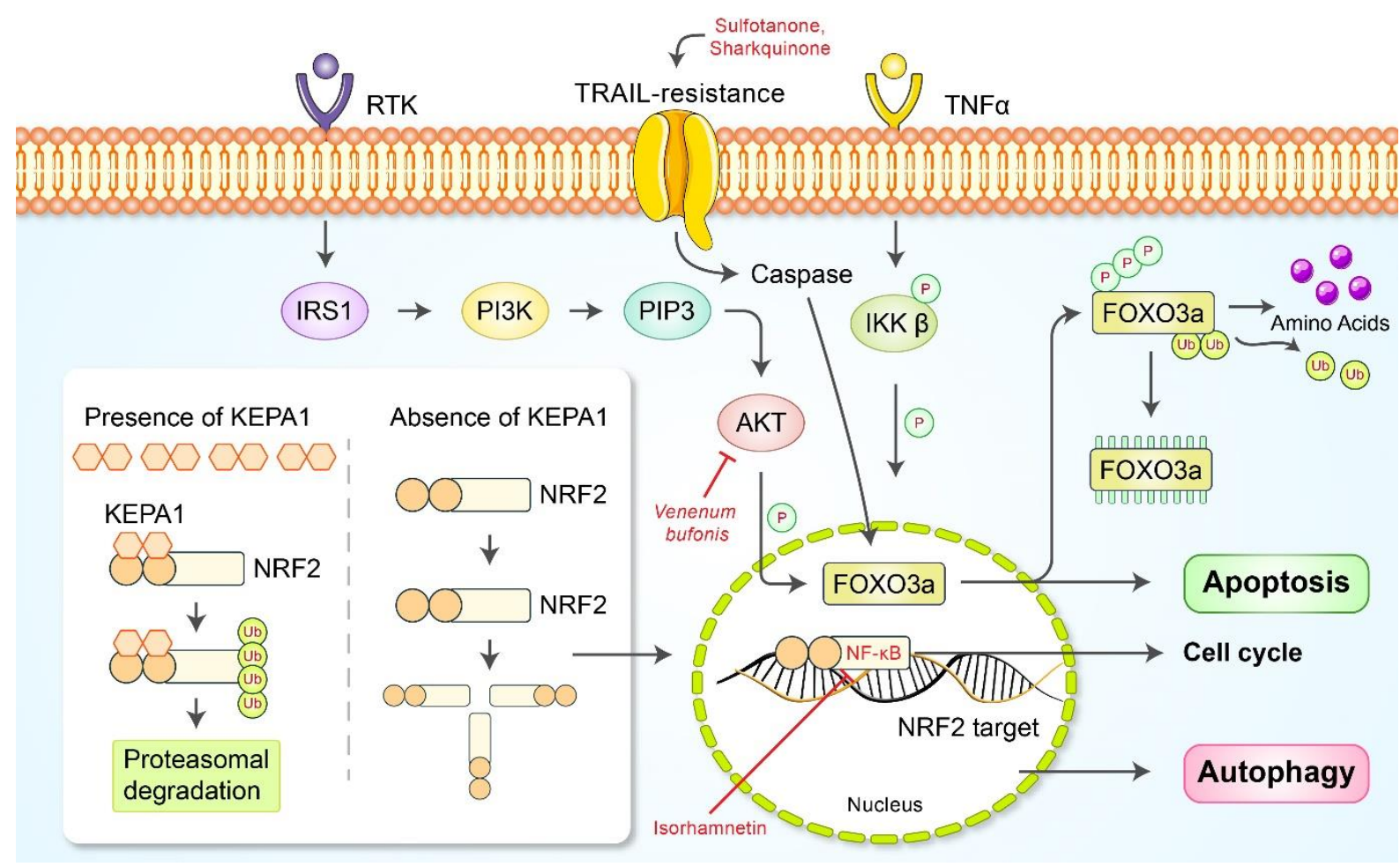

Figure 5. Schematic diagram of resistance signaling pathway. RTK, Receptor tyrosine kinase; IRS1, Insulin receptor substrate 1; PI3K, Phosphoinositide 3-kinases; PIP3, Phosphatidylinositol (3,4,5)-trisphosphate; AKT, Protein kinase B (PKB); FOXO3a, Forkhead box O 3; IKK- $\beta$, Inhibitor of nuclear factor $\kappa \mathrm{B}$ kinase subunit beta; TNF- $\alpha$, Tumor necrosis factor $\alpha$; $\mathrm{Ub}$, Ubiquitin; KEAP1, Kelch-like ECH-associated protein 1; NRF2, Nuclear factor erythroid 2-related factor 2.

Although specific targets vary, most natural products aimed to prevent drug resistance by downregulating Akt and NF- $\mathrm{kB}$ and following pathways (Figure 4). Bufalin from Venenum bufonis and the Zuo Jin Wan formula effectively down-regulated Akt [139, 143]. Similarly, the mineral isorhamnetin from Quercetin inhibited cell viability and prevented drug resistance by downregulating NF- $\mathrm{B}$ [130]. Reduction of drug resistance by natural compounds was demonstrated in other aspects. Pseudolaric acid B from Pseudolarix kaempferi inhibited COX-2, PKC- $\alpha$ and P-gp, factors that promote angiogenesis by cancer cells. Liquirtin from the Glycyrrhiza genus promoted p53, p21 and caspase cleavages while inhibiting cyclin activities. The compound's anti-resistant ability may be focused on apoptotic effects. Other factors such as Bax/Bcl-2 in mitochondria, ERK1/2, MMP2, and PARP are broadly affected by many natural products covered in the review. Each compound, extract or decoction had anti-resistant effects over various aspects of cancer cells when tested in vitro or on animal models.

\section{Limitation and future perspectives of natural products in gastric cancer treatments}

Gastric cancer is known to account for the fifth highest incidence and the fourth highest mortality among all cancers worldwide [1]. Chemotherapy is one of the methods typically used in advanced gastric cancer treatment, but it exerts severe side effects that limit the efficacies and decrease quality of life. Development of therapeutic remedies with less adverse effects and lower chemo-resistance is required. Natural products are emerging as alternative resources to combat gastric carcinoma. While few natural products such as aflatoxins may be noxious or even carcinogenic [144], many phytochemicals with beneficial bioactivity can be successfully selected through deliberate investigations. Therefore, several natural resources obtained from dietary fruits and vegetables were discussed is this review. Curcumin, black currant extract, oligosaccharide isolated from tomato, sulforaphane derived from broccoli, citrus pectin originated from tangerine, grapefruit, 
lemon and orange are good examples. These medicinal resources are still being extensively used in traditional Korean and Chinese medicine. Many natural substances were shown to exhibit multiple effects. The variety is attributed to the structural diversity and multi-target characteristic of natural compounds [145]. Several decoctions were also reported to suppress cancerous cells and tumors comprehensively. In this light, decoctions have the potential to develop as effective therapeutic agents against gastric cancer based on their multi-efficacy and enhancement of efficacy through various methods of post-processing. 
Table 13. Drug resistance-overcoming natural products in vitro.

\begin{tabular}{|c|c|c|c|c|c|c|c|}
\hline Classification & $\begin{array}{l}\text { Compound/ Ex- } \\
\text { tract }\end{array}$ & Source & Experimental Model & Dose; Duration & Efficacy & Mechanism & Reference \\
\hline Flavonoid & Isorhamnetin & Quercetin & $\begin{array}{c}\text { AGS, SNU5, SNU16, } \\
\text { MKN45, NUGC3, } \\
\text { AZ521 }\end{array}$ & $\begin{array}{l}50 \mu \mathrm{M} ; 6,12 \\
24, \text { and } 48 \mathrm{~h}\end{array}$ & Inhibition of the cell viability & $\begin{array}{l}\downarrow N F-\kappa B, p 65, \text { VEGF, } \\
\text { COX-2, MMP-9 }\end{array}$ & {$[136]$} \\
\hline Flavonoid & Liquiritin & $\begin{array}{l}\text { (family: Legu- } \\
\text { minosae/Fa- } \\
\text { baceae/Faba- } \\
\text { ceae) Glycyr- } \\
\text { rhiza ura- } \\
\text { lensis Fischer. } \\
\text { Radix }\end{array}$ & SGC-7901/DDP & $80 \mu \mathrm{M} ; 24 \mathrm{~h}$ & $\begin{array}{l}\text { Potentiation of the suppressive effects of } \\
\text { DDP, induction of G0/G1 cell cycle arrest, } \\
\text { enhancement of apoptosis and autophagy }\end{array}$ & $\begin{array}{l}\uparrow c-\text { caspase- } 8,-9,-3, \text { c- } \\
\text { PARP, LC-3B, Beclin } 1\end{array}$ & {$[137]$} \\
\hline $\begin{array}{l}\text { Organosulfur } \\
\text { compound }\end{array}$ & Sulfotanone & $\begin{array}{l}\text { Streptomyces } \\
\text { IFM } 11694\end{array}$ & AGS & $\begin{array}{c}20,40,80 \mu \mathrm{M} \\
16 \mathrm{~h}\end{array}$ & $\begin{array}{l}\text { Induction of TRAIL resistance-overcoming } \\
\text { activity }\end{array}$ & & {$[138]$} \\
\hline Phytosterol & Bufalin & $\begin{array}{l}\text { Venenum } \\
\text { bufonis }\end{array}$ & $\begin{array}{l}\text { SGC7091, } \\
\text { MKN45, } \\
\text { BGC823 }\end{array}$ & $\begin{array}{l}50,100,200 \\
\mathrm{nM} ; 48 \mathrm{~h}\end{array}$ & $\begin{array}{l}\text { Inhibition of acquired cisplatin resistance } \\
\text { and proliferation } \\
\text { Induction of apoptosis }\end{array}$ & $\downarrow \mathrm{Akt}$ & [139] \\
\hline
\end{tabular}

(family: Leguminosae/Fa-

Polyphenol Astragalus polysaccharide ceae) Astragalus mambranaceus Bung-

eRadix

Synergistic effect of inhibitory effects on cell proliferation, migration, invasion and apoptosis with apatinib $\downarrow$ Akt, MMP-9 
Polyphenol Sharkquinone Streptomyces

Polyphenol Sharkquinone sp. EGY1
AGC

Sensitization of TRAIL-resistant AGC cells

\begin{tabular}{|c|c|c|c|c|c|c|c|}
\hline Terpenoid & Tanshinone IIA & $\begin{array}{c}\text { (family: Lami- } \\
\text { aceae) Salvia } \\
\text { miltiorrhiza } \\
\text { Bunge Radix }\end{array}$ & $\begin{array}{l}\text { SNU-638, SNU-668, } \\
\text { SNU-216, SNU-620 }\end{array}$ & $5 \mu \mathrm{M} ; 24 \mathrm{~h}$ & $\begin{array}{c}\text { Induction of apoptosis, autophagy and cell } \\
\text { cycle arrest }\end{array}$ & $\downarrow \mathrm{MRP} 1$ & {$[142]$} \\
\hline & $\begin{array}{l}\text { Zuo Jin Wan For- } \\
\text { mula }\end{array}$ & $\begin{array}{c}\text { (family: Ra- } \\
\text { nunculaceae) } \\
\text { Coptidis Rhi- } \\
\text { zoma* (fam- }^{*} \text {, } \\
\text { ily: Rutaceae) } \\
\text { Evodiae ru- } \\
\text { taecarpa } \text { Ben- } \\
\text { tham. Fructus }\end{array}$ & SGC7901/DDP & $\begin{array}{r}20,50,100 \\
\mu \mathrm{g} / \mathrm{mL} ; 48 \mathrm{~h}\end{array}$ & Increase of chemosensitivity & $\begin{array}{c}\uparrow \text { cofilin-1, Bax } \\
\downarrow \text { Akt, Bcl-2 }\end{array}$ & {$[143]$} \\
\hline
\end{tabular}

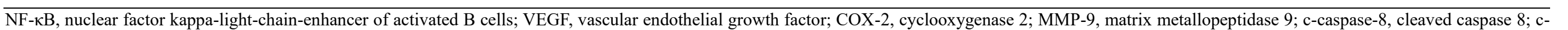

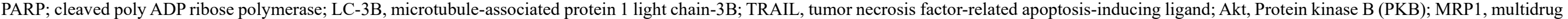

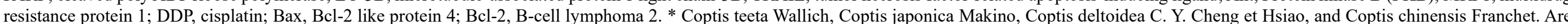
called Coptidis Rhizoma. The study did not mention which species was used.

Table 14. Drug resistance-overcoming natural products in vivo.

\begin{tabular}{|c|c|c|c|c|c|c|c|}
\hline Classification & $\begin{array}{l}\text { Compound/ Ex- } \\
\text { tract }\end{array}$ & Source & Experimental Model & Dose; Duration & Efficacy & Mechanism & Reference \\
\hline Flavonoid & Isorhamnetin & Quercetin & $\begin{array}{l}\text { Athymic nu/nu mice / } \\
\text { SNU-5 }\end{array}$ & $\begin{array}{l}1 \mathrm{mg} / \mathrm{kg} ; 4 \\
\text { weeks }\end{array}$ & Enhancement of the efficacy of capecitabine & $\begin{array}{l}\downarrow \text { NF- } \kappa B, \text { VEGF, COX- } \\
\text { 2, MMP-9 }\end{array}$ & [136] \\
\hline
\end{tabular}

NF-кB, nuclear factor kappa-light-chain-enhancer of activated B cells; VEGF, vascular endothelial growth factor; COX-2, cyclooxygenase 2; MMP-9, matrix metallopeptidase 9. 
While there have been similar reviews highlighting the anti-neoplastic efficacies of natural products or phytochemicals, few of them are written with regards to the chemical classification of each bioactive compound [146]. This review is not only a simple compilation of previous in vitro studies testing natural products on gastric cancer but goes as far as to systematically organizing previous works depending on each cancer related pathways, namely, apoptosis, autophagy, metastasis, drug-resistant capability and more. Studies grouped into each pathway were then organized according to the phytochemical classification of the active compounds. As there is currently no golden standard for classifying phytochemicals, we adopted a comprehensive and clear method previously demonstrated in a literature highlighting the efficacies of natural products on gastrointestinal diseases [147]. This will help researchers rule out or select appropriate candidate species of natural products for further studies. One limitation of this review is that it only included studies published from 2014 to 2020. Also, clinical trials were excluded to focus on laboratory experiments highlighting specific biological pathways. Several investigations were insufficient to elucidate anti-cancer mechanisms at molecular levels. They were generally focusing on the cytotoxicity of the chemicals or reporting newly discovered compounds, which makes incisive research burdensome. By and large, more than half of the studies only carried out experiments in vitro. More in vivo studies are recommended to bridge the advance to clinical trials and therapeutic use. Decoctions and extracts proven safe for human should go under well designed clinical trials to prove their efficacy in the evidence-based medicine perspective.

Natural products are indeed effective in the single compound to single target mechanistic perspective; however, it is worth highlighting the complex interactions between many compounds. While the importance of studying the interactions between multi-compound natural products and other drugs was previously highlighted in many literatures, it is also important to further investigate the interactions between different natural products, including herbal medicines in a biochemical manner [148]. A systemic approach with focus on structural similarities of several phytochemical compounds and human metabolites is a potential way of clearly highlighting the efficacies of multi compound drugs. Furthermore, the fact that current drug interaction analytical methodology for novel multi-targeted agents closely resembles the Kun-Shin-Choa-Sa in many perspectives makes it more intriguing to apply this principle in modern drug studies $[149,150]$.

\section{Conclusions}

In this review, we summed up seventy-six studies considering natural products that have anti-cancer efficacy against gastric cancer. Natural products mainly induced cell death by apoptosis and autophagy, cell cycle arrest, inhibit angiogenesis and metastasis, and circumvent chemo-resistance against stomach cancer cells in vitro or in vivo through various molecular mechanisms. Several compounds, extracts and decoctions showed multiple efficacies, attributed to structural complexity and multiple target pathways and proteins of natural products. Thus, natural substances implicate possibilities of being used in nutrition or medications which may lead to novel discoveries in alternative medicine in cancer treatment. This review would provide data for future research and clinical trials to develop novel drugs from natural products for gastric cancer treatment. 
Author Contributions: Methodology, S.Y.K.; validation, S.Y.K., D.H., S.S. and J.P.; investigation, S.Y.K., D.H., S.S., M.K. and J.P.; writing - original draft preparation, S.Y.K., D.H., S.S., M.K. and J.P.; writing-review and editing, S.Y.K., D.H. and B.K.; visualization, S.S, J.P. and D.H.; supervision, B.K.; project administration, B.K.; M.H.R. figure dwawing and modification; M.A.R. editing and reviewing; funding acquisition, B.K. All authors have read and agreed to the published version of the manuscript.

Funding: This research was supported by Basic Science Research Program through the National Research Foundation of Korea (NRF) funded by the Ministry of Education (NRF2020R1I1A2066868), the National Research Foundation of Korea (NRF) grant funded by the Korea government (MSIT) (No. 2020R1A5A2019413), a grant of the Korea Health Technology R\&D Project through the Korea Health Industry Development Institute (KHIDI), funded by the Ministry of Health \& Welfare, Republic of Korea (grant number : HF20C0116), and a grant of the Korea Health Technology R\&D Project through the Korea Health Industry Development Institute (KHIDI), funded by the Ministry of Health \& Welfare, Republic of Korea (grant number : HF20C0038).

Conflicts of Interest: The authors declare no conflict of interest. 


\section{References}

1. Sung, H., et al., Global cancer statistics 2020: GLOBOCAN estimates of incidence and mortality worldwide for 36 cancers in 185 countries. CA: A Cancer Journal for Clinicians, 2021.

2. $\mathrm{Hu}, \mathrm{B}$., et al., Gastric cancer: Classification, histology and application of molecular pathology. J Gastrointest Oncol, 2012. 3(3): p. 251-61

3. Crew, K.D. and A.I. Neugut, Epidemiology of gastric cancer. World J Gastroenterol, 2006. 12(3): p. 354-62.

4. Rawla, P. and A. Barsouk, Epidemiology of gastric cancer: global trends, risk factors and prevention. Prz Gastroenterol, 2019. 14(1): p. 26-38.

5. $\quad$ Ajani, J.A., et al., Gastric Cancer, Version 3.2016, NCCN Clinical Practice Guidelines in Oncology. J Natl Compr Canc Netw, 2016. 14(10): p. 1286-1312.

6. Ronellenfitsch, U., et al., Perioperative chemo(radio)therapy versus primary surgery for resectable adenocarcinoma of the stomach, gastroesophageal junction, and lower esophagus. Cochrane Database Syst Rev, 2013(5): p. Cd008107.

7. Diaz-Nieto, R., R. Orti-Rodríguez, and M. Winslet, Post-surgical chemotherapy versus surgery alone for resectable gastric cancer. Cochrane Database Syst Rev, 2013(9): p. Cd008415.

8. Oba, K., et al., Disease-free survival as a surrogate for overall survival in adjuvant trials of gastric cancer: a meta-analysis. J Natl Cancer Inst, 2013. 105(21): p. 1600-7.

9. Gibson, R.J. and D.M. Keefe, Cancer chemotherapy-induced diarrhoea and constipation: mechanisms of damage and prevention strategies. Support Care Cancer, 2006. 14(9): p. 890-900.

10. Staff, N.P., et al., Chemotherapy-induced peripheral neuropathy: A current review. Annals of Neurology, 2017. 81(6): p. $772-781$

11. Yang, W., et al., Molecular mechanisms and theranostic potential of miRNAs in drug resistance of gastric cancer. Expert Opin Ther Targets, 2017. 21(11): p. 1063-1075.

12. Singh, N., et al., Inflammation and cancer. Ann Afr Med, 2019. 18(3): p. 121-126.

13. Lu, B. and M. Li, Helicobacter pylori eradication for preventing gastric cancer. World J Gastroenterol, 2014. 20(19): p. 5660-5.

14. Sokolova, O. and M. Naumann, NF-kappaB Signaling in Gastric Cancer. Toxins (Basel), 2017. 9(4).

15. Lee, H., A.J. Jeong, and S.K. Ye, Highlighted STAT3 as a potential drug target for cancer therapy. BMB Rep, 2019. 52(7): p. 415-423.

16. Naylor, M.S., et al., Tumor necrosis factor and its receptors in human ovarian cancer. Potential role in disease progression. J Clin Invest, 1993. 91(5): p. 2194-206.

17. Wang, X. and Y. Lin, Tumor necrosis factor and cancer, buddies or foes? Acta Pharmacol Sin, 2008. 29(11): p. $1275-88$.

18. Bastos, J., et al., Dietary patterns and gastric cancer in a Portuguese urban population. Int J Cancer, 2010. 127(2): p. 433-41.

19. Nagata, C., et al., A prospective cohort study of soy product intake and stomach cancer death. Br J Cancer, 2002. 87(1): p. 31-6.

20. Steevens, J., et al., Vegetables and fruits consumption and risk of esophageal and gastric cancer subtypes in the Netherlands Cohort Study. Int J Cancer, 2011. 129(11): p. 2681-93.

21. Mao, Q.Q., et al., Phytochemicals for the Prevention and Treatment of Gastric Cancer: Effects and Mechanisms. Int J Mol Sci, 2020. 21(2).

22. Xu, J., et al., Anticancer effect of berberine based on experimental animal models of various cancers: a systematic review and meta-analysis. BMC Cancer, 2019. 19(1): p. 589.

23. Hassanalilou, T., S. Ghavamzadeh, and L. Khalili, Curcumin and Gastric Cancer: a Review on Mechanisms of Action. J Gastrointest Cancer, 2019. 50(2): p. 185-192.

24. Dutta, S., et al., Natural products: An upcoming therapeutic approach to cancer. Food Chem Toxicol, 2019. 128: p. 240255.

25. Kim, H.-J., J.-Y. Um, and Y.-K. Kim, Glutathione S-transferase gene polymorphism in Korean subjects with gastric and colorectal cancer. Oriental Pharmacy and Experimental Medicine, 2012. 12(4): p. 307-312.

26. Mann, J., Natural products in cancer chemotherapy: past, present and future. Nat Rev Cancer, 2002. 2(2): p. 143-8.

27. Wang, L., et al., Anticancer potential of bioactive peptides from animal sources (Review). Oncol Rep, 2017. 38(2): p. 637-651.

28. Gras, M., et al., Use of Complementary and Alternative Medicines among Cancer Patients: A Single-Center Study. Oncology, 2019. 97(1): p. 18-25.

29. Li, X., et al., Traditional Chinese medicine in cancer care: a review of controlled clinical studies published in chinese. PLoS One, 2013. 8(4): p. e60338.

30. Wode, K., et al., Cancer patients' use of complementary and alternative medicine in Sweden: a cross-sectional study. BMC Complement Altern Med, 2019. 19(1): p. 62.

31. Kristoffersen, A.E., et al., Use of traditional and complementary medicine among Norwegian cancer patients in the seventh survey of the Tromso study. BMC Complement Altern Med, 2019. 19(1): p. 341. 
32. Sun, Y. and Z.L. Peng, Programmed cell death and cancer. Postgrad Med J, 2009. 85(1001): p. 134-40.

33. Greco, G., E. Catanzaro, and C. Fimognari, Natural Products as Inducers of Non-Canonical Cell Death: A Weapon against Cancer. Cancers (Basel), 2021. 13(2).

34. Hosseini, F.S., et al., Evaluating of Induction of Apoptosis by Cornus mass L. Extract in the Gastric Carcinoma Cell Line (AGS). Asian Pac J Cancer Prev, 2019. 20(1): p. 123-130.

35. Ghasemi, S., et al., Cytotoxic effects of Urtica dioica radix on human colon (HT29) and gastric (MKN45) cancer cells mediated through oxidative and apoptotic mechanisms. Cell Mol Biol (Noisy-le-grand), 2016. 62(9): p. 90-96.

36. Elmore, S., Apoptosis: a review of programmed cell death. Toxicologic pathology, 2007. 35(4): p. $495-516$.

37. Redza-Dutordoir, M. and D.A. Averill-Bates, Activation of apoptosis signalling pathways by reactive oxygen species. Biochim Biophys Acta, 2016. 1863(12): p. 2977-2992.

38. Yang, Y., et al., Integration of microRNA-mRNA profiles and pathway analysis of plant isoquinoline alkaloid berberine in SGC-7901 gastric cancers cells. Drug Des Devel Ther, 2018. 12: p. 393-408.

39. Wu, J., et al., Cyclovirobuxine D Inhibits Cell Proliferation and Induces Mitochondria-Mediated Apoptosis in Human Gastric Cancer Cells. Molecules, 2015. 20(11): p. 20659-68.

40. Cui, F., et al., Grifola frondosa Glycoprotein GFG-3a Arrests S phase, Alters Proteome, and Induces Apoptosis in Human Gastric Cancer Cells. Nutr Cancer, 2016. 68(2): p. 267-79.

41. Kong, G.M., et al., Melittin induces human gastric cancer cell apoptosis via activation of mitochondrial pathway. World J Gastroenterol, 2016. 22(11): p. 3186-95.

42. Wu, D., et al., Anti-tumor effects of phenolic alkaloids of menispermum dauricum on gastric cancer in vivo and in vitro. $\mathrm{J}$ Cancer Res Ther, 2018. 14(Supplement): p. S505-S511.

43. Zhang, X.Z., et al., Synergistic inhibitory effect of berberine and d-limonene on human gastric carcinoma cell line MGC803. J Med Food, 2014. 17(9): p. 955-62.

44. Lu, X., et al., Anti-proliferation effects of trifolirhizin on MKN45 cells and possible mechanism. Oncol Rep, 2016. 36(5): p. $2785-2792$.

45. Qian, J., et al., Ginsenoside-Rh2 Inhibits Proliferation and Induces Apoptosis of Human Gastric Cancer SGC-7901 Side Population Cells. Asian Pac J Cancer Prev, 2016. 17(4): p. 1817-21.

46. Su, C.C., Tanshinone IIA inhibits gastric carcinoma AGS cells through increasing $p$-p38, $p$-JNK and $p 53$ but reducing $p$ ERK, CDC2 and cyclin B1 expression. Anticancer Res, 2014. 34(12): p. 7097-110.

47. Mansingh, D.P., et al., [6]-Gingerol-induced cell cycle arrest, reactive oxygen species generation, and disruption of mitochondrial membrane potential are associated with apoptosis in human gastric cancer (AGS) cells. J Biochem Mol Toxicol, 2018. 32(10): p. e22206.

48. Zhu, H., et al., Anticancer effect of 2,7-dihydroxy-3-methylanthraquinone on human gastric cancer SGC-7901 cells in vitro and in vivo. Pharm Biol, 2016. 54(2): p. 285-92.

49. Luo, Y., et al., A flavonoid compound from Chrysosplenium nudicaule inhibits growth and induces apoptosis of the human stomach cancer cell line SGC-7901. Pharm Biol, 2016. 54(7): p. 1133-9.

50. Sun, Q., et al., Curcumin inhibits cell growth and induces cell apoptosis through upregulation of miR-33b in gastric cancer. Tumour Biol, 2016. 37(10): p. 13177-13184.

51. Liu, L., et al., Hydroxysafflor-Yellow A Induces Human Gastric Carcinoma BGC-823 Cell Apoptosis by Activating Peroxisome Proliferator-Activated Receptor Gamma (PPARgamma). Med Sci Monit, 2018. 24: p. 803-811.

52. Zhou, W., et al., Kurarinone Synergizes TRAIL-Induced Apoptosis in Gastric Cancer Cells. Cell Biochem Biophys, 2015. 72(1): p. 241-9.

53. Wu, J., et al., Licochalcone A suppresses hexokinase 2-mediated tumor glycolysis in gastric cancer via downregulation of the Akt signaling pathway. Oncol Rep, 2018. 39(3): p. 1181-1190.

54. Zhang, W., et al., Effects of ophiopogonin B on the proliferation and apoptosis of SGC7901 human gastric cancer cells. Mol Med Rep, 2016. 13(6): p. 4981-6.

55. Xu, M., et al., Anticancer Activity of Phloretin Against Human Gastric Cancer Cell Lines Involves Apoptosis, Cell Cycle Arrest, and Inhibition of Cell Invasion and JNK Signalling Pathway. Med Sci Monit, 2018. 24: p. 6551-6558.

56. Akbari Asl, E., et al., Apoptotic Effects of Linum album Extracts on AGS Human Gastric Adenocarcinoma Cells and ZNF703 Oncogene Expression. Asian Pac J Cancer Prev, 2018. 19(10): p. 2911-2916.

57. Tsai, T.C., et al., 7-Acetylsinumaximol B Induces Apoptosis and Autophagy in Human Gastric Carcinoma Cells through Mitochondria Dysfunction and Activation of the PERK/eIF2alpha/ATF4/CHOP Signaling Pathway. Mar Drugs, 2018.

16(4).

58. Cheng, Q.L., et al., CRA(Crosolic Acid) isolated from Actinidia valvata Dunn.Radix induces apoptosis of human gastric cancer cell line BGC823 in vitro via down-regulation of the NF-kappaB pathway. Food Chem Toxicol, 2017. 105: $\mathrm{p}$. 475-485.

59. Jung, E.B., et al., Curcuzedoalide contributes to the cytotoxicity of Curcuma zedoaria rhizomes against human gastric cancer AGS cells through induction of apoptosis. J Ethnopharmacol, 2018. 213: p. 48-55.

60. Zhang, D., et al., Deacetylisovaltratum disrupts microtubule dynamics and causes G2/M-phase arrest in human gastric cancer cells in vitro. Acta Pharmacol Sin, 2016. 37(12): p. 1597-1605. 
61. Li, P., et al., Elemene Induces Apoptosis of Human Gastric Cancer Cell Line BGC-823 via Extracellular Signal-Regulated Kinase (ERK) 1/2 Signaling Pathway. Med Sci Monit, 2017. 23: p. 809-817.

62. Wu, Z. and Y. Li, Grifolin exhibits anti-cancer activity by inhibiting the development and invasion of gastric tumor cells. Oncotarget, 2017. 8(13): p. 21454-21460.

63. Liao, K.F., et al., Anti-Cancer Effects of Radix Angelica Sinensis (Danggui) and N-Butylidenephthalide on Gastric Cancer: Implications for REDD1 Activation and mTOR Inhibition. Cell Physiol Biochem, 2018. 48(6): p. 2231-2246.

64. Lyu, Z.K., et al., Paeonol exerts potential activities to inhibit the growth, migration and invasion of human gastric cancer BGC823 cells via downregulating MMP2 and MMP9. Mol Med Rep, 2017. 16(5): p. 7513-7519.

65. Yu, F., et al., Pseudolaric acid B circumvents multidrug resistance phenotype in human gastric cancer SGC7901/ADR cells by downregulating Cox-2 and P-gp expression. Cell Biochem Biophys, 2015. 71(1): p. 119-26.

66. Kang, S.H., et al., Anticancer Effect of Thymol on AGS Human Gastric Carcinoma Cells. J Microbiol Biotechnol, 2016. 26(1): p. 28-37.

67. Wang, G., et al., Toosendanin suppresses oncogenic phenotypes of human gastric carcinoma SGC7901 cells partly via miR200amediated downregulation of beta-catenin pathway. Int J Oncol, 2017. 51(5): p. 1563-1573.

68. Zhou, Q., et al., Toosendanin induces caspase-dependent apoptosis through the p38 MAPK pathway in human gastric cancer cells. Biochem Biophys Res Commun, 2018. 505(1): p. 261-266.

69. Zhang, L., et al., Arsenic sulfide, the main component of realgar, a traditional Chinese medicine, induces apoptosis of gastric cancer cells in vitro and in vivo. Drug Des Devel Ther, 2015. 9: p. 79-92.

70. Kapoor, S. and S.M. Dharmesh, Pectic Oligosaccharide from tomato exhibiting anticancer potential on a gastric cancer cell line: Structure-function relationship. Carbohydr Polym, 2017. 160: p. 52-61.

71. Wang, X.P., et al., Anti-tumor bioactivities of curcumin on mice loaded with gastric carcinoma. Food Funct, 2017. 8(9): p. 3319-3326.

72. Li, L., et al., Periplocin Extracted from Cortex Periplocae Induced Apoptosis of Gastric Cancer Cells via the ERK1/2EGR1 Pathway. Cell Physiol Biochem, 2016. 38(5): p. 1939-51.

73. Pan, H., et al., Esculetin induces apoptosis in human gastric cancer cells through a cyclophilin D-mediated mitochondrial permeability transition pore associated with ROS. Chem Biol Interact, 2015. 242: p. 51-60.

74. Yang, Y., et al., Acetonic extracts of the endolichenic fungus EL002332 isolated from Endocarpon pusillum exhibits anticancer activity in human gastric cancer cells. Phytomedicine, 2018. 40: p. 106-115.

75. Liu, B. and Z. Li, Black Currant (Ribes nigrum L.) Extract Induces Apoptosis of MKN-45 and TE-1 Cells Through MAPK- and PI3K/Akt-Mediated Mitochondrial Pathways. J Med Food, 2016. 19(4): p. 365-73.

76. $\quad \mathrm{Fu}, \mathrm{Z}$., et al., Euphorbia lunulata extract acts on multidrug resistant gastric cancer cells to inhibit cell proliferation, migration and invasion, arrest cell cycle progression, and induce apoptosis. J Ethnopharmacol, 2018. 212: p. 8-17.

77. Xie, H., et al., Ethanolic extract of Cordyceps cicadae exerts antitumor effect on human gastric cancer SGC-7901 cells by inducing apoptosis, cell cycle arrest and endoplasmic reticulum stress. J Ethnopharmacol, 2019. 231: p. $230-240$.

78. Hao, G., et al., Smilax glabra Roxb targets Akt(p-Thr308) and inhibits Akt-mediated signaling pathways in SGC7901 cells. J Drug Target, 2016. 24(6): p. 557-65.

79. Wang, Z., et al., Astragalus extract inhibits proliferation but enhances apoptosis in gastric cancer. Pak J Pharm Sci, 2016. 29(5): p. 1473-1482.

80. Jeong, H.J., et al., Inhibitory effects of Saururus chinensis and its components on stomach cancer cells. Phytomedicine, 2015. 22(2): p. 256-61.

81. Kim, J.S., et al., Radix Sophorae Flavescentis inhibits proliferation and induces apoptosis of AGS human gastric cancer cells. Mol Med Rep, 2019. 19(3): p. 1911-1918.

82. Wang, H., et al., Swertia mussotii extracts induce mitochondria-dependent apoptosis in gastric cancer cells. Biomed Pharmacother, 2018. 104: p. 603-612.

83. Kustiawan, P.M., et al., Propolis from the Stingless Bee Trigona incisa from East Kalimantan, Indonesia, Induces In Vitro Cytotoxicity and Apoptosis in Cancer Cell lines. Asian Pac J Cancer Prev, 2015. 16(15): p. 6581-9.

84. Kim, H.S., et al., Schizandra chinensis extracts induce apoptosis in human gastric cancer cells via JNK/p38 MAPK activation and the ROS-mediated/mitochondria-dependent pathway. Pharm Biol, 2015. 53(2): p. 212-9.

85. Zhang, L., et al., Trichloromethane fraction of Incarvillea compacta induces lytic cytotoxicity and apoptosis in EpsteinBarr virus-positive gastric cancer AGS cells. BMC Complement Altern Med, 2016. 16: p. 344.

86. Fu, Z.Y., et al., Apoptosis of human gastric carcinoma cells induced by Euphorbia esula latex. World J Gastroenterol, 2016. 22(13): p. 3564-72.

87. Ngabire, D., et al., Induction of apoptosis and G1 phase cell cycle arrest by Aster incisus in AGS gastric adenocarcinoma cells. Int J Oncol, 2018. 53(5): p. 2300-2308.

88. Li, J., et al., Chinese patent medicine (Jinlong Capsule) for gastric cancer: Protocol for a systematic review and metaanalysis. Medicine (Baltimore), 2020. 99(23): p. e20532.

89. Li, D., et al., Jinlong Capsule (JLC) inhibits proliferation and induces apoptosis in human gastric cancer cells in vivo and in vitro. Biomed Pharmacother, 2018. 107: p. 738-745. 
90. Xu, J., et al., Xiao Tan He Wei Decoction reverses MNNG-induced precancerous lesions of gastric carcinoma in vivo and vitro: Regulation of apoptosis through NF-kappaB pathway. Biomed Pharmacother, 2018. 108: p. 95-102.

91. Deng, H.X., et al., Yangzheng Sanjie decoction regulates proliferation and apoptosis of gastric cancer cells by enhancing let-7a expression. World J Gastroenterol, 2017. 23(30): p. 5538-5548.

92. Yim, N.H., et al., Fermented So-Cheong-Ryong-Tang (FCY) induces apoptosis via the activation of caspases and the regulation of MAPK signaling pathways in cancer cells. BMC Complement Altern Med, 2015. 15: p. 336.

93. Feng, Y., et al., The machinery of macroautophagy. Cell Research, 2014. 24(1): p. 24-41.

94. Rahman, M.A. and H. Rhim, Therapeutic implication of autophagy in neurodegenerative diseases. BMB Rep, 2017. 50(7): p. 345-354.

95. Kang, R., et al., The Beclin 1 network regulates autophagy and apoptosis. Cell Death \& Differentiation, 2011. 18(4): p. 571-580.

96. Rahman, M.A., et al., Phytochemicals as a Complement to Cancer Chemotherapy: Pharmacological Modulation of the Autophagy-Apoptosis Pathway. Frontiers in Pharmacology, 2021. 12.

97. Onorati, A.V., et al., Targeting autophagy in cancer. Cancer, 2018. 124(16): p. 3307-3318.

98. Mandhair, H.K., et al., Molecular modulation of autophagy: New venture to target resistant cancer stem cells. World J Stem Cells, 2020. 12(5): p. 303-322.

99. Rahman, M.A., et al., Antioxidant Compound, Oxyresveratrol, Inhibits APP Production through the AMPK/ULK1/mTOR-Mediated Autophagy Pathway in Mouse Cortical Astrocytes. Antioxidants, 2021. 10(3).

100. Polson, H.E.J., et al., Mammalian Atg18 (WIPI2) localizes to omegasome-anchored phagophores and positively regulates LC3 lipidation. Autophagy, 2010. 6(4): p. 506-522.

101. Tanida, I., T. Ueno, and E. Kominami, LC3 conjugation system in mammalian autophagy. The International Journal of Biochemistry \& Cell Biology, 2004. 36(12): p. 2503-2518.

102. Hannah, et al., WIPI2 Links LC3 Conjugation with PI3P, Autophagosome Formation, and Pathogen Clearance by Recruiting Atg 12-5-16L1. Molecular Cell, 2014. 55(2): p. 238-252.

103. Rahman, M.A., et al., Molecular Insights into the Multifunctional Role of Natural Compounds: Autophagy Modulation and Cancer Prevention. Biomedicines, 2020. 8(11).

104. Rahman, M.A., et al., Pharmacological Inhibition of O-GlcNAc Transferase Promotes mTOR-Dependent Autophagy in Rat Cortical Neurons. Brain Sciences, 2020. 10(12).

105. Song, J., et al., Rottlerin promotes autophagy and apoptosis in gastric cancer cell lines. Mol Med Rep, 2018. 18(3): p. 2905-2913.

106. Chun, J., M. Kang, and Y.S. Kim, A triterpenoid saponin from Adenophora triphylla var. japonica suppresses the growth of human gastric cancer cells via regulation of apoptosis and autophagy. Tumour Biol, 2014. 35(12): p. 12021-30.

107. Kwon, Y.H., et al., Morus alba Accumulates Reactive Oxygen Species to Initiate Apoptosis via FOXO-Caspase 3-Dependent Pathway in Neuroblastoma Cells. Molecules and Cells, 2015. 38(7): p. 630-637.

108. Rahman, M.A., et al., Oxyresveratrol activates parallel apoptotic and autophagic cell death pathways in neuroblastoma cells. Biochim Biophys Acta Gen Subj, 2017. 1861(2): p. 23-36.

109. Chen, X., et al., Suppression of gastric cancer by extract from the tuber of amorphophallus konjac via induction of apoptosis and autophagy. Oncol Rep, 2017. 38(2): p. 1051-1058.

110. Chen, P.P., et al., Kangfuxin promotes apoptosis of gastric cancer cells through activating ERstress and autophagy. Mol Med Rep, 2017. 16(6): p. 9043-9050.

111. Rahman, M.A., N.H. Kim, and S.O. Huh, Cytotoxic effect of gambogic acid on SH-SY5Y neuroblastoma cells is mediated by intrinsic caspase-dependent signaling pathway. Mol Cell Biochem, 2013. 377(1-2): p. 187-96.

112. Rahman, M.A., et al., Induction of apoptosis by Dioscorea nipponica Makino extracts in human SH-SY5Y neuroblastoma cells via mitochondria-mediated pathway. Animal Cells and Systems, 2014. 18(1): p. 41-51.

113. Rahman, M.A., J.S. Hong, and S.O. Huh, Antiproliferative properties of Saussurea lappa Clarke root extract in SH-SY5Y neuroblastoma cells via intrinsic apoptotic pathway. Animal Cells and Systems, 2015. 19(2): p. 119-126.

114. Mrakovcic, M. and L.F. Fröhlich, p53-Mediated Molecular Control of Autophagy in Tumor Cells. Biomolecules, 2018. 8(2): p. 14.

115. Cao, Y., et al., Forty-year journey of angiogenesis translational research. Sci Transl Med, 2011. 3(114): p. 114rv3.

116. Wang, Z., et al., Broad targeting of angiogenesis for cancer prevention and therapy. Seminars in cancer biology, 2015. 35 Suppl(Suppl): p. S224-S243.

117. Zhang, L., et al., Arsenic Trioxide Suppressed Migration and Angiogenesis by Targeting FOXO3a in Gastric Cancer Cells. Int J Mol Sci, 2018. 19(12).

118. Yan, B., et al., Xiaotan Sanjie decoction attenuates tumor angiogenesis by manipulating Notch-1-regulated proliferation of gastric cancer stem-like cells. World J Gastroenterol, 2014. 20(36): p. 13105-18.

119. Shi, J., Y. Lu, and P. Wei, Xiaotan Sanjie decoction inhibits angiogenesis in gastric cancer through Interleukin-8-linked regulation of the vascular endothelial growth factor pathway. J Ethnopharmacol, 2016. 189: p. 230-7.

120. Huang, W., et al., Potent anti-angiogenic component in Croton crassifolius and its mechanism of action. J Ethnopharmacol, 2015. 175: p. 185-91. 
121. Suhail, Y., et al., Systems Biology of Cancer Metastasis. Cell systems, 2019. 9(2): p. 109-127.

122. Wen, Z., et al., Evodiamine, a novel inhibitor of the Wnt pathway, inhibits the self-renewal of gastric cancer stem cells. Int J Mol Med, 2015. 36(6): p. 1657-63.

123. Kiani, S., et al., Purified sulforaphane from broccoli (Brassica oleracea var. italica) leads to alterations of CDX1 and CDX2 expression and changes in miR-9 and miR-326 levels in human gastric cancer cells. Gene, 2018. 678: p. 115-123.

124. Zhang, X.R., et al., Isoliquiritigenin inhibits proliferation and metastasis of MKN28 gastric cancer cells by suppressing the PI3K/AKT/mTOR signaling pathway. Mol Med Rep, 2018. 18(3): p. 3429-3436.

125. Liu, W., et al., Dehydroeffusol effectively inhibits human gastric cancer cell-mediated vasculogenic mimicry with low toxicity. Toxicol Appl Pharmacol, 2015. 287(2): p. 98-110.

126. Chen, F., et al., Baicalein inhibits migration and invasion of gastric cancer cells through suppression of the TGF-beta signaling pathway. Mol Med Rep, 2014. 10(4): p. 1999-2003.

127. Dai, L., G. Wang, and W. Pan, Andrographolide Inhibits Proliferation and Metastasis of SGC7901 Gastric Cancer Cells. Biomed Res Int, 2017. 2017: p. 6242103.

128. Wang, S., et al., Chemoprevention of Low-Molecular-Weight Citrus Pectin (LCP) in Gastrointestinal Cancer Cells. Int J Biol Sci, 2016. 12(6): p. 746-56.

129. Zhu, Y., et al., Antimetastatic effects of Celastrus orbiculatus on human gastric adenocarcinoma by inhibiting epithelialmesenchymal transition and NF-kappaB/snail signaling pathway. Integr Cancer Ther, 2015. 14(3): p. 271-81.

130. Zhu, Y., et al., Research on the efficacy of Celastrus Orbiculatus in suppressing TGF-betal-induced epithelial-mesenchymal transition by inhibiting HSP27 and TNF-alpha-induced NF-kappa B/Snail signaling pathway in human gastric adenocarcinoma. BMC Complement Altern Med, 2014. 14: p. 433.

131. Wang, X., et al., Glucose-regulated protein 78 is essential for cardiac myocyte survival. Cell Death Differ, 2018.

132. Tsering, J. and X. Hu, Triphala Suppresses Growth and Migration of Human Gastric Carcinoma Cells In Vitro and in a Zebrafish Xenograft Model. Biomed Res Int, 2018. 2018: p. 7046927.

133. Zhu, X., et al., Traditional Chinese medicine Jianpi Bushen therapy suppresses the onset of pre-metastatic niche in a murine model of spontaneous lung metastasis. Biomed Pharmacother, 2017. 86: p. 434-440.

134. Vasan, N., J. Baselga, and D.M. Hyman, A view on drug resistance in cancer. Nature, 2019. 575(7782): p. $299-309$.

135. Rossiter, S.E., M.H. Fletcher, and W.M. Wuest, Natural Products as Platforms To Overcome Antibiotic Resistance. Chem Rev, 2017. 117(19): p. 12415-12474.

136. Manu, K.A., et al., Isorhamnetin augments the anti-tumor effect of capecitabine through the negative regulation of NFkappaB signaling cascade in gastric cancer. Cancer Lett, 2015. 363(1): p. 28-36.

137. Wei, F., et al., Liquiritin induces apoptosis and autophagy in cisplatin (DDP)-resistant gastric cancer cells in vitro and xenograft nude mice in vivo. Int J Oncol, 2017. 51(5): p. 1383-1394.

138. Abdelfattah, M.S., et al., Sulfotanone, a new alkyl sulfonic acid derivative from Streptomyces sp. IFM 11694 with TRAIL resistance-overcoming activity. J Nat Med, 2016. 70(2): p. 266-70.

139. Zhao, H., et al., Bufalin reverses intrinsic and acquired drug resistance to cisplatin through the AKT signaling pathway in gastric cancer cells. Mol Med Rep, 2016. 14(2): p. 1817-22.

140. $\quad \mathrm{Wu}$, J., et al., Astragalus polysaccharide enhanced antitumor effects of Apatinib in gastric cancer AGS cells by inhibiting AKT signalling pathway. Biomed Pharmacother, 2018. 100: p. 176-183.

141. Abdelfattah, M.S., et al., Sharkquinone, a new ana-quinonoid tetracene derivative from marine-derived Streptomyces sp. EGY1 with TRAIL resistance-overcoming activity. J Nat Med, 2017. 71(3): p. 564-569.

142. $\mathrm{Xu}, \mathrm{Z}$., et al., Potentiation of the anticancer effect of doxorubicinin drug-resistant gastric cancer cells by tanshinone IIA. Phytomedicine, 2018. 51: p. 58-67.

143. Sun, M.Y., et al., The Zuo Jin Wan Formula increases chemosensitivity of human primary gastric cancer cells by AKT mediated mitochondrial translocation of cofilin-1. Chin J Nat Med, 2019. 17(3): p. 198-208.

144. Marchese, S., et al., Aflatoxin B1 and M1: Biological Properties and Their Involvement in Cancer Development. Toxins (Basel), 2018. 10(6).

145. Guerra, A.R., M.F. Duarte, and I.F. Duarte, Targeting Tumor Metabolism with Plant-Derived Natural Products: Emerging Trends in Cancer Therapy. J Agric Food Chem, 2018. 66(41): p. 10663-10685.

146. Kim, C. and B. Kim, Anti-Cancer Natural Products and Their Bioactive Compounds Inducing ER Stress-Mediated Apoptosis: A Review. Nutrients, 2018. 10(8).

147. Bayir, A.G., H.S. Kiziltan, and A. Kocyigit, Chapter 1 - Plant Family, Carvacrol, and Putative Protection in Gastric Cancer, in Dietary Interventions in Gastrointestinal Diseases, R.R. Watson and V.R. Preedy, Editors. 2019, Academic Press. p. 3-18.

148. Roe, A.L., et al., Assessing Natural Product-Drug Interactions: An End-to-End Safety Framework. Regulatory Toxicology and Pharmacology, 2016. 76: p. 1-6.

149. Jia, J., et al., Mechanisms of drug combinations: interaction and network perspectives. Nat Rev Drug Discov, 2009. 8(2): p. 111-28.

150. Kim, H.U., et al., A systems approach to traditional oriental medicine. Nat Biotechnol, 2015. 33(3): p. 264-8. 